\title{
Sonic hedgehog accelerates DNA replication to cause replication stress promoting cancer initiation in medulloblastoma
}

${ }^{1}$ Montreal Clinical Research Institute (IRCM), 110 Pine Ave West, Montreal, Quebec, Canada, H2W 1R7

${ }^{2}$ Integrated Program in Neuroscience, McGill University, Montreal, Quebec, Canada, H3A 2B4

${ }^{3}$ MRC Human Genetics Unit, MRC Institute of Genetics and Molecular Medicine, University of Edinburgh, Edinburgh EH4 2XU, UK

${ }^{4}$ Department of Biochemistry and Donnelly Centre for Cellular and Biomolecular Research. University of Toronto. Toronto, Ontario, Canada, M5S 3E1

${ }^{5}$ Institute for Research in Immunology and Cancer (IRIC). University of Montreal, Montreal, Quebec, Canada, H3T 1J4

${ }^{6}$ Department of Anatomy and Cell Biology, Division of Experimental Medicine, McGill University, Quebec, Canada, H3A 2B2

${ }^{7}$ Department of Medicine, University of Montreal, Montreal, Quebec, Canada, H3T 1J4

\# Current affiliation: MRC Human Genetics Unit, MRC Institute of Genetics and Molecular Medicine, University of Edinburgh.

$\&$ These authors contributed equally to this work.

* Correspondence to: Frédéric Charron, Ph.D.

Molecular Biology of Neural Development

Montreal Clinical Research Institute (IRCM)

110 Pine Ave West, Montreal, Quebec, Canada, H2W 1R7

+1 5149875773 (telephone) +15149875774 (fax)

Frederic.Charron@ircm.qc.ca 


\section{Summary}

The mechanisms generating cancer-initiating mutations are not well understood. Sonic hedgehog (SHH) pathway activation is frequent in medulloblastoma (MB), with PTCH1 mutations being a 
61 Granule cell progenitors (GCPs) of the cerebellum produce the largest neuronal population in the human brain ${ }^{1}$ and proliferate in response to Sonic hedgehog $(\mathrm{Shh})^{2}$. GCPs are also the cell of origin of $\mathrm{SHH}-\mathrm{MB}^{3-5}$, a common subtype of the most frequent malignant pediatric brain tumor ${ }^{6}$, most commonly caused by Shh pathway mutations ${ }^{7}$. Mutations in the SHH receptor PTCH1 are frequent genetic drivers of $\mathrm{SHH}-\mathrm{MB}^{8}$, and $\mathrm{PTCH} 1$ is also mutated Gorlin syndrome, a cancer predisposition disorder. PTCH1 is a tumor suppressor and acts to negatively regulate intracellular downstream SHH signaling. Using MB-prone $P t c h 1^{+/-}$mice, we previously found that loss of heterozygosity ( $\mathrm{LOH}$ ) of the wild-type Ptch1 allele is the molecular event leading to preneoplasia formation and MB initiation ${ }^{9}$; however, the cause and molecular mechanism leading to PTCH1 LOH and MB remained to be defined.

It has been proposed that oncogene or aberrant growth factor activation in precancerous lesions induces replication stress and DNA damage ${ }^{10,11}$, fueling genomic instability and cancer growth ${ }^{12}$. While a link between oncogene activation and malignant growth has been established in many cancer types, little is known about the molecular causes leading to the acquisition of tumorinitiating mutations in normal tissues, an outstanding problem in the cancer field ${ }^{13}$. Working on a unique system that allowed us to study GCPs in vivo and ex vivo, we tested whether the normal proliferative effects of the developmental morphogen Shh can lead to genomic instability and DNA lesions responsible for tumor initiation in the SHH-MB cell-of-origin. DNA replication stress and somatic recombination in neural progenitor cells. In contrast to 
oncogene-induced replication stress ${ }^{14,15}$, we found that Shh-induced replication stress does not lead to replication inhibition but is instead associated with a concomitant increase in both replication origin firing and fork speed. Enhanced Shh-dependent origin firing is mediated by helicase loading and activation, that promotes replication stress and recombination. The increase in replication origins in Shh-exposed GCPs is dependent on Cdc7, a kinase regulating origin firing, and reducing Shh-dependent origin firing via Cdc7 inhibition blocks Shh-replication stress and MB initiation in tumor-prone mice.

\section{Results}

\section{Large-scale deletions and recombination events cause PTCH1 LOH in medulloblastoma}

To investigate the mechanism of tumor initiation in SHH-MB, we first determined the nature of the mutations that cause biallelic PTCH1 inactivation in human and mice. Most mutations in the first PTCH1 allele in human SHH-MBs are indels and SNVs. Reanalysis of a human MB cohort ${ }^{8}$ indicated that $77 \%(43 / 56)$ of those cases additionally display loss of the wild-type allele as a result of 9q deletions (29/56; 52\%; copy loss-LOH) or 9q copy-neutral LOH (14/56; 25\%) (Fig. 1a,b). Copy-neutral LOH (CN-LOH) involves the duplication of one chromosome segment or whole chromosome along with the loss of the corresponding homologous region, and can be due to chromosome segregation errors or recombination events ${ }^{16}$. Thus, we further analyzed CNLOH events in human SHH-MB to identify the cause of CN-LOH. All cases displayed large regions of homozygosity (9q CN-LOH) comprising and neighboring the PTCH1 locus, while the centromere and $9 \mathrm{p}$ arm of the chromosome retained heterozygosity, indicating that the $\mathrm{CN}-\mathrm{LOH}$ event responsible for the loss of the PTCH1 wild-type allele is homologous recombination (Fig. 1a). 
As well, in advanced Ptch1 ${ }^{+/-}$MBs in mice, we identified megabase-long segments of homozygosity on chromosome 13 (Fig. 1c) causing CN-LOH, consistent with previous reports ${ }^{17,18}$. Since the chromosome containing the first Ptch1 mutant allele is used as recombination template, these changes result in biallelic loss of Ptch1, evidenced as complete absence of Ptch1 exon 1-2, the genomic region corresponding to the engineered Ptch1 mutation ${ }^{19}$ (Fig. 1e). Consistent with CN-LOH, whole chromosome 13 arm losses or big deletions were not detected, as assessed by comparative genome hybridization arrays and chromosome paints (Fig. 1d-f). These findings indicate that the mutation events leading to Ptch1 LOH in most (9/11; $82 \%)$ Ptch $^{+/-}$MBs and at least $25 \%$ of human MBs are somatic (mitotic) recombination events, which result in copy-neutral LOH (Fig. 1a).

\section{Sonic hedgehog causes DNA replication stress}

DNA replication stress, a state of deregulated DNA replication ${ }^{20}$, is a major cause of LOH and $\mathrm{CNVs}^{21,22}$. During the early stages of cancer development, oncogene-induced replication stress (OI-RS) causes DNA breaks and genomic instability. This in turn promotes multistage carcinogenesis by driving extensive $\mathrm{LOH}$ and inactivation of tumor suppressor genes ${ }^{10,11,23}$. Since Shh is a well-established mitogen for GCPs ${ }^{2}$, we used primary GCPs to test whether Shh causes DNA replication stress in the SHH-MB cell-of-origin. This is a plausible hypothesis in light of our previous observation that Shh induces $\gamma$-H2AX and DNA breaks in GCPs ${ }^{24}$, and based on previous studies showing that neuronal progenitors, including GCPs, require the presence of essential S-phase checkpoint proteins to maintain genomic stability ${ }^{25-27}$. 
We investigated whether physiological levels of Shh cause DNA replication stress during Sphase by performing short BrdU pulses in wild-type GCPs cultured in the absence or presence of Shh. Consistent with Shh inducing replication stress in S-phase, enhanced $\gamma-\mathrm{H} 2 \mathrm{AX}$ levels were seen in Shh-treated GCPs specifically in S-phase (Fig. 2a, Extended Data Fig. 1a). $\gamma$-H2AX foci

Although Shh is the most potent mitogen for GCPs, other growth factors such as Egf or Igf1 also induce GCP proliferation ${ }^{2}$. To investigate whether GCP proliferation alone is sufficient to induce replication stress foci, we stimulated GCPs with Igfl or Shh. Interestingly, while $100 \mathrm{ng} / \mathrm{ml}$ Igf1 leads to similar proliferation levels as $10 \mathrm{nM}$ Shh (Fig. 2e,f; Extended Data Fig. 1i), only Shh but not Igf1, induces $\gamma$-H2AX (Fig. 2g), indicating that proliferation itself is not sufficient to induce replication stress foci. This effect of Shh (compared to Igf1 and $\mathrm{Ctl}$ ) was present even when $\gamma$ - 
H2AX levels were measured only in S-phase GCPs (Extended Data Fig. 1k), indicating that it is not due to difference in the ability of the mitogens to drive proliferation. Additionally, other (weaker) GCP mitogens (bFgf and Egf) did not induce $\gamma$-H2AX in GCPs (Extended Data Fig. $1 \mathrm{i}, \mathrm{j}$ ), further suggesting that replication stress is specific to Shh. Moreover, GCPs proliferating in response to Shh in vivo in the EGL display more $\gamma$-H2AX than other highly proliferative tissues such as the intestinal epithelium (Extended Data Fig. 2a-d). Together, these results indicate that enhanced replication stress was not the result of proliferation per se but the specific consequence of Shh-dependent DNA replication.

\section{Increased fork speed and origin firing distinguish Shh-induced replication stress}

DNA replication stress is defined as any condition associated with accumulation of singlestranded DNA (ssDNA) or slowing or stalling of replication fork progression ${ }^{20}$. Shh-treated GCPs display high levels of ssDNA in S-phase (chromatin-bound Rpa32, Fig. 2h), indicative of replication stress. To investigate how Shh might cause replication stress, we performed DNA combing in GCPs directly isolated from mouse cerebella and cultured ex-vivo for 24 h. (Fig. 3a). Shh did not cause replication fork asymmetry or stalling as assessed by DNA combing (Fig. 3b,e). In contrast, Shh, but not Igfl, led to a marked, $40 \%$ increase in fork speed (Fig. 3c,e). Additionally, Shh, but not Igf1, caused a 7.4-fold increase in active fork density, a proxy of replication origin firing (Fig. 3d; see also Extended Data Fig. 4a and Fig 5c-g). This effect is not simply the consequence of proliferation since Igfl, like Shh, induced a 2-fold increase in the fraction of BrdU-positive cells but did not enhance origin firing (Fig. 2f). Consistent with these effects on DNA replication, Shh accelerated S-phase progression to reduce the length of S-phase (Fig. 3f,g). Since these effects on DNA replication are qualitatively different from the classical 
definition of DNA replication stress, we term this Shh-induced replication stress (Shh-RS) (Fig. 3h,i). As far as we know, that Shh increases both origin firing and fork speed is a unique feature; indeed, this seems to be an exception to the general rule that origin firing and fork speed are anticorrelated $^{28-30}$. Another feature distinguishing Shh-RS from classical and oncogene-induced RS is the presence of 53bp1 foci in S-phase and not in G1 (Fig. 2c).

We performed RNA sequencing to explore possible mechanisms governing Shh-dependent changes on DNA replication (Extended Data Fig. 2e). Shh induces expression of essential nucleotide metabolism genes (Extended Data Fig. 3a,b), and mass spectrometry revealed Shhdependent increases in NTP levels (Extended Data Fig. 3c), suggesting the possibility that Shh increases dNTP levels to sustain a high fork speed, leading to DNA damage. If this is the case, nucleotide supplementation in absence of Shh should lead to high DNA fork rates and increased $\gamma$-H2AX. Notably, nucleosides increased $\gamma$-H2AX and accelerated S-phase in absence of Shh, but did not augment DNA fork speed (Extended Data Fig. 3f-h). We found that nucleoside supplementation was instead associated with a 2.1-fold increase in origin firing (Extended Data Fig. 3i), suggesting that increased origin firing, rather than fork speed, could be responsible for Shh-induced replication stress.

\section{Shh promotes Mcm loading at replication origins}

Shh resulted in a larger ( 7.4 fold) increase in origin firing compared to nucleoside supplementation (Extended Data Fig. 4a), suggesting additional mechanisms mediating replication initiation by Shh. DNA replication is initiated by the binding of origin recognition protein complexes (Orc) to DNA sequences called replication origins ${ }^{31}$. This event is followed 
by binding of the licensing factor Cdc6, which subsequently recruits Cdt1 and the Mcm2-7 heterohexameric complex onto chromatin ${ }^{32}$. This pre-replication complex (pre-RC) loading onto chromatin during G1 is called origin licensing. Origin activation requires the association of

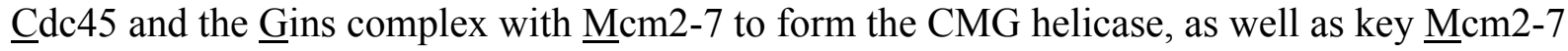
phosphorylation events catalyzed by $\mathrm{Cdc} 7^{33,34}$ for origin firing in S-phase. Our gene expression studies revealed that Shh induces expression of pre-replicative complex, licensing factors, and

DNA helicase genes (Fig. 4a, Extended Data Fig. 4b-d). To investigate whether Shh regulates origin licensing, we tested whether Shh also promotes loading of Mcm2-7 onto chromatin. In asynchronous GCPs, Shh increased nuclear and chromatin-bound Mcm2 (Fig. 4b,c,e); other Mcm subunits like Mcm7 are also recruited to chromatin in response to Shh (Fig. 4d), a result expected from the fact that Mcm2-7 is recruited as a full hexamer and indicates that chromatinbound Mcm2 is a good readout of origin licensing in our system. Consistent with general DNA replication principles ${ }^{31}$, Shh-dependent origin licensing happens during G1, and Shh-treated GCPs enter S-phase with higher chromatin-bound Mcm2 (Fig. 4f-h, and Extended Data Fig. 4fg). However, pre-RCs are removed from chromatin as cells progress through S-phase, and there is no difference in chromatin-bound $\mathrm{Mcm} 2$ between $\mathrm{Ctl}$ and Shh conditions in late S-phase (Fig. $4 \mathrm{~g}$ ), indicating that Shh does not cause re-licensing in S-phase. Altogether, these results demonstrate that Shh promotes replication origin licensing (Fig. 4i), that could contribute to increased origin density during S-phase.

\section{Shh induces additional origin firing in a Cdc7-dependent manner}

Many origins usually remain dormant during unperturbed S-phase progression; therefore, our expectation was that increased origin activation would also occur, to account for increased 
replication fork density. Hence, we tested if Shh also promotes helicase activation. Shh treatment of GCPs induced expression of the key helicase activators Cdk2, Dbf4 and Cdc7 (Extended Data

Fig. 4e) and led to Mcm2 phosphorylation in a Cdc7-dependent manner (Fig. 5a and Extended Data Fig. 5a), the critical event leading to helicase activation ${ }^{33}$. Shh also induced chromatin recruitment of Gins2 in a Cdc7-dependent manner, another indicator of helicase activation (Extended Data Fig. 6b).

We next confirmed that these markers of helicase activation did indeed correspond to increased number of active origins. First, we addressed whether the change in proportion of S-phase cells could be a confounding factor for fork density measurements. However, normalization to Sphase fraction to control for proliferation rate still demonstrated that Shh increases fork density (Fig. 5c-e). To confirm that increased origin density was directly associated with Mcm2 phosphorylation, we again assessed fork density in GCPs and found that Cdc7i blocked Shhdependent increase in p-Mcm2 levels and elevation in origin density (Fig. 5a-e). This was seen at low doses of Cdc7i, that did not affect S-phase fraction, fork speed (Extended Data Fig. 7a,b), or baseline Mcm2 phosphorylation.

Furthermore, to corroborate this finding, we measured inter-origin distances (IODs) by DNA combing in GCPs. Shorter IODs reflect increased origin density and are the gold-standard to evaluate origin activation. In keeping with the fork density measurements, we observed that Shh led to a substantial reduction in IODs (Control: 132kb; Shh: 73kb; Fig 5f,g). Similarly, Cdc7i blocked Shh dependent reduction in IODs, a result supported by a different Cdc7 inhibitor (Extended Data Fig. 6c).

Additionally, we reasoned that reducing Mcm2 levels should also limit the ability of Shh to induce origin firing. We therefore used GCPs isolated from Mcm2 hypomorphic mice (here 
termed $\mathrm{Mcm}^{+/-}$) in which $\mathrm{Mcm} 2$ levels are reduced to $60 \%$ of normal ${ }^{35}$. Consistent with this prediction, Shh markedly reduced IODs in $\mathrm{Mcm}^{+/+}$wildtype GCPs, and this Shh-dependent as a central event in Shh-induced replication initiation. induction in origin firing was attenuated in $\mathrm{Mcm}^{+/-}$GCPs (Fig. 5h). Together, our findings suggested a model in which Shh-enhanced origin firing was the result of both Mcm loading and origin licensing, and increased Cdc7 activation of origins (Fig. 5i). Hence, these experiments demonstrated that Shh induces origin firing, implicating the Cdc7-dependent activation of Mcm2

\section{EdU-seq in GCPs reveals Shh-dependent replication initiation domains}

The increase in activated origins in Shh-treated GCPs would predict additional genomic regions being coopted as replication origins. Therefore, as an orthogonal method to single molecule analyses, we mapped replication initiation zones genome-wide using EdU-seq ${ }^{36,37}$. As expected, we identified common initiation zones present in both Ctl- and Shh-treated GCPs (Fig. 6a). Consistent with the DNA combing experiments, Shh-treated GCPs displayed higher numbers of nascent DNA regions, which we term Shh-dependent initiation zones (Fig. 6a). In agreement with a recent report ${ }^{37}$, EdU peaks are enriched at poly A-T tracts and other repetitive sequences, with most firing events in GCPs mapping to intergenic (70\%) and intronic (27\%) regions (Extended Data Fig. 6d-f). Using replication timing data (Repli-seq ${ }^{38}$ ), we found that Shh-treated GCPs display a higher proportion of Edu-seq peaks in early replicating regions (Fig. 6b). Also, among the $150 \mathrm{CNV}$ regions identified in Ptch $1^{+-}$MBs, 22 (13\%) matched replication initiation regions in Ctl-treated GCPs, while 50 (33\%) of them matched origins in Shh-treated GCPs (Fig. 6c), suggesting a correlation between origin firing and genome structural changes in MB, noteworthy as firing regions have been reported to be prone to breakage in mammalian cells ${ }^{37}$. 
Consistent with Cdc7 effects mediating Shh-dependent replication initiation, we also observed using EdU-seq that Cdc7-inhibition attenuated Shh-dependent replication initiation (Fig. 6d and

Extended Data Fig. 6g). (Note that, as EdU-seq only maps a subset of origins/replication initiation zones $^{39}$, the distances between initiation zones are larger than IODs measured by combing.) In summary, the EdU-seq analysis demonstrated the recruitment of additional genomic regions as sites of replication initiation in response to Shh.

\section{Shh-dependent origin firing is required for Shh-dependent replication stress}

To establish whether Shh-dependent origin firing is required for Shh-induced replication stress, we used Cdc7i at low doses that do not perturb cell cycle progression while blocking Shhdependent origin firing (Fig. 5 and Extended Data Fig .6a). Under these conditions, Cdc7i blocked Shh-induced $\gamma$-H2AX and ssDNA accumulation (Fig. 7 a-d), consistent with increased origin firing promoting DNA replication stress, establishing that increased origin firing is upstream of Shh-dependent replication stress and not a consequence of it. Notably, this differs from many instances of OI-RS where increased origin firing is a response to replication fork stalling caused by oncogenes ${ }^{14,15}$ (Fig. 3).

\section{Shh induces recombination in a Cdc7-dependent manner}

We next investigated the consequences of Shh-induced replication stress for genomic instability and specifically how genomic changes responsible for PTCH1 LOH in mouse and human SHHMBs might occur. Since copy-neutral LOH events leading to PTCH1 loss are the result of somatic homologous recombination (Fig. 1a), we assessed whether Shh-dependent replication stress leads to hyper-recombination. Supporting this hypothesis, Shh increases the expression of 
essential homologous recombination (HR) genes but not non-homologous end-joining (NHEJ)

291 genes (Extended Data Fig. 7a-d). We observed that Shh increased total and chromatin-bound

292 Rad51 levels (Extended Data Fig. 7e,f), a key ssDNA binding factor in HR, in S-phase GCPs, 293 while 53bp1 foci (which promote NHEJ) were not increased (Extended Data Fig. 7g). This 294 suggested that ssDNA generated during Shh-induced replication stress in GCPs could be the 295 source of homology-directed repair events. Furthermore, using the sister chromatid exchange 296 (SCE) assay, a technique to assess the frequency of DNA recombination events, we found that 297 Shh promotes hyper-recombination in a Cdc7-dependent manner (Fig. 7e,f); in contrast, other 298 GCP mitogens did not induce SCEs (Fig. 7e and Extended Data Fig. 7h). Moreover, increased 299 HR was also evident using RaDR-GFP mice, which contain a truncated GFP reporter gene to 300 detect spontaneous recombination events originating from DNA breaks or broken replication 301 forks ${ }^{40}$, confirming that Shh-RS is associated with increased recombination (Extended Data Fig. 302 7i). We conclude that Shh-induced replication stress promotes HR events that could enhance 303 LOH in GCPs, implicating the developmental mitogen Shh as a cause of hyper-recombination.

\section{DNA replication and recombination signatures in SHH-MB- $\alpha$}

As we implicated $\mathrm{Cdc} 7$ as a mediator of Shh-dependent genomic instability, we next assessed the relevance of this finding to clinical outcome in human medulloblastoma. In keeping with our mouse model, high CDC7 expression is an indicator of poor prognosis $\left(p=2.4 \mathrm{e}^{-4}\right)$ across all four medulloblastoma groups (WNT, SHH, Group 3 and Group 4) and within SHH-MB (p=0.044) (Extended Data Fig. 8a,b). The four MB groups have recently been divided in 12 subtypes, where SHH-MBs have been categorized in 4 subtypes (SHH-MB- $\alpha, \beta, \gamma, \delta)^{41}$. SHH-MB- $\alpha$ is the subtype with worst prognosis, TP53 mutations, and the highest frequency of broad CNV 
313 (including chromosome $9 \mathrm{q}$ alterations containing PTCH1) ${ }^{41}$. It is remarkable that Ptch $1^{+/-}$mice 314 also acquire Ptch1 LOH and spontaneous p53 mutations ${ }^{9,42}$ and therefore constitute a model for 315 SHH-MB- $\alpha$. Furthermore, we found that SHH-MB- $\alpha$ display high levels of CDC7 and a 316 distinctive DNA replication and homologous recombination gene ontology signature (Extended 317 Data Fig. 8c-f) ${ }^{41}$, resembling the effects of Shh on GCPs. Recent proteomics-based classification 318 of MB identified 2 subtypes of SHH-MBs (SHHa and $\mathrm{SHHb})^{43}$ wherein SHHa displays DNA 319 replication and DNA recombination proteomic and gene expression signatures compared to 320 SHHb (Extended Data Fig. 9a-b). Importantly, SHHa displays high MCM2 mRNA and protein 321 levels, and the key CDC7-dependent phosphorylation events leading to MCM2 activation (p322 Ser40, p-Ser139, and p-Ser26/27) are also upregulated in human SHHa (Extended Data Fig. 9c$323 \mathrm{e}$ ). These phenotypes in subsets of human SHH-MB mirror the gene expression and phenotypic 324 effects induced by Shh in GCPs, suggesting direct relevance of our findings on Shh-replication 325 stress arising from origin licensing and activation. Thus, we propose that changes in gene expression driven by Shh in GCPs generate DNA replication stress, hyper-recombination and MB-causing mutations characterizing both human SHH-MB- $\alpha /$ a and murine Ptch1 MB.

\section{in vivo Cdc7 inhibition prevents $\mathrm{MB}$ initiation in Ptch1 $^{+/-}$mice}

Finally, we wished to establish the relevance of our findings to cancer-initiation in MB. Because Cdc7i inhibition blocks Shh-dependent replication stress and recombination, we predicted that in vivo Cdc7 inhibition should decrease the likelihood of Ptch1 LOH events. Since Ptch1 LOH is the MB-initiating event preceding preneoplasia ${ }^{9}$, we hypothesized that in vivo Cdc7 inhibition during Shh-dependent GCP proliferation, when GCPs are at risk of Ptch1 LOH, should decrease preneoplasia incidence. We therefore treated tumor-prone $P t c h 1^{+/-}$mice from postnatal day 1 
(P1) to P16 with $2 \mathrm{mg} / \mathrm{kg} /$ day TAK-931, a validated Cdc7 inhibitor (Cdc7i) used in clinical trials ${ }^{44}$, or vehicle (Fig. 8a). We used low Cdc7i doses (20 times lower than the ones used in vivo to block the growth of other tumor types ${ }^{44}$ so as not to block cell proliferation while reducing the level of helicase activation. Under these conditions, Cdc7i did not affect mouse growth and at P7 had no effect on EGL thickness, a direct correlate of total GCP number (Fig 8b,c and Extended Data Fig. 10a-e). As well, GCP proliferation in P7 cerebella was unaltered, as measured using three proliferation markers (Ki67, phospho-histone H3 and BrdU; Extended Data Fig. 10f-i). Cdc7i did not alter cerebellar development since cerebellum mid-sagittal area and IGL area and perimeter were normal at P16 (Extended Data Fig. 10c,d), further supporting Cdc7i at this dose not affecting GCP proliferation. Furthermore, Cdc7i did not affect cerebellar balance function as assessed by the rotarod test (Fig. 8e).

In contrast, using in vivo DNA combing, we found that Cdc7i reduced origin firing in vivo (Fig. 8i). Also as expected from in vitro results (Fig. 5a), Cdc7i reduced helicase activation (p-Mcm2), and the rate of DNA synthesis (BrdU fluorescence intensity/cell) (Fig. 8f-h and Extended Data Fig. 10j,k), but not the number of BrdU+ cells (Extended Data Fig. 10h). Strikingly, Cdc7i also reduced recombination rate in vivo (Fig. 8j), and Cdc7 inhibition for 2 weeks led to a five-fold reduction in preneoplasia incidence. This was reduced from $63 \%(9 / 14)$ to $12.5 \%(2 / 16)$, and the number of preneoplastic lesions per cerebellum was decreased compared to vehicle-treated animals (Figure 8k-m). These results therefore supported our model that reduction of Shhdependent DNA replication stress in tumor-prone Ptch1 ${ }^{+/}$GCPs before cancer initiation is capable of preventing medulloblastoma-initiating mutations. 
Recent work has shown that mutations resulting from cell division account for two thirds of the mutations in human cancers ${ }^{13}$, indicating that DNA replication errors are a major source of cancer-causing mutations. Therefore, investigating ways to render DNA replication 'safer' in tumor-initiating cells becomes an attractive aim for cancer biology ${ }^{13}$. However, current knowledge on DNA replication in metazoans is primarily based on studies performed using Xenopus egg extracts, drosophila embryos and immortalized cell lines ${ }^{45}$, none of which is the cell of origin of cancer. Although several studies have determined how oncogenic signaling leads to chromosomal instability at precancerous stages or in cell lines expressing oncogenes ${ }^{23,46}$, fewer have investigated how oncogenic signaling leads to genomic instability in primary cells, and many of them are restricted to hematopoietic stem cells ${ }^{47}$. Moreover, very few studies have investigated how DNA replication-associated processes affect the function of primary or stem cells $^{48}$, and little is known about how DNA replication stress causes genomic instability in primary cell populations at risk of transformation.

Here we show that Shh, a developmental mitogen, alters DNA replication dynamics in GCPs, the SHH-MB cells of origin. This leads to DNA replication stress and elevated homologous recombination. Although Shh caused ssDNA accumulation, it did not cause fork stalling or asymmetry, establishing that previously demonstrated Shh-induced DNA breaks ${ }^{24}$ are not the consequence of replication inhibition. Indeed surprisingly, we discovered that Shh in fact induces both increased replication fork speed and origin firing. Several classical oncogenes are known to induce origin firing ${ }^{15}$, however depleting replication substrates and leading to fork slowing/stalling ${ }^{49}{ }^{15}$. Shh-RS is associated with faster DNA replication and shortened S-phase, in contrast to OI-RS where S-phase checkpoint activation, S-phase lengthening and arrest may 
occur $^{50}$. As well, unlike OI-RS, Shh-RS is not associated with the presence of persisting replication intermediates such as ultrafine anaphase bridges or 53bp1 foci in G1 (which are the outcome of replication inhibition after mitosis). Therefore Shh-RS and OI-RS appear qualitatively different (Fig. 3h,i).

There is a well-documented negative correlation between fork rate and origin firing ${ }^{28,29}$, also observed in the RS phenotype caused by PARP inhibition ${ }^{51}$. This correlation is likely the result of limited availability of DNA substrates, such as dNTPs and replication factors ${ }^{28,29,52-55}$. Therefore, cells adjust the number of active origins in accordance to fork speed to complete Sphase in a timely fashion ${ }^{30,56}$. Hence, for Shh to cause such a marked reduction in S-phase time, a simultaneous increase in origin firing and fork speed is necessary. The strong transcriptional effects of Shh increasing nucleotide metabolism and replication proteins could ensure this Sphase acceleration. That GCPs physiologically exposed to Shh coopt flexible or dormant origins to achieve such fast replication is also intriguing. We propose that Shh-induced replication stress is a trade-off of this Shh-dependent acceleration of S-phase required for the rapid production of the largest neuronal population in the brain.

As both Shh-RS and Shh-induced origin firing are blocked by Cdc7i, we favor a model where increased origin firing is due to Shh increasing Cdc7 activity, with elevated origin firing then being a major source of Shh-RS (Fig. 8i). Supporting this notion, increased Mcm2phosphorylation is observed, with the Mcm helicase and Cdc45 being the best characterized Cdc7 substrates, and a major function of $\mathrm{Cdc} 7$ being regulation of origin firing ${ }^{57}$. Furthermore, in keeping with this model, Shh-origin activation was dependent on availability of Mcms, being 
attenuated in $\mathrm{Mcm}^{+/-}$GCPs. However, Cdc7 phosphorylates other substrates in other cellular processes $^{57,58}$; thus, other Cdc7 targets may also contribute to Shh-RS. As well, increased fork velocity was independent of $\mathrm{Cdc} 7$, suggesting that additional pathways may also contribute to Shh-RS.

Shh-dependent replication stress is associated with elevated homologous recombination. This could arise from accelerated S-phase and high density of replication forks. This would reduce the time available to deal with replication of difficult genomic regions, and/or repair of endogenous DNA lesions, that are then instead dealt with by increased homologous recombination events (Fig. 8i). Such increased somatic recombination provides a mechanism that will promote LOH in GCPs, the cell of origin of Shh-MB, particularly CN-LOH events. We were able to test this possibility in a preclinical MB model, given the dependency of Shh-RS on Cdc7 activity, harnessing the availability of a well characterized Cdc7 inhibitor. This resulted in reduced Shhinduced somatic recombination, alongside decreased preneoplastic cerebellar lesions. This therefore provides a 'proof of principle' demonstrating that attenuating DNA-replication-stress in primary cells at risk of transformation abrogates tumor-initiation. This work also extends the developmental functions of $\mathrm{Shh}^{59,60}$, to consider its consequence as a developmental mitogen on DNA replication and genome stability and suggests an approach that may be applicable in some clinical contexts to render DNA replication potentially safer to prevent cancer initiation.

Acknowledgments: We thank A. Helness, S. Terouz, E. Massicotte, F. Depault, J. Cardin, and J. Barthe for expert assistance; A. Dumont and M. Rondeau for RNAseq library preparation and O. Neyret for EdU-seq library preparation. We thank O. Jumanca and the IRCM animal facility staff 
for animal handling. We thank E. Drobetsky for critical reading of the manuscript. We thank the International Cancer Genome Consortium (ICGC) for providing access to human MB data. L.TO. was supported by the Caldas (Colciencias) and the Djavad Mowafaghian Foundation fellowships and is now supported by an EMBO-LTF (ALTF-739-2019). D.G. was supported by a post-graduate scholarship from the Natural Sciences and Engineering Research Council of Canada. Funding: This work was supported by the Canadian Institutes of Health Research (FDN334023 to F.C.), the Fonds de Recherche du Québec-Santé (to F.C.), the Canada Foundation for Innovation (33768 to F.C.), the Canadian Cancer Society Research Institute (Impact grant 702310 to G.W.B.), the Medical Research Council, UK (MRC, MC_UU_00007/5 to A.P.J.), and the European Union's Horizon 2020 research and innovation programme ERC Advanced Grant (no. 788093 to A.P.J.). F.C. holds the Canada Research Chair in Developmental Neurobiology.

Author contributions: L.T-O. and F.C. conceived the study. L.T-O., D.G., F.R., S.M., S.S and B.H. performed experiments. A.B. performed in silico analyses. T.H. provided reagents and advice. D.G. and L.T-O. analyzed data. A.P.J. provided critical advice on manuscript and supported parts of the work, as well as the first author during revision. L.T-O., G.W.B, A.P.J., and F.C. wrote the manuscript. Competing interests: Authors declare no competing interests. 
1 Azevedo, F. A. et al. Equal numbers of neuronal and nonneuronal cells make the human brain an isometrically scaled-up primate brain. The Journal of comparative neurology 513, 532-541, doi:10.1002/cne.21974 (2009).

2 Wechsler-Reya, R. J. \& Scott, M. P. Control of neuronal precursor proliferation in the cerebellum by Sonic Hedgehog. Neuron 22, 103-114 (1999).

3 Oliver, T. G. et al. Loss of patched and disruption of granule cell development in a preneoplastic stage of medulloblastoma. Development 132, 2425-2439, doi:10.1242/dev.01793 (2005).

4 Yang, Z. J. et al. Medulloblastoma can be initiated by deletion of Patched in lineagerestricted progenitors or stem cells. Cancer cell 14, 135-145, doi:10.1016/j.ccr.2008.07.003 (2008).

5 Schuller, U. et al. Acquisition of granule neuron precursor identity is a critical determinant of progenitor cell competence to form Shh-induced medulloblastoma. Cancer cell 14, 123-134, doi:10.1016/j.ccr.2008.07.005 (2008).

6 Kool, M. et al. Molecular subgroups of medulloblastoma: an international meta-analysis of transcriptome, genetic aberrations, and clinical data of WNT, SHH, Group 3, and Group 4 medulloblastomas. Acta neuropathologica 123, 473-484, doi:10.1007/s00401012-0958-8 (2012).

7 Northcott, P. A. et al. Medulloblastomics: the end of the beginning. Nature reviews. Cancer 12, 818-834, doi:10.1038/nrc3410 (2012).

8 Northcott, P. A. et al. The whole-genome landscape of medulloblastoma subtypes. Nature 547, 311-317, doi:10.1038/nature22973 (2017).

9 Tamayo-Orrego, L. et al. Evasion of Cell Senescence Leads to Medulloblastoma Progression. Cell reports 14, 2925-2937, doi:10.1016/j.celrep.2016.02.061 (2016).

10 Bartkova, J. et al. DNA damage response as a candidate anti-cancer barrier in early human tumorigenesis. Nature 434, 864-870, doi:10.1038/nature03482 (2005).

11 Gorgoulis, V. G. et al. Activation of the DNA damage checkpoint and genomic instability in human precancerous lesions. Nature 434, 907-913, doi:10.1038/nature03485 (2005).

12 Negrini, S., Gorgoulis, V. G. \& Halazonetis, T. D. Genomic instability--an evolving hallmark of cancer. Nature reviews. Molecular cell biology 11, 220-228, doi:10.1038/nrm2858 (2010).

13 Tomasetti, C., Li, L. \& Vogelstein, B. Stem cell divisions, somatic mutations, cancer etiology, and cancer prevention. Science 355, 1330-1334, doi:10.1126/science.aaf9011 (2017).

14 Macheret, M. \& Halazonetis, T. D. DNA replication stress as a hallmark of cancer. Annual review of pathology 10, 425-448, doi:10.1146/annurev-pathol-012414-040424 (2015).

15 Hills, S. A. \& Diffley, J. F. DNA replication and oncogene-induced replicative stress. Current biology : CB 24, R435-444, doi:10.1016/j.cub.2014.04.012 (2014).

16 Lasko, D., Cavenee, W. \& Nordenskjold, M. Loss of constitutional heterozygosity in human cancer. Annu Rev Genet 25, 281-314, doi:10.1146/annurev.ge.25.120191.001433 (1991). 
17 Pazzaglia, S. et al. Two-hit model for progression of medulloblastoma preneoplasia in Patched heterozygous mice. Oncogene 25, 5575-5580, doi:10.1038/sj.onc.1209544 (2006).

18 Ishida, Y. et al. Genomic and gene expression signatures of radiation in medulloblastomas after low-dose irradiation in Ptch1 heterozygous mice. Carcinogenesis 31, 1694-1701, doi:10.1093/carcin/bgq145 (2010).

19 Goodrich, L. V., Milenkovic, L., Higgins, K. M. \& Scott, M. P. Altered neural cell fates and medulloblastoma in mouse patched mutants. Science 277, 1109-1113 (1997).

20 Zeman, M. K. \& Cimprich, K. A. Causes and consequences of replication stress. Nature cell biology 16, 2-9, doi:10.1038/ncb2897 (2014).

21 Zheng, D. Q., Zhang, K., Wu, X. C., Mieczkowski, P. A. \& Petes, T. D. Global analysis of genomic instability caused by DNA replication stress in Saccharomyces cerevisiae. Proceedings of the National Academy of Sciences of the United States of America 113, E8114-E8121, doi:10.1073/pnas.1618129113 (2016).

22 Arlt, M. F. et al. Replication stress induces genome-wide copy number changes in human cells that resemble polymorphic and pathogenic variants. Am J Hum Genet 84, 339-350, doi:10.1016/j.ajhg.2009.01.024 (2009).

23 Di Micco, R. et al. Oncogene-induced senescence is a DNA damage response triggered by DNA hyper-replication. Nature 444, 638-642, doi:10.1038/nature05327 (2006).

24 Mille, F. et al. The Shh receptor Boc promotes progression of early medulloblastoma to advanced tumors. Developmental cell 31, 34-47, doi:10.1016/j.devcel.2014.08.010 (2014).

25 Lee, Y. et al. ATR maintains select progenitors during nervous system development. The EMBO journal 31, 1177-1189, doi:10.1038/emboj.2011.493 (2012).

26 Lee, Y. et al. Neurogenesis requires TopBP1 to prevent catastrophic replicative DNA damage in early progenitors. Nature neuroscience 15, 819-826, doi:10.1038/nn.3097 (2012).

27 Lang, P. Y. et al. ATR maintains chromosomal integrity during postnatal cerebellar neurogenesis and is required for medulloblastoma formation. Development 143, 40384052, doi:10.1242/dev.139022 (2016).

28 Zhong, Y. et al. The level of origin firing inversely affects the rate of replication fork progression. The Journal of cell biology 201, 373-383, doi:10.1083/jcb.201208060 (2013).

29 Conti, C. et al. Replication fork velocities at adjacent replication origins are coordinately modified during DNA replication in human cells. Molecular biology of the cell 18, 30593067, doi:10.1091/mbc.E06-08-0689 (2007).

30 Courbet, S. et al. Replication fork movement sets chromatin loop size and origin choice in mammalian cells. Nature 455, 557-560, doi:10.1038/nature07233 (2008).

31 O'Donnell, M., Langston, L. \& Stillman, B. Principles and concepts of DNA replication in bacteria, archaea, and eukarya. Cold Spring Harbor perspectives in biology 5 , doi:10.1101/cshperspect.a010108 (2013).

32 Bell, S. P. \& Kaguni, J. M. Helicase loading at chromosomal origins of replication. Cold Spring Harbor perspectives in biology 5, doi:10.1101/cshperspect.a010124 (2013).

33 Montagnoli, A. et al. Identification of $\mathrm{Mcm} 2$ phosphorylation sites by S-phase-regulating kinases. The Journal of biological chemistry 281, 10281-10290, doi:10.1074/jbc.M512921200 (2006). 
34 Ilves, I., Petojevic, T., Pesavento, J. J. \& Botchan, M. R. Activation of the MCM2-7 helicase by association with Cdc45 and GINS proteins. Molecular cell 37, 247-258, doi:10.1016/j.molcel.2009.12.030 (2010).

35 Pruitt, S. C., Bailey, K. J. \& Freeland, A. Reduced Mcm2 expression results in severe stem/progenitor cell deficiency and cancer. Stem cells 25, 3121-3132, doi:10.1634/stemcells.2007-0483 (2007).

36 Macheret, M. \& Halazonetis, T. D. Intragenic origins due to short G1 phases underlie oncogene-induced DNA replication stress. Nature 555, 112-116, doi:10.1038/nature25507 (2018).

37 Tubbs, A. et al. Dual Roles of Poly(dA:dT) Tracts in Replication Initiation and Fork Collapse. Cell 174, 1127-1142 e1119, doi:10.1016/j.cell.2018.07.011 (2018).

38 Hiratani, I. et al. Global reorganization of replication domains during embryonic stem cell differentiation. PLoS Biol 6, e245, doi:10.1371/journal.pbio.0060245 (2008).

39 Macheret, M. \& Halazonetis, T. D. Monitoring early S-phase origin firing and replication fork movement by sequencing nascent DNA from synchronized cells. Nat Protoc 14, 5167, doi:10.1038/s41596-018-0081-y (2019).

40 Sukup-Jackson, M. R. et al. Rosa26-GFP direct repeat (RaDR-GFP) mice reveal tissueand age-dependence of homologous recombination in mammals in vivo. PLoS Genet 10, e1004299, doi:10.1371/journal.pgen.1004299 (2014).

41 Cavalli, F. M. G. et al. Intertumoral Heterogeneity within Medulloblastoma Subgroups. Cancer cell 31, 737-754 e736, doi:10.1016/j.ccell.2017.05.005 (2017).

42 Tamayo-Orrego, L., Swikert, S. M. \& Charron, F. Evasion of cell senescence in SHH medulloblastoma. Cell cycle, 1-6, doi:10.1080/15384101.2016.1189044 (2016).

43 Archer, T. C. et al. Proteomics, Post-translational Modifications, and Integrative Analyses Reveal Molecular Heterogeneity within Medulloblastoma Subgroups. Cancer cell 34, 396-410 e398, doi:10.1016/j.ccell.2018.08.004 (2018).

44 Iwai, K. et al. Molecular mechanism and potential target indication of TAK-931, a novel CDC7-selective inhibitor. Sci Adv 5, eaav3660, doi:10.1126/sciadv.aav3660 (2019).

45 Siddiqui, K., On, K. F. \& Diffley, J. F. Regulating DNA replication in eukarya. Cold Spring Harbor perspectives in biology 5, doi:10.1101/cshperspect.a012930 (2013).

46 Bartkova, J. et al. Oncogene-induced senescence is part of the tumorigenesis barrier imposed by DNA damage checkpoints. Nature 444, 633-637, doi:10.1038/nature05268 (2006).

47 Viale, A. et al. Cell-cycle restriction limits DNA damage and maintains self-renewal of leukaemia stem cells. Nature 457, 51-56, doi:10.1038/nature07618 (2009).

48 Walter, D. et al. Exit from dormancy provokes DNA-damage-induced attrition in haematopoietic stem cells. Nature 520, 549-552, doi:10.1038/nature14131 (2015).

49 Bester, A. C. et al. Nucleotide deficiency promotes genomic instability in early stages of cancer development. Cell 145, 435-446, doi:10.1016/j.cell.2011.03.044 (2011).

50 Bartek, J., Lukas, C. \& Lukas, J. Checking on DNA damage in S phase. Nature reviews. Molecular cell biology 5, 792-804, doi:10.1038/nrm1493 (2004).

51 Maya-Mendoza, A. et al. High speed of fork progression induces DNA replication stress and genomic instability. Nature 559, 279-284, doi:10.1038/s41586-018-0261-5 (2018).

52 Anglana, M., Apiou, F., Bensimon, A. \& Debatisse, M. Dynamics of DNA replication in mammalian somatic cells: nucleotide pool modulates origin choice and interorigin spacing. Cell 114, 385-394 (2003). 


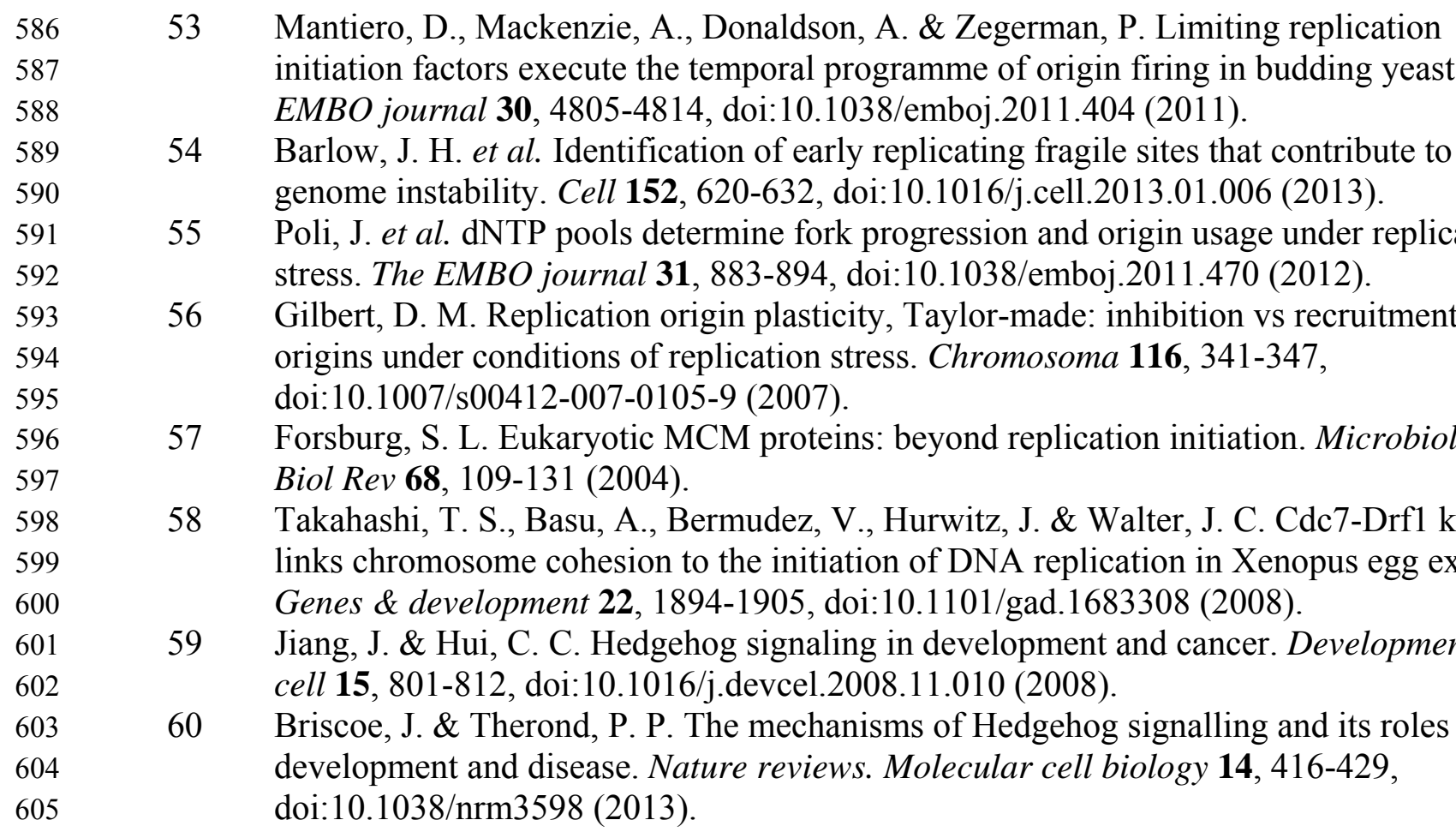


Fig. 1. CNVs and copy neutral LOH are the source of PTCH1 wild-type allele inactivation. a, Example of copy-neutral-LOH (CN-LOH) in human SHH-MB responsible for inactivation of the PTCH1 wild-type allele. Top plot shows absolute copy number and lower plot displays allele-specific copy number; left plots display all chromosomes while the right ones display chromosome 9 (a,b). Schematic shows the homologous recombination event that leads to $\mathrm{CN}-$ LOH. After DNA replication and a recombination event between the two homologous chromosomes, the $\mathrm{LOH}$ event is produced in mitosis depending on how sister chromatids are segregated. $\mathbf{b}$, Schematic and example of CNV-LOH in SHH-MB responsible for inactivation of the PTCH1 wild-type allele. Schematic shows the CNV (deletion) LOH event. c, Minimal segment of LOH on mouse chromosome 13 from 9 Ptch $^{+/-}$advanced MBs based on SNP analysis; 7/9 samples display chromosome $13 \mathrm{LOH}$ (absence of SNPs). d, CNV represented as segments of gain or loss (SGOL score) in advanced Ptch $1^{+/}$MBs show high level of deletion at the Ptch1 locus without broad chromosome 13 deletions. e, qPCR on genomic DNA from 3 advanced $P t c h 1^{+/}$MBs demonstrated biallelic loss of the wild-type Ptch1 allele; the primers recognize the gene region (Exon1-2) targeted in the engineered mutant allele and are therefore specific for the Ptch1 wild-type allele ${ }^{9}$, detecting Ptch1 LOH; shown are mean Ptch1 allele levels; each point represents one animal. f, Metaphases labelled with Chromosome 11 (red) and

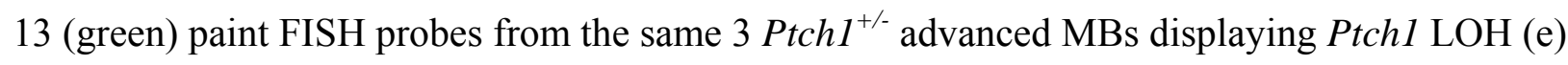
show absence of whole chromosome 13 losses or big deletions, consistent with CN-LOH. Scale bar, $5 \mu \mathrm{m}$; each (M1-M3) tumor was stained independently once and 30 metaphases/tumor analyzed.

Fig. 2. Shh causes DNA replication stress. a, $\gamma$-H2AX levels in BrdU-positive (BrdU+) and BrdU-negative (BrdU-) GCPs; one-way anova, median and Tukey distribution, $\mathrm{n}=248$ cells for 
Ctl samples and $n=203$ cells for Shh samples; data from 3 independent experiments. b, BrdU

641

642

643

644

645

646

647

648

649

650

651

652

653

654

655

656

657

658

659

660

661

662 and $\gamma$-H2AX immunostaining; histograms show mean fluorescence along a cross-section of a GCP (white line); $n=3$ experiments. Throughout the paper, $\gamma-\mathrm{H} 2 \mathrm{AX}$ levels were obtained by measuring the average nuclear fluorescence intensity. c, 53bp1 immunofluorescence and proportion of 53bp1-positive GCPs in BrdU- and BrdU+ cells; $n=422$ cells (378 BrdU- and 44 BrdU+) from 3 independent experiments; two-sided Fisher's exact test; arrowheads indicate $53 \mathrm{bp} 1$ foci. d, Number of 53bp1 foci in BrdU- and BrdU+ GCPs; mediantinterquartile range, two sided Mann-Whitney test , $n=122$ BrdU+ cells and $n=39$ BrdU- cells from 3 independent experiments; cells without foci were not analyzed in d since they are better represented in c. e, BrdU and $\gamma$-H2AX immunofluorescence of GCPs treated with vehicle $(\mathrm{Ctl}), 100 \mathrm{ng} / \mathrm{ml}$ Igf1, or 10nM Shh; $n=3$ experiments. f, Quantification of BrdU incorporation (mean $\pm 95 \% \mathrm{CI}), \mathrm{n}=25$ images for Ctl, $n=29$ images for Igf1, and n= 20 images for Shh samples, representative of 3 independent experiments. g, Quantification of $\gamma$-H2AX levels in Ctl-, Igf1- and Shh-treated GCPs; median and Tukey distribution; one-way anova (f,g); $n=146 \mathrm{Ctl}$ cells, $\mathrm{n}=249$ Igf-treated cells, and n= 359 Shh-treated cells from 3 experiments (e-g). h, Quantification and representative images of chromatin-bound Rpa32 in S-phase (BrdU+) GCPs, two-sided t-test, mean \pm 95\% CI; $\mathrm{n}=93 \mathrm{Ctl}$ S-phase cells, and n= 110 Shh S-phase cells from 3 experiments; scale bars, $5 \mu \mathrm{m}$ (b,c,e,h).

Fig. 3. Shh alters DNA replication dynamics. a, Schematic of GCP isolation, ex-vivo culture for $24 \mathrm{hr}$. , DNA combing experiments and variables measured. b, Quantification of replication fork asymmetry in Ctl-, Igf1- and Shh-treated GCPs; median with Tukey distribution, KruskalWallis test, $\mathrm{n}=330$ bi-directional forks for $\mathrm{Ctl}, \mathrm{n}=227$ bidirectional forks for Igfl, and $\mathrm{n}=185$ 
bidirectional forks for Shh samples. Data from 3 independent experiments. c, DNA replication fork speed (kbp/min) in Ctl-, Igf- and Shh-treated GCPs; median (M) and number (n) of fibers indicated; median and inter-quartile range, Kruskal-Wallis test, $\mathrm{n}=1064$ forks for Ctl samples, 582 forks for Igf1 samples, and 609 forks for Shh samples; obtained from 3 experiments. d, DNA fork density (Forks/Mbp DNA) in Ctl-, Igf- and Shh-treated GCPs; 207Mb, 203Mb and $230 \mathrm{Mb}$ of combed DNA measured for Ctl, Igfl and Shh conditions respectively, obtained from 2 independent experiments. e, Representative images of replication forks analyzed, $\mathrm{n}=3$ experiments. f,g, Experimental outline (f) and results (g) of S-phase time (hr.) measurements in Ctl- and Shh-treated GCPs; mean \pm sem. ( $\mathrm{n}=4$ experiments), two-sided t-test. h, Representation of the DNA replication changes induced by Shh in comparison to oncogenes. i, Comparison between Shh-induced replication stress (Shh-RS) and oncogene-induced replication stress (OIRS).

Fig. 4. Shh promotes pre-replication complex assembly. a, GSEA plot for the pre-replicative complex in Shh- vs. Ctl-treated GCPs; $\mathrm{n}=3$ samples/group. b,c, Nuclear (Nucl.) and chromatinbound (Chrom.) Mcm2 levels in Ctl- and Shh-treated GCPs assessed by immunofluorescence, $\mathrm{n}=190 \mathrm{Ctl}$ cells and $\mathrm{n}=207$ Shh cells (nuclear images); $\mathrm{n}=176 \mathrm{Ctl}$ cells and $\mathrm{n}=207$ Shh cells (Chromatin faction) from 3 independent experiments; mean $\pm 95 \% \mathrm{CI}$, two-sided t-test. Scale bar, $5 \mu \mathrm{m}$. d, Chromatin-bound Mcm7 levels in Ctl- and Shh-treated GCPs; mean $\pm 95 \%$ CI two-sided t-test, $\mathrm{n}=346$ cells for $\mathrm{Ctl}$ samples and $\mathrm{n}=363$ cells for Shh samples, from 3 experiments. e, Western blot (WB) from Ctl-, Igf- and Shh-treated GCPs subjected to cell fractionation and blotted for Mcm2, B-tubulin and histone H2B; TCE, total cell extract; S2, cytosol; P2, membrane; S3, nuclear soluble; $\mathrm{P} 3$, chromatin. Mean Mcm2 levels relative to $\mathrm{H} 2 \mathrm{~B}$ in $\mathrm{P} 3$ are 
indicated, two sided t-test; $n=3$ experiments. f, Chromatin-bound Mcm2 in G0/G1 and S-phase $(\mathrm{BrdU}+)$ in Ctl- and Shh-treated GCPs; mean $\pm 95 \% \mathrm{CI}$, two-tailed t-test; $\mathrm{n}=166$ cells for $\mathrm{Ctl}$ and $n=186$ cells for Shh samples $(\mathrm{G} 0 / 1)$ and $n=46$ cells for $\mathrm{Ctl}$ and $n=109$ cells for Shh samples (Sphase) from 3 independent experiments. g, Chromatin-bound Mcm2 in early and late S-phase in Ctl- and Shh-treated GCPs; representative data of $\mathrm{n}=3$ experiments, mean $\pm 95 \% \mathrm{CI}$, two-sided $\mathrm{t}$ test; $\mathrm{n}>40$ S-phase cells for Ctl samples and $\mathrm{n}>90$ S-phase cells for Shh samples, from 2 independent experiments. h, Representative images of chromatin-bound Mcm2 of GCPs in different cell cycle stages; $n \geq 4$ experiments; scale bars, $2 \mu \mathrm{m}$. i, Model showing the effect of Shh

696 697 Fig. 5. Shh induces helicase activation and origin firing. a,b, pMcm2 s40/41 and Mcm2 WB promoting helicase loading.

(a) and quantification (b) from GCPs treated with Ctl, Cdc7i (PHA767491, 1 $\mu \mathrm{M})$, Shh or Shh+Cdc7; mean \pm sem., anova test; $n=4$ blots for Ctl and Shh samples, $n=2$ blots for Cdc $7 i, n=$ 3 blots for Shh+Cdc7i condition. c, Experimental outline of fork density measurements (d); bottom images show the total DNA stain (ssDNA) corresponding to images shown in e. d, Fork density (forks/Mbp) in Ctl-, Cdc7i-, Shh- and Shh+Cdc7i-treated GCPs (759Mb, 1028Mb, $650 \mathrm{Mb}$ and $924 \mathrm{Mb}$ of combed DNA respectively, analyzed from two experiments). e, Representative images of fork density measurements shown in d; scale bar, $50 \mu \mathrm{m}(\mathrm{c}, \mathrm{e})$. f,g, Representative images of inter-origin distance (IOD) (f) and quantitation (g) in Ctl-, Cdc7i-, Shhand Shh+Cdc7i-treated GCPs; Kruskal-Wallis test, median with Tukey distribution; $\mathrm{n}=86,30$, 153 and 75 fibers for the respective Ctl-, Cdc7i-, Shh- and Shh+Cdc7i-treated samples; data from 2 independent experiments; scale bar, $10 \mu \mathrm{m}(\mathrm{f}) . \mathbf{h}$, IODs in $\mathrm{Mcm} 2^{+/+}$and $\mathrm{Mcm} 2^{+/-}$GCPs treated 
without or with Shh; one-way anova, median with Tukey distribution. The "n" of DNA fibers scored was: $\mathrm{Mcm}^{+/+} \mathrm{Ctl}, \mathrm{n}=40 ; \mathrm{Mcm}^{+/+}+\mathrm{Shh}, \mathrm{n}=56 ; \mathrm{Mcm}^{+/-} \mathrm{Ctl}, \mathrm{n}=57 ; \mathrm{Mcm}^{+/-}+\mathrm{Shh}, \mathrm{n}=$ 78; data from 2 independent experiments. Median IODs indicated below graphs (g,h). Cdc7i was added during the last $4 \mathrm{hr}$ of the experiments (a-g). i, Model summarizing Shh effects on replication initiation.

\section{Figure 6. Shh-dependent replication initiation domains revealed by EdU-seq. a, Examples} of Edu-seq tracks in Ctl- and Shh-treated GCPs. Input is the first row of each replicate. Left graphs depict common replication initiation zones and right graphs illustrate Shh-dependent initiation zones; $\mathrm{n}=2$ experiments. $\mathbf{b}$, Percentage of EdU-seq peaks localized to early replicating regions in Ctl- and Shh-treated GCPs. c, Number of CNVs in Ptch1 ${ }^{+/-}$MBs that overlap (or not) with EdU-seq peak-containing regions in Ctl- and Shh-treated GCPs; two-sided Fisher's exact test; $\mathrm{n}=942$ peaks for Ctl samples, and $\mathrm{n}=4321$ peaks for Shh samples, from two independent experiments were analyzed (b-c). d, EdU-seq tracks of Ctl-, Shh-, and Shh+Cdc7i-treated GCPs; $\mathrm{n}=2$ experiments.

\section{Fig. 7. Origin firing is required for Shh-dependent replication stress and recombination. a,} Images of $\gamma$-H2AX immunostaining in Ctl-, Cdc7i-, Shh- and Shh+Cdc7i-treated GCPs; right side panels show merge $\gamma-\mathrm{H} 2 \mathrm{AX}, \mathrm{BrdU}$ and Dapi images; $\mathrm{n}=3$ experiments. b, Quantification of $\gamma-\mathrm{H} 2 \mathrm{AX}$ images presented in $\mathrm{d}, \mathrm{n}=612$ cells for $\mathrm{Ctl}, \mathrm{n}=52$ cells for $\mathrm{Cdc} 7 \mathrm{i}, \mathrm{n}=680$ cells for Shh, $\mathrm{n}=748$ cells for $\mathrm{Shh}+\mathrm{Cdc} 7 \mathrm{i}$, from 3 independent experiments; median, boxplots with Tukey 
distribution; one-way anova with Tukey post-test. c, Chromatin-bound Rpa32 (ssDNA) in Sphase (BrdU+ cells) in Ctl-, Igf1-, Cdc7i-, Shh- and Shh+Cdc7i-treated GCPs; the number of Sphase cells was: $n=65$ in Ctl, $n=45$ in Igfl, $n=43$ in Cdc7i, $n=66$ in Shh, $n=48$ in Shh+Cdc7i S-phase cells from 2 representative experiments; mean $\pm 95 \% \mathrm{CI}$, one way anova, Tukey post-test

(b,c). d, Representative images of c. e, Experimental outline and quantification of sister chromatid exchanges (SCEs)/metaphase in Ctl-, Igf1-, Shh- and Shh+Cdc7i-treated GCPs; the numbers of metaphases analyzed were: $n=90$ in Ctl, $n=98$ in Igfl, $n=87$ in Shh, and $n=54$ in Shh+Cdc7i; data from of 3 independent experiments; median and Tukey distribution; KruskalWallis test. f, Images of metaphases labeled with the SCE assay in Ctl-, Igf1-, Shh- and Shh + Cdc7i-treated GCPs; $n=3$ experiments. Scale bars (a,d,f), $5 \mu \mathrm{m}$. In a-d, Cdc7i was added during the last $4 \mathrm{hr}$ of the experiment.

\section{Fig. 8. In vivo Cdc7 inhibition reduces origin firing and hyper-recombination, preventing} medulloblastoma initiation. a, Experimental design. Ctl- and Cdc7i (TAK-931)-treated Ptch $^{+/-}$mice were analyzed at postnatal day 7 (P7) and P16. b, Mouse body weight at P12 in Ctl- and Cdc7i-treated pups; $\mathrm{n}=14$ mice in Ctl group and $\mathrm{n}=22$ mice in Cdc7i group; mean \pm sem, two-sided t-test. c-d, Cerebellum area $\left(\mathrm{mm}^{2}\right)$ at midline (c) and representative images (d) in Ctland Cdc7i-treated P16 mice; mean \pm sem, two-sided t-test, $n=6$ mice/group. e, Latency to fall on rotarod test; mean \pm sem, two tailed t-test, $n=9$ mice/group. f, $p$-Mcm2 levels in the outer EGL of Ctl- and Cdc7i-treated mice; mean \pm sem, two-sided t-test, $\mathrm{n}=5$ mice/group. $\mathbf{g}$, BrdU fluorescence intensity in Ctl- and Cdc7i-treated mice, mean \pm sem; two-tailed t-test, $\mathrm{n}=5$ animals/group. $\mathbf{h}$, Representative images of BrdU and p-Mcm2 staining, $n=5$ animals; scale bar, $3 \mu \mathrm{m}$. i, Outline of 
in vivo DNA combing and fork density quantification of Ctl- and Cdc7i-treated mice for 6 consecutive days and analyzed at P7, n=3 animals/group; two-sided t-test, mean \pm sem. $\mathbf{j}$, Experimental outline of in vivo recombination assay and number of SCEs/metaphase in vivo in Ctl- and Cdc7i-treated mice at P7; median with range, Mann-Whitney test; $n=57$ metaphases in Ctl and n=69 metaphases in Cdc7i-treated mice, analyzed from 3 experiments. Representative images shown on the right; scale bar, $2 \mu \mathrm{m}$. $\mathbf{k}$, Preneoplastic lesion incidence at P16 in Ctl- and Cdc7i-treated mice; two-sided Fisher's exact test. l, Number of preneoplastic lesions per cerebellum in Ctl- and Cdc7i-treated mice; mean \pm sem, two-sided t-test; $\mathrm{n}=11 \mathrm{Ctl}$ mice, and $\mathrm{n}=$ 14 Cdc7-treated mice $(k, 1)$. m, Examples of H\&E P16 cerebellum sections of Ctl- and Cdc7itreated mice shown in 1,k. Scale bar, $500 \mu \mathrm{m}$. n, Model of Shh-dependent replication stress and medulloblastoma initiation I: Physiological levels of Shh increase both active origin number and fork speed, resulting in accelerated S-phase. This however leads to increased replication stress, likely due to reduced time to solve challenges to DNA replication, increasing recombination events. II: In wild-type GCPs Shh-replication stress and increased recombination rates alone are not sufficient to cause biallelic inactivation of Ptch1. III: However, in In Ptch1 ${ }^{+/}$GCPs, Shhdriven recombination events causing Ptch1 LOH are sufficient to cause tumor suppressor inactivation $\left(P t c h 1^{-1}\right)$, leading to constitutive, ligand-independent, hedgehog pathway activation that perpetuates mitogenic stimulation resulting in clonal expansion of the Ptch1 $1^{-/}$GCPs; this in turn causes preneoplasia. Continued replication stress at preneoplastic stages would further promote genome instability and progression to advanced tumors. 


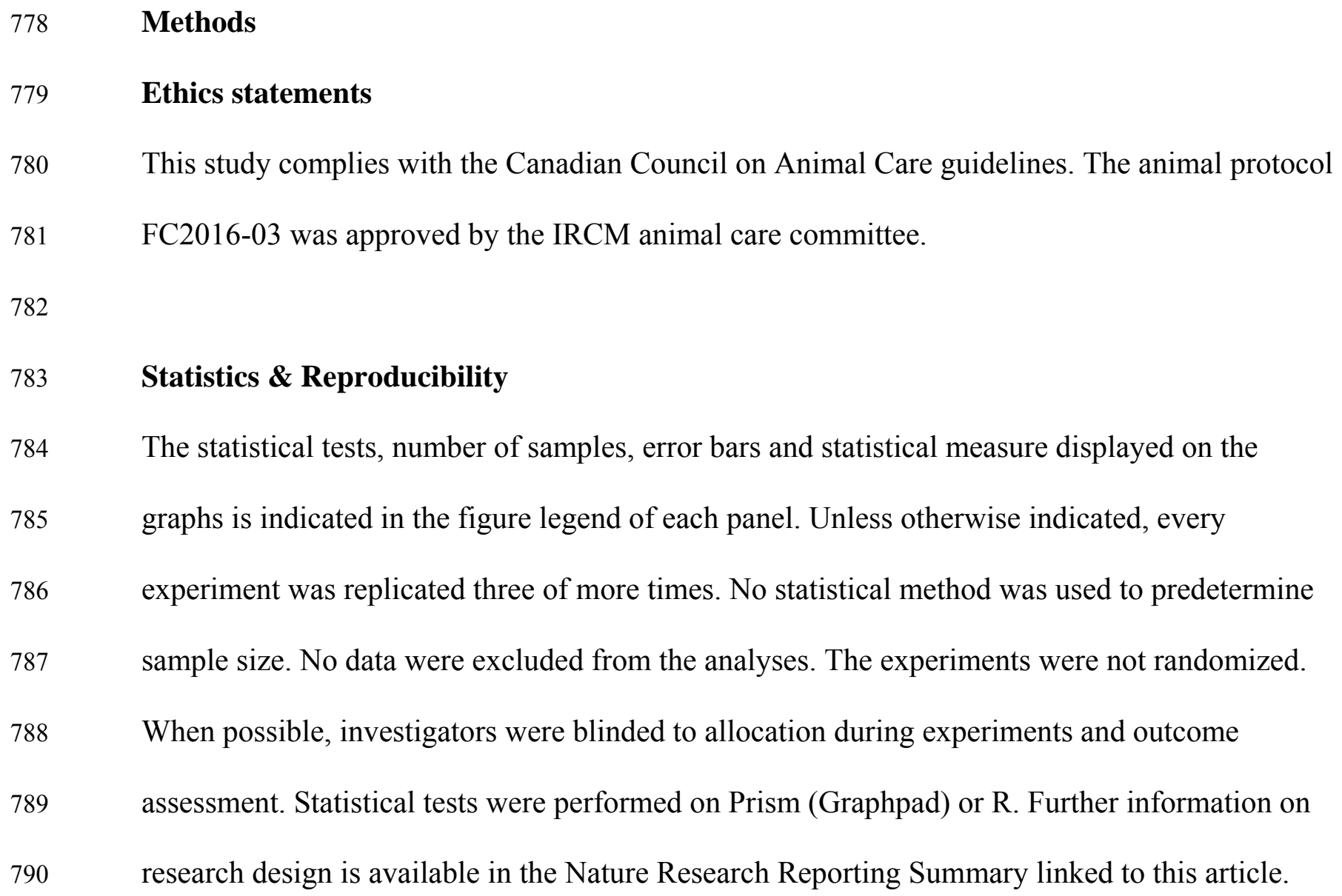

Postnatal day 7 (P7) GCPs were isolated as previously described ${ }^{61}$.

For most experiments, GCPs were grown in serum-free Neurobasal media supplemented with B27, Glutamax and Sodium Pyruvate for 24hrs. Murine Igf1 (PreproTech, Cat. No. 250-19) was used at 100ng/ml, and Shh (R\&D Systems, Cat. No. 1845-SH) at 10nM.

\section{Immunofluorescence (IF)}


For dissociated cells, GCPs were fixed in 4\% PFA by adding 8\% PFA in 1X PBS to the same volume of media for $15 \mathrm{~min}$. Immunofluorescence was performed as previously described ${ }^{24,61}$. To visualize chromatin-bound proteins, GCPs were incubated for $5 \mathrm{~min}$ at $4^{\circ} \mathrm{C}$, before fixation, in extraction buffer containing 25mM Hepes, $\mathrm{pH} 7.5,50 \mathrm{mM} \mathrm{NaCl}, 1 \mathrm{mM}$ EDTA, 3mM magnesium chloride, $300 \mathrm{mM}$ glucose and $0.5 \%$ Triton $\mathrm{X}-100{ }^{62}$. After blocking in $10 \%$ goat or donkey serum containing $0.1 \%$ Triton, cells were incubated in primary antibodies overnight at $4^{\circ} \mathrm{C}$. The following antibodies were used: Rat anti-BrdU (Abcam, Cat. No. ab6326), 1:2000. Mouse anti-BrdU clone B44 (BD, Cat. No. 347580), 1:200. Anti-RPA32 (Abcam, Cat. No. 16855), 1:100. Anti- $\gamma$-H2AX (CST, Cat. No. 9718S), 1:2000. Anti- $\gamma$-H2AX clone JBW301 (Millipore, Cat. No. 05-636), 1:1000. Anti- $\gamma$-H2AX-AF647 (Biolegend Cat. No. 613408), 1:200. Anti p-MCM2 S40/S41 (Bethyl, Cat. No. A300-788-M), 1:200. Anti-MCM2 (Abcam Cat. No. ab4461) 1:2000. Anti-53BP1 (Novus, Cat. No. NB100-304), 1:4000. Anti-Rad51 (Abcam, Cat. No. ab133534), 1:1000. 53BP1 and Rad51 immunostainings required antigen retrieval for $15 \mathrm{~min}$ at $98^{\circ} \mathrm{C}$ in sodium citrate buffer $(10 \mathrm{mM}$ sodium citrate $\mathrm{pH} 6.0,0.05 \%$ Tween 20$)$ before the blocking step. All secondary antibodies were used at a 1:1000 dilution. Image acquisition was performed on a LSM700 (Zeiss), a LSM800 Airy Scan (Zeiss), or a SP8 (Leica) confocal microscope.

\section{DNA combing}

For each experimental condition (e.g. control, Igf1, Shh, nucleosides), 1.2x10 7 P7 GCPs were cultured in $60 \mathrm{~mm}$ dishes in replicate for 24 hours. Cells were pulsed with $25 \mathrm{mM}$ CldU (Sigma, Cat. No. C6891) for 20min, washed with pre-warmed PBS and then pulsed with $125 \mathrm{mM}$ IdU (Sigma, Cat. No. I7125) for 20min. GCPs were harvested by pipetting. $5 \times 10^{6}$ GCPs were used to 
cast 3 agarose (Bioshop, AGA101) plugs per replicate and processed for DNA combing according to a previously described protocol ${ }^{63}$ omitting the SCE buffer plug digestion steps. IdU was detected using Mouse anti-BrdU clone B44 (BD, Cat. No. 347580) and CldU was detected using Rat anti-BrdU (Abcam, Cat. No. ab6326). Images were acquired using a widefield microscope (Leica DM4000 or Zeiss Axiophot) using a 63X or 40X lens. The elongation rate ( $\mu \mathrm{m}$ to kbp conversion) was obtained after bacteriophage lambda DNA combing, as previously described ${ }^{63}$. Measurements were performed using ImageJ. Fork density was calculated by counting the number of labeled DNA replication forks per mega-base pair of DNA (Mbp), which was measured using anti ssDNA antibody (MAB 3034) staining; all ssDNA fibers were measured independent of whether they contained labelled forks. Fork density was normalized to S-phase (Fig. 5d) by dividing fork density values by the S-phase fraction. For DNA fork speed, the length of the IdU tracks next to CldU tracks was measured and divided by the IdU incubation time (20min). DNA fork asymmetry is expressed as \% asymmetry=((long IdU - short IdU)1)*100. IODs correspond to "eye-to-eye" distances (expressed in kbp), as exemplified in Fig. $5 \mathrm{f}-\mathrm{h}$.

\section{Cell fractionation}

Cell fractionation was performed according to a modified protocol ${ }^{64} .1 .2 \times 10^{7} \mathrm{P} 7 \mathrm{GCPs}$ were used per condition. Protein lysates were loaded on pre-casted TGX gels for SDS-PAGE and western blotting. Anti-histone H2B (Abcam, Cat. No. ab1790), 1:25,000. Anti-B-tubulin, 1:10,000. Anti-Mcm2 (Abcam Cat. No. ab4461) 1:10,000.

\section{GCP metaphase preparations}




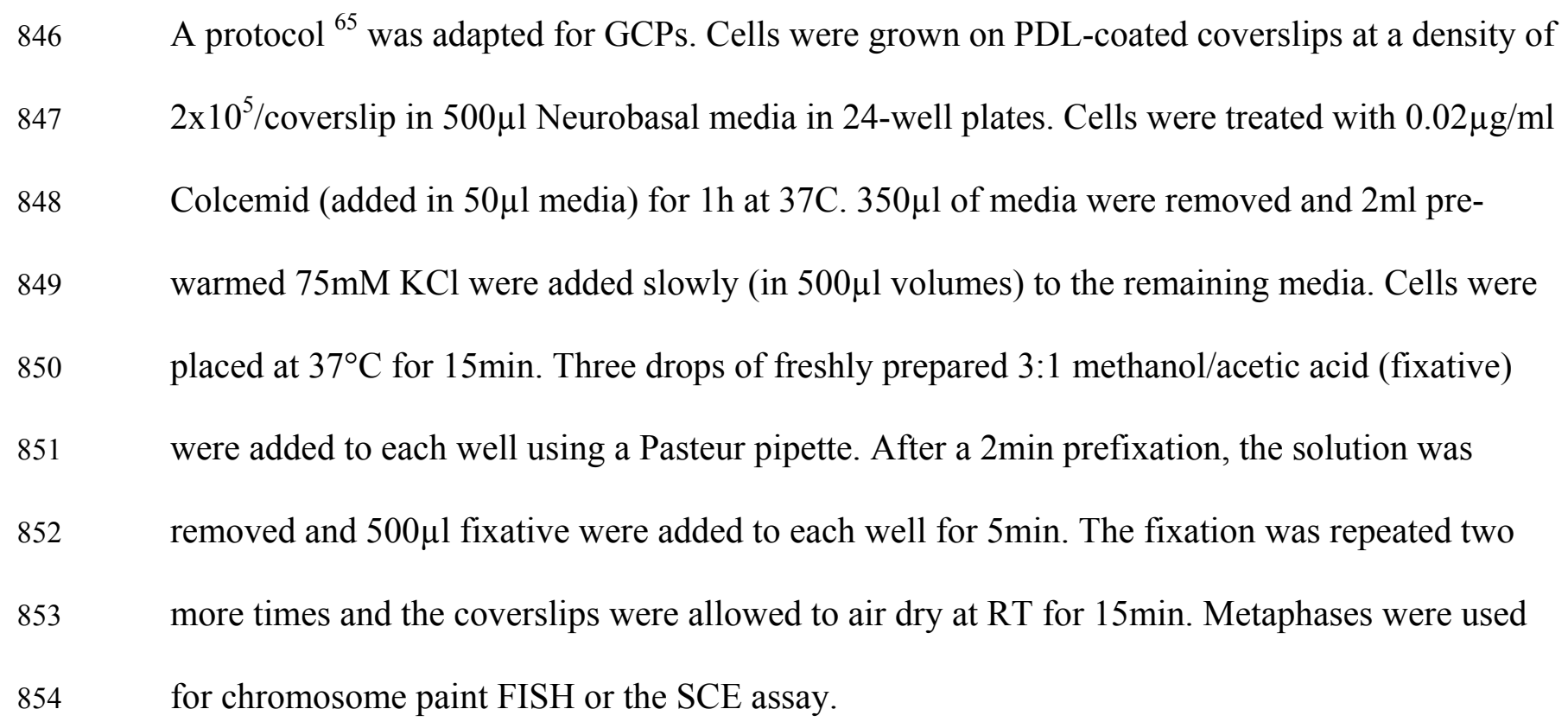


S-phase time measurements were performed using sequential pulses of thymidine analogs ${ }^{67}$ in order to extrapolate the percentage of cells in S-phase by measuring the Leave or Quit fraction, the fraction of cells that exit S-phase in a determined time window according to the formula $\mathrm{Ts}=$ Ti*Scells/Lcells, were Ti represents the time (hrs) between pulses, Scells are cells in S-phase and Lcells are the Leave fraction. GCPs were pulsed with $25 \mu \mathrm{M} \mathrm{CldU}$ for $2 \mathrm{hr}$ and during the last $30 \mathrm{~min}$ of that period a $30 \mathrm{~min}$ pulse of $125 \mu \mathrm{M}$ IdU was added and cells were fixed in $4 \%$ PFA. Rat anti-BrdU (Abcam, Cat. No. ab6326) was used to recognize CldU-positive cells (Lcells), while mouse anti-BrdU clone B44 (BD, Cat. No. 347580) was used to label IdU-positive cells (Scells).

\section{Nucleoside supplementation}

Intermediate dilutions were prepared at a $50 \mathrm{mM}$ concentration. Thymidine and cytidine were dissolved in $\mathrm{H}_{2} \mathrm{O}$, while adenosine and guanosine were dissolved in $20 \mathrm{mM} \mathrm{NaOH}$. The solutions were mixed in equal proportions to obtain a final stock solution containing $2.5 \mathrm{mM}$ of each nucleoside; this solution was further diluted in the culture media to $5 \mu \mathrm{M}$.

\section{In vivo Cdc7 inhibition}

Newborn Ptch1 $1^{+/}$mice were treated daily from postnatal day 1 (P1) to postnatal day 16 (P16) with $2 \mathrm{mg} / \mathrm{kg}$ TAK-931(Cdc7i) by intraperitoneal injections; the injection volume was $10 \mu 1$. Control mice were injected daily with a vehicle solution containing $1.25 \%$ DMSO in water. The side of the injection was alternated every day. To avoid litter effects, each litter always contained animals being treated with vehicle and animals assigned to Cdc7i. At P16, mice were 
anesthetized and perfused with saline and 4\% PFA. Cerebella were dissected, sectioned and stained with Hematoxylin \& Eosin.

\section{In vivo DNA combing}

Postnatal 7 (P7) pups that had been treated for seven days with vehicle or TAK-931 were given an intraperitoneal (IP) dose of $100 \mathrm{mg} / \mathrm{kg}$ CldU for $20 \mathrm{~min}$ followed by a $100 \mathrm{mg} / \mathrm{kg}$ pulse of IdU for $20 \mathrm{~min}$. All mice were sacrificed at the same time and GCPs were quickly isolated, directly embedded in agarose plugs and processed for DNA combing as explained above.

\section{In vivo recombination assay}

Ctl- or Cdc7i-treated postnatal day 7 (P7) pups were given intraperitoneal injections with $50 \mathrm{mg} / \mathrm{Kg}$ BrdU every hour for $7 \mathrm{hr}$. Three hours after the last BrdU injection a dose of $2 \mathrm{mg} / \mathrm{kg}$ TAK-931 or vehicle was administered to extend the treatment regime for an additional day (pups were left with their mother), and GCPs were isolated at P8, plated on PDL-coated coverslips for one hour, and the metaphases prepared as explained above.

\section{RNA sequencing (RNAseq) and analysis}

$3 \times 10^{6} \mathrm{GCPs} /$ well were grown in 6-well plates and treated for 24h with 1:1000 DMSO (Control), Igf1 and Shh in triplicate. Total RNA was extracted using the RNeasy Plus Mini Kit (Qiagen, Cat. No. 74134). 400-450ng total RNA were used for RNAseq. Enrichment of transcriptomic RNA was achieved by ribosomal RNA depletion using the Ribo-Zero Gold H/M/R rRNA removal kit from Epicentre (Illumina). Stranded RNA-Seq (chemical fragmentation, cDNAdsDNA, library) was performed using the KAPA Stranded RNA-Seq Library Preparation Kit 
913 (Illumina Platforms). Library quality control and quantitation were performed using the Agilent 914 High Sensitivity DNA kit on a Bioanalyzer and the NEBNext Library Quant Kit for Illumina. 915 Paired-End 50 reads were sequenced on a HiSeq 2500 with v4 chemistry (Illumina). 916 Quality control of read sequences was performed using the FastQC algorithm. Adaptor 917 sequences and low quality score bases (Phred score $<30)$ were first trimmed using Trimmomatic 918 v. $0.22^{68}$, and reads smaller than $32-\mathrm{bp}$ long were discarded. The resulting reads were mapped to 919 the mouse genome assembly mm10 using STAR ${ }^{69}$. Only concordant mapped reads were 920 subsequently used. HTSeq $0.6 .1 \mathrm{p} 1{ }^{70}$ was used to obtain gene mapping-read counts based on 921 Ensembl (release 86) gene annotation downloaded from the UCSC genome browser ${ }^{71}$. 922 Transcript isoforms from single genes were merged using cufflink ${ }^{72}$, resulting in 48526 923 annotated genes. We further selected genes expressed above a nominal background level, defined 924 as more than 10 mapping reads in at least 2 replicates of either sample. This results in $x$ 925 expressed genes. The R Bioconductor package DESeq2 ${ }^{73}$ was used to normalize gene expression 926 levels and to investigate the differential expression of genes between groups. A q-value $<0.05$ 927 and a minimum expression fold-change of 2 were considered to select significantly differentially 928 expressed genes. Source data for the RNAseq analyses shown in Extended Data Fig. 2, Extended 929 Data Fig. 3, Extended Data Fig. 4, and Extended Data Fig. 7 can be found in Supplementary $930 \quad$ Table 1.

\section{EdU-seq}

933 GCPs were pulsed with $100 \mu \mathrm{M}$ EdU (Sigma, Cat No. 900584) for 15min, washed in warm PBS 934 for $3 \mathrm{~min}$, harvested and processed as described ${ }^{37}$. Azide-PEG3-Biotin Conjugate was obtained 935 from Sigma (Cat. No. Sigma 762024). We used $1.5 \times 10^{7}$ GCPs/condition and two different 
experiments were performed as replicates. Inputs correspond to biotinylated DNA before the streptavidin capture of EdU-substituted DNA. Libraries were prepared using the Kappa HyperPrep Kit according to manufacturer instructions, using 12 amplification cycles. Library quality control and sequencing were performed as described for RNAseq. The resulting reads were aligned to the mm10 mouse reference genome using Bowtie v2.3.1 $1^{74}$ with the following parameters: -q --fr --no-mixed --no-unal -local. The output sam files were filtered using samtools ${ }^{75}$ to remove alignments containing three or more mismatches. Two concurrent analyses were performed to identify enriched regions. (1) Each sample replicate was compared to sequenced input DNA using MACS v2.1.1.20160309 ${ }^{76}$ callpeak function with the parameters "-f BAMPE -g mm --broad -nomodel -B -q 0.05 ”. Replication initiation zones were defined by clustering enriched regions that are $120 \mathrm{~kb}$ of each other using the tool bedtools cluster ${ }^{77}$ with the parameters "bedtools cluster $-\mathrm{i}-\mathrm{d} 120000$ ". (2) Each sample replicate was compared to sequenced input DNA using MACS v2.1.1.20160309 callpeak function with the parameters "-f BAMPE -g mm --broad -nomodel -B -q 1". Regions with at least 3-fold enrichment against the input DNA were kept for the subsequent analyses. Replication initiation zones were defined by clustering enriched regions $50 \mathrm{~kb}$ of each other using bedtools cluster with the parameters "bedtools cluster $-\mathrm{i}-\mathrm{d} 50000$ “.

\section{DNA copy number variation (CNV) analysis of $P$ tch $1^{+/}$advanced medulloblastomas}

We used normalized $\log 2$ ratio (sample/reference) of the aCGH array dataset GSE19381 (samples GSM480969, GSM480970 and GSM480971) from GEO. The R package cghMCR was used to quantify segments of DNA copy across the samples. 
960 We downloaded human MB whole genome sequences ${ }^{8}$ from the ICGC dataset EGAD00001003127 (EGAS00001001953) and analyzed copy number and allele-specific copy number using FACETS ${ }^{78}$.

\section{Mass spectrometry}

$1.5 \times 10^{7}$ GCPs per condition (Ctl, Igfl, Shh) were grown for 24 in triplicate in $60 \mathrm{~mm}$ dishes.

Cells were washed in cold $150 \mathrm{mM}$ ammonium formate solution $\mathrm{pH}$ of 7.4 and then extracted in $600 \mathrm{uL} 31.6 \% \mathrm{MeOH} / 36.3 \%$ acetonitrile in $\mathrm{H}_{2} \mathrm{O}(\mathrm{v} / \mathrm{v})$. Cells were lysed and homogenized by bead-beating for 2 minutes at $30 \mathrm{~Hz}$ using a $5 \mathrm{~mm}$ metal bead in a TissueLyser II (Qiagen). Cell extracts were partitioned into aqueous and organic layers following dimethyl chloride treatment

For semi-quantitative targeted metabolite analysis of mono-, di-, and tri-phosphate nucleoside, samples were injected onto an Agilent 6430 Triple Quadrupole (Agilent Technologies, Santa Clara, CA, USA). Chromatography was achieved using a 1290 Infinity ultra-performance LC system (Agilent Technologies, Santa Clara, CA, USA) consisting of vacuum degasser, autosampler and a binary pump. Separation was performed on a Scherzo SM-C18 column $3 \mu \mathrm{m}$, $3.0 \times 150 \mathrm{~mm}$ (Imtakt Corp, JAPAN) maintained at $10^{\circ} \mathrm{C}$. The chromatographic gradient started at $100 \%$ mobile phase A ( $5 \mathrm{mM}$ ammonium acetate in water) with a 5 min gradient to $100 \% \mathrm{~B}$ (200 $\mathrm{mM}$ ammonium acetate in $20 \% \mathrm{ACN} / 80 \%$ water) at a flow rate of $0.4 \mathrm{ml} / \mathrm{min}$. This was followed 
by a $5 \mathrm{~min}$ hold time at $100 \%$ mobile phase B and a subsequent re-equilibration time (6 min) before next injection. $5 \mathrm{uL}$ of sample volume was injected for analysis. Sample temperature was maintained at $4^{\circ} \mathrm{C}$ before injection.

The mass spectrometer was equipped with an electrospray ionization (ESI) source and samples were analyzed in positive mode. Multiple reaction monitoring (MRM) transitions were optimized on standards for each metabolite quantitated. Gas temperature and flow were set at $350^{\circ} \mathrm{C}$ and 10 $1 /$ min respectively, nebulizer pressure was set at $40 \mathrm{psi}$ and capillary voltage was set at $3500 \mathrm{~V}$. Relative concentrations were determined by integrating the area under the curve for the quantifying MRM transition and compared to external calibration curves. Data were analyzed using MassHunter Quant (Agilent Technologies, Santa Clara, CA, USA).

\section{Data availability}

Deep-sequencing data supporting the findings of this study have been deposited in the Gene Expression Omnibus (GEO) under accession codes GSE147409 (EdU-seq) and GSE147410 (RNA-seq).

Human LOH data in SHH-MB were derived from dataset EGAD00001003127 obtained with authorization from the ICGC Consortium: https://icgc.org/. Mouse CNV data was downloaded from GSE19381. Source data for Fig. 1-8, Extended Data Fig. 1-10, and Uncropped Gels have been provided as Source Data Files. All other data supporting the findings of this study are available from the corresponding author on reasonable request.

\section{Methods-only references}


61 Izzi, L. et al. Boc and Gas1 each form distinct Shh receptor complexes with Ptch1 and are required for Shh-mediated cell proliferation. Developmental cell 20, 788-801, doi:10.1016/j.devcel.2011.04.017 (2011).

62 Lukas, C., Falck, J., Bartkova, J., Bartek, J. \& Lukas, J. Distinct spatiotemporal dynamics of mammalian checkpoint regulators induced by DNA damage. Nature cell biology 5, 255-260, doi:10.1038/ncb945 (2003).

63 Gallo, D., Wang, G., Yip, C. M. \& Brown, G. W. Analysis of Replicating Yeast Chromosomes by DNA Combing. Cold Spring Harb Protoc 2016, pdb prot085118, doi:10.1101/pdb.prot085118 (2016).

64 Mendez, J. \& Stillman, B. Chromatin association of human origin recognition complex, cdc6, and minichromosome maintenance proteins during the cell cycle: assembly of prereplication complexes in late mitosis. Molecular and cellular biology 20, 8602-8612 (2000).

65 Bayani, J. \& Squire, J. A. Preparation of cytogenetic specimens from tissue samples. Curr Protoc Cell Biol Chapter 22, Unit 22 22, doi:10.1002/0471143030.cb2202s23 (2004).

66 Hoch, N. C. et al. XRCC1 mutation is associated with PARP1 hyperactivation and cerebellar ataxia. Nature 541, 87-91, doi:10.1038/nature20790 (2017).

67 Martynoga, B., Morrison, H., Price, D. J. \& Mason, J. O. Foxg1 is required for specification of ventral telencephalon and region-specific regulation of dorsal telencephalic precursor proliferation and apoptosis. Dev Biol 283, 113-127, doi:10.1016/j.ydbio.2005.04.005 (2005).

68 Lohse, M. et al. RobiNA: a user-friendly, integrated software solution for RNA-Seqbased transcriptomics. Nucleic acids research 40, W622-627, doi:10.1093/nar/gks540 (2012).

69 Dobin, A. et al. STAR: ultrafast universal RNA-seq aligner. Bioinformatics 29, 15-21, doi:10.1093/bioinformatics/bts635 (2013).

70 Anders, S., Pyl, P. T. \& Huber, W. HTSeq--a Python framework to work with highthroughput sequencing data. Bioinformatics 31, 166-169, doi:10.1093/bioinformatics/btu638 (2015).

71 Dreszer, T. R. et al. The UCSC Genome Browser database: extensions and updates 2011. Nucleic acids research 40, D918-923, doi:10.1093/nar/gkr1055 (2012).

72 Trapnell, C. et al. Transcript assembly and quantification by RNA-Seq reveals unannotated transcripts and isoform switching during cell differentiation. Nat Biotechnol 28, 511-515, doi:10.1038/nbt.1621 (2010).

73 Love, M. I., Huber, W. \& Anders, S. Moderated estimation of fold change and dispersion for RNA-seq data with DESeq2. Genome biology 15, 550, doi:10.1186/s13059-0140550-8 (2014).

74 Langmead, B. \& Salzberg, S. L. Fast gapped-read alignment with Bowtie 2. Nat Methods 9, 357-359, doi:10.1038/nmeth.1923 (2012).

75 Li, H. et al. The Sequence Alignment/Map format and SAMtools. Bioinformatics 25, 2078-2079, doi:10.1093/bioinformatics/btp352 (2009).

76 Zhang, Y. et al. Model-based analysis of ChIP-Seq (MACS). Genome Biol 9, R137, doi:10.1186/gb-2008-9-9-r137 (2008). 
$1050 \quad 77$ Quinlan, A. R. \& Hall, I. M. BEDTools: a flexible suite of utilities for comparing

1051 genomic features. Bioinformatics 26, 841-842, doi:10.1093/bioinformatics/btq033

1052

1053

1054

1055 (2010).

78 Shen, R. \& Seshan, V. E. FACETS: allele-specific copy number and clonal heterogeneity analysis tool for high-throughput DNA sequencing. Nucleic acids research 44, e131, doi:10.1093/nar/gkw520 (2016).

1057

1058 
a ICGC_MB59: Copy number neutral-LOH
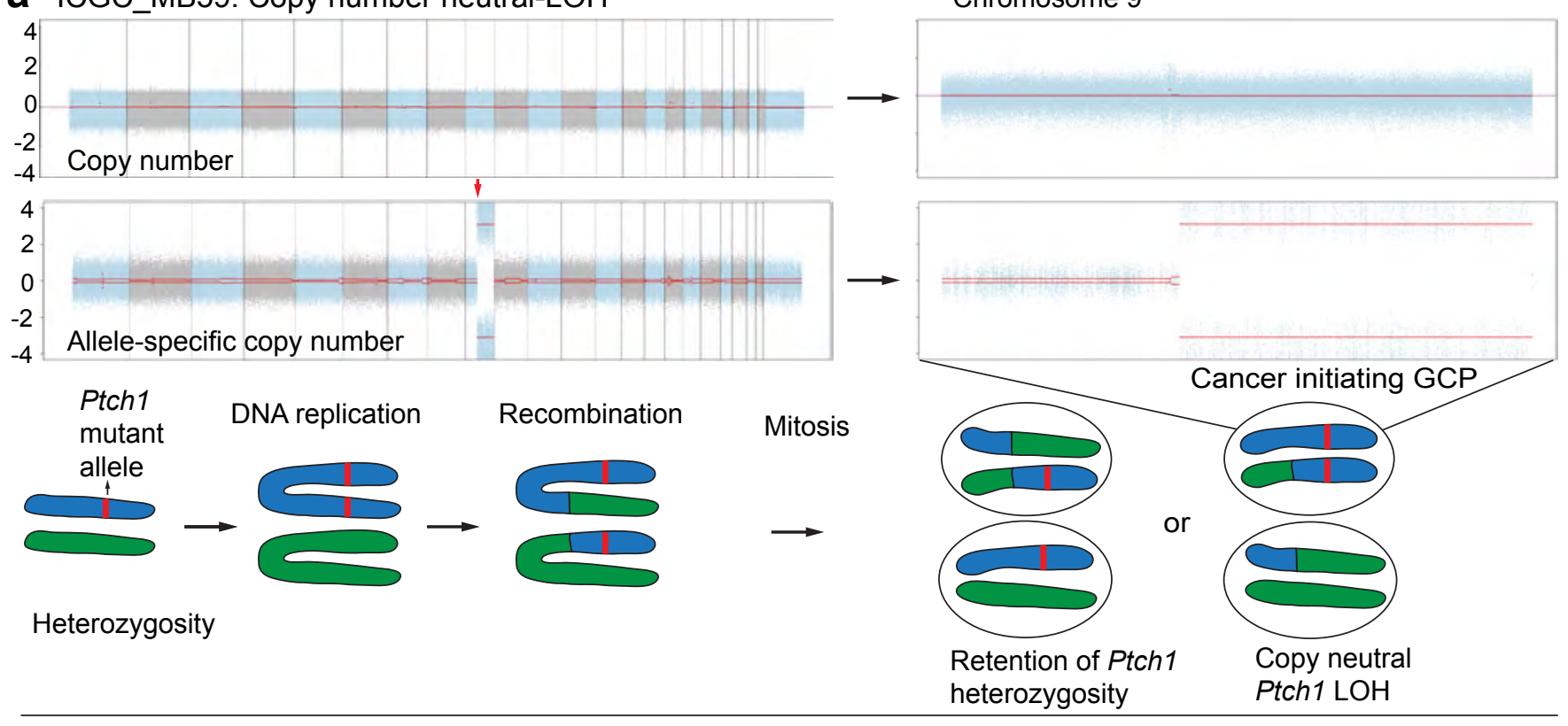

b Ptch1 mutant allele

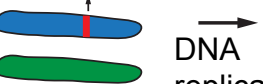

replication

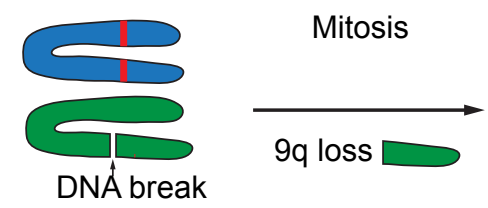

ICGC MB21: Copy number loss-LOH

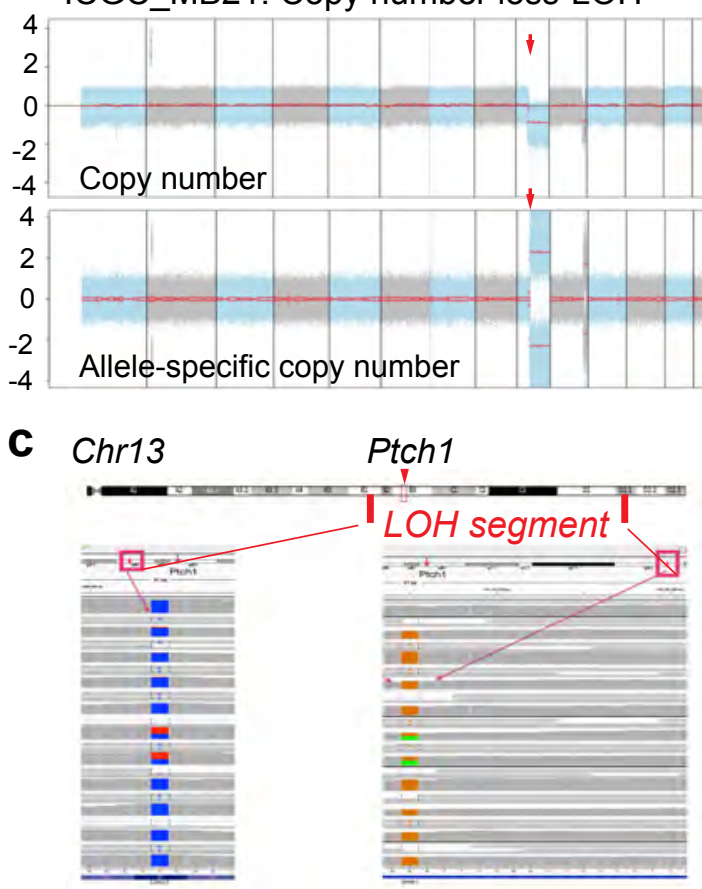

Chromosome 9
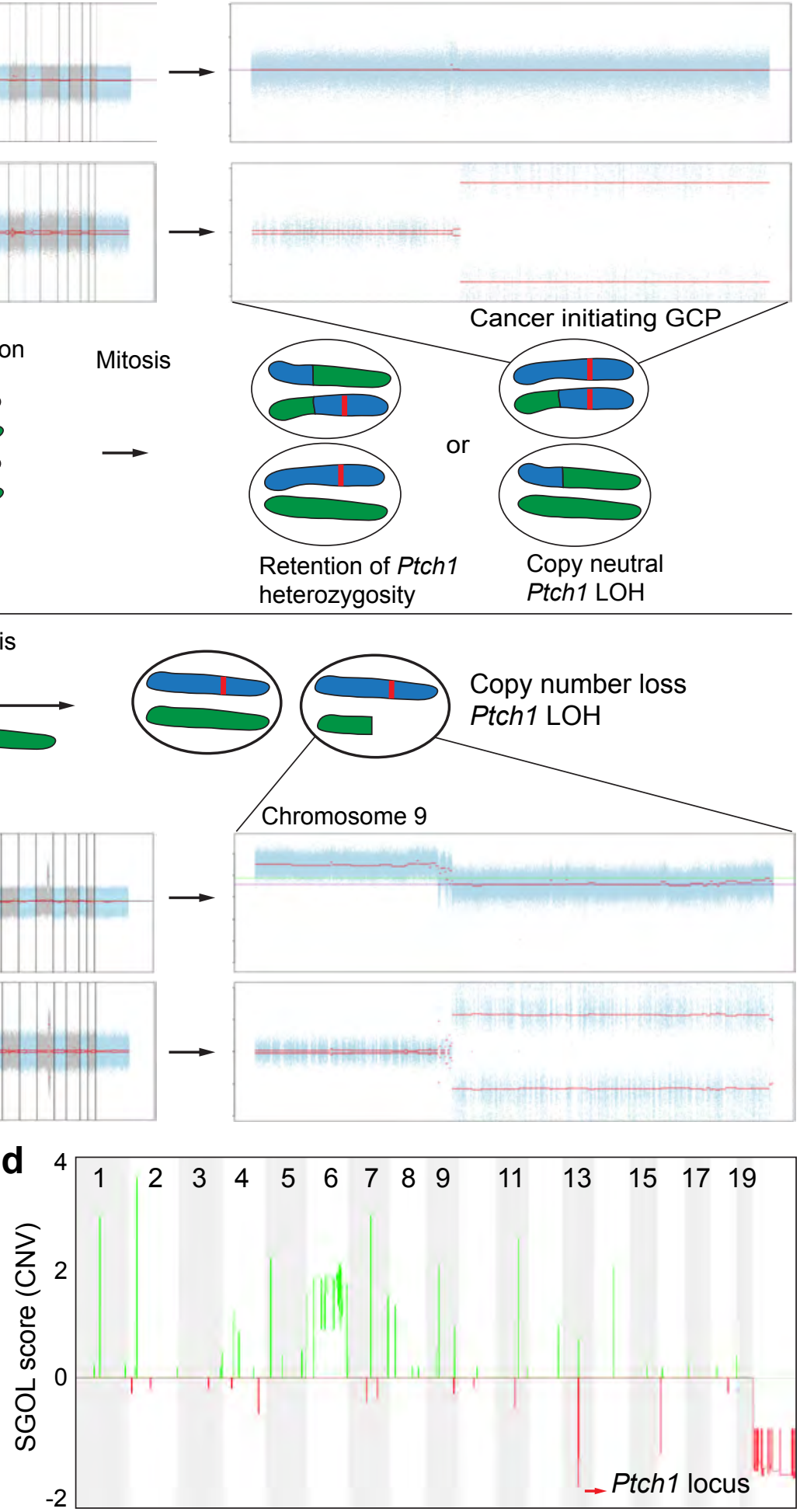

e
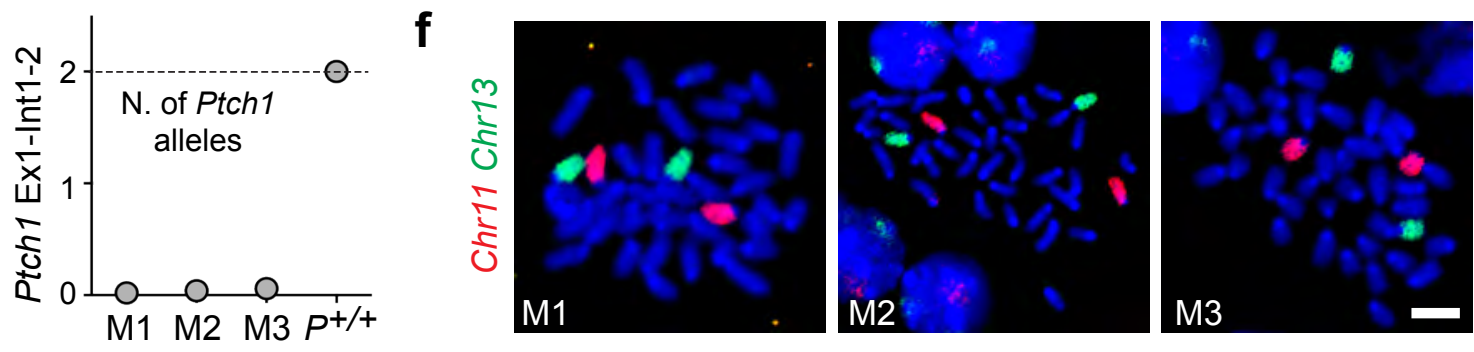


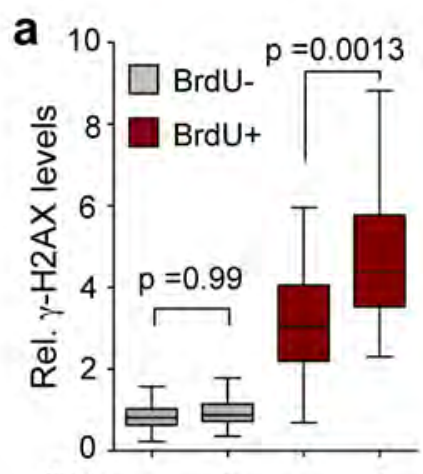

Shh: - + - +

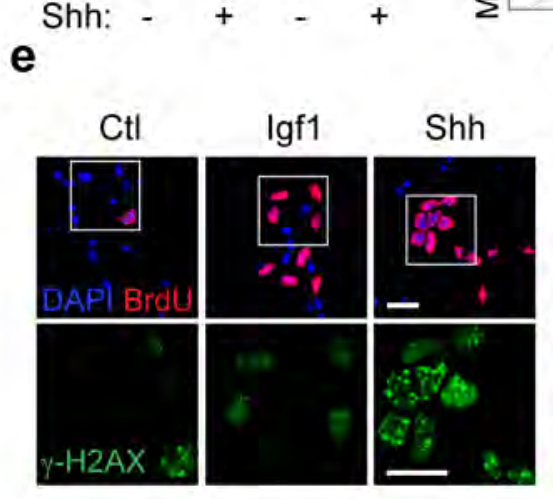

b
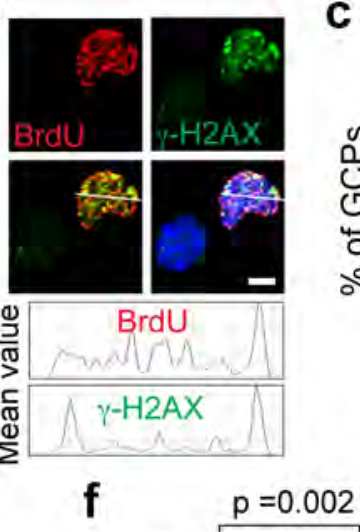

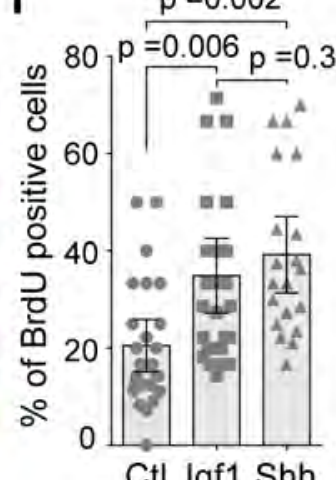

Ctl Igf1 Shh
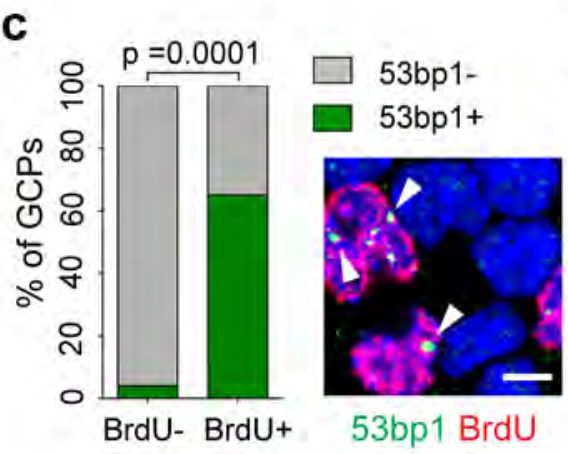
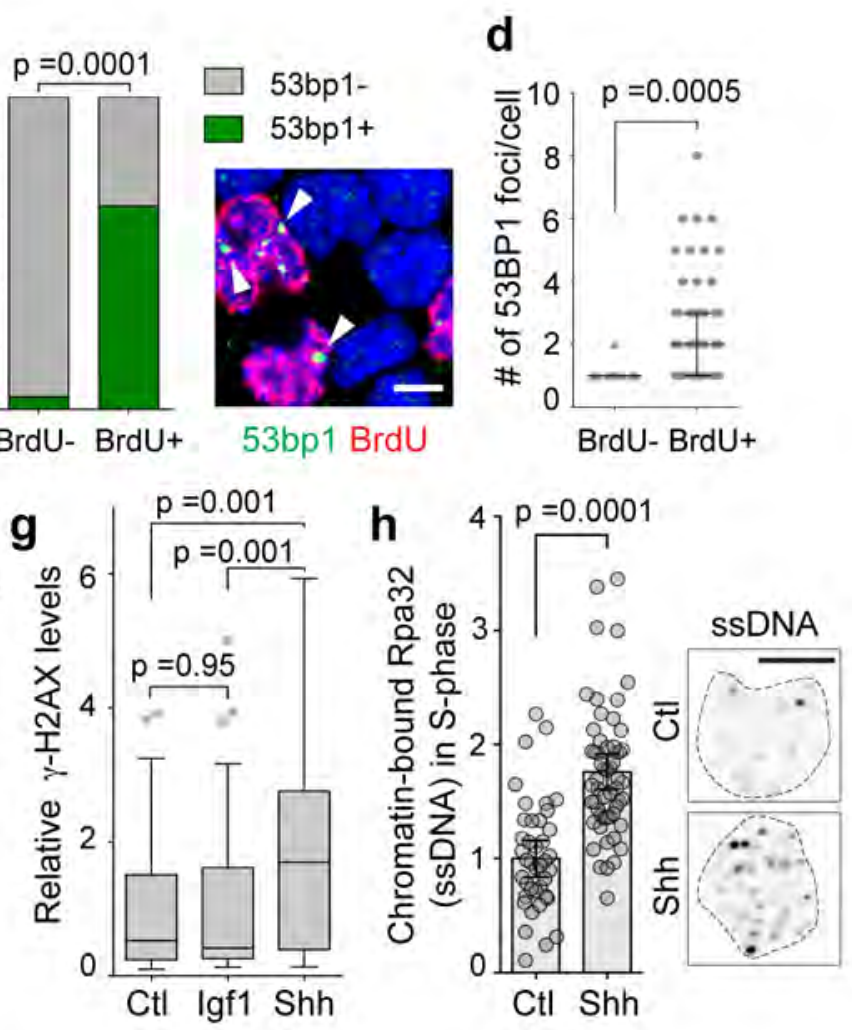
a

DNA combing of GCPs

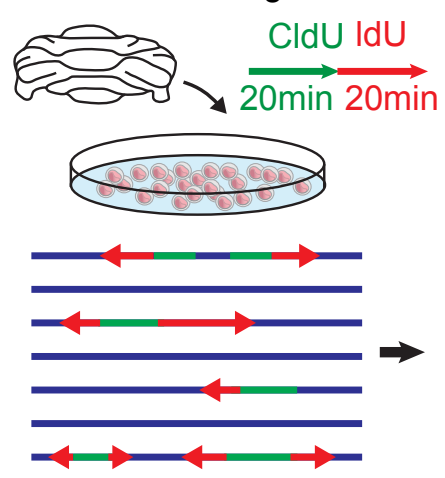

Fork speed

Asymmetry

Fork density

IODs b

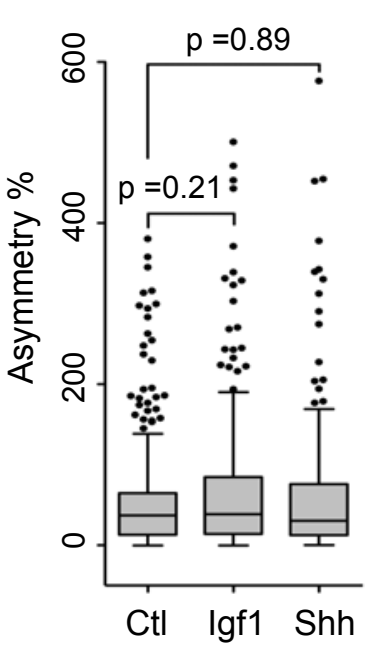

C

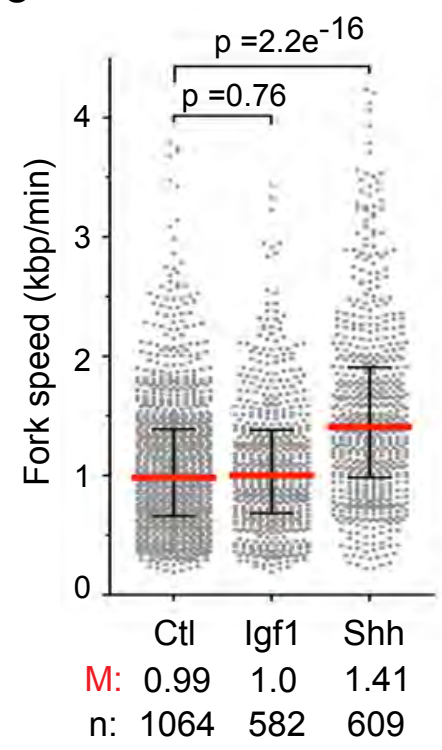

d

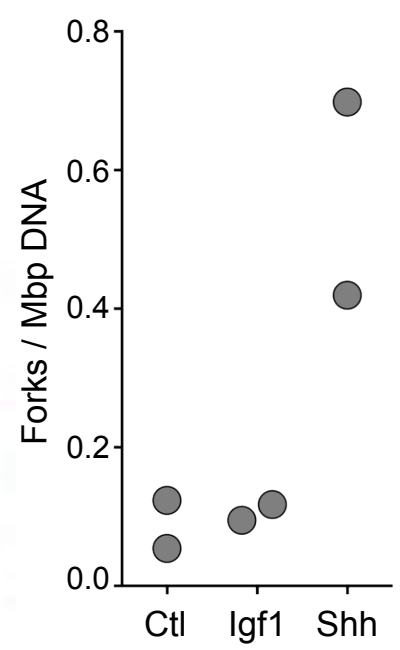

e

Ctl

$\operatorname{lgf1}$

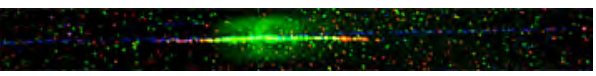

Shh

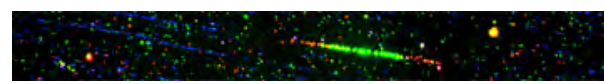

h

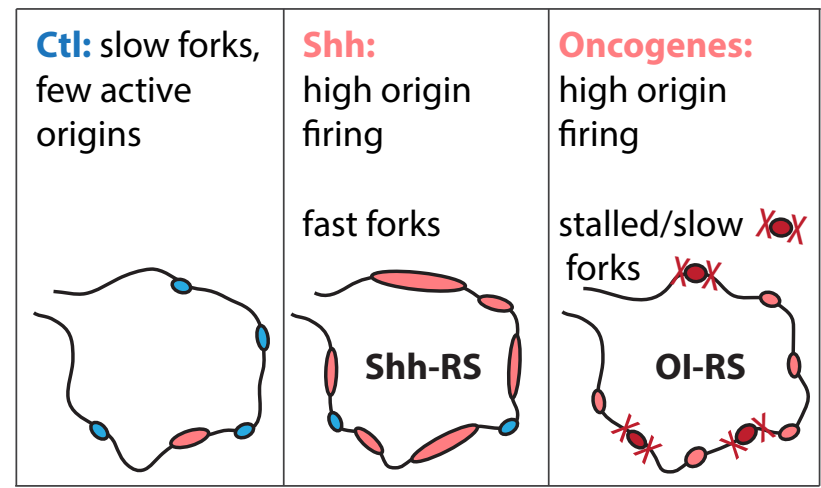

o Inactive origins f

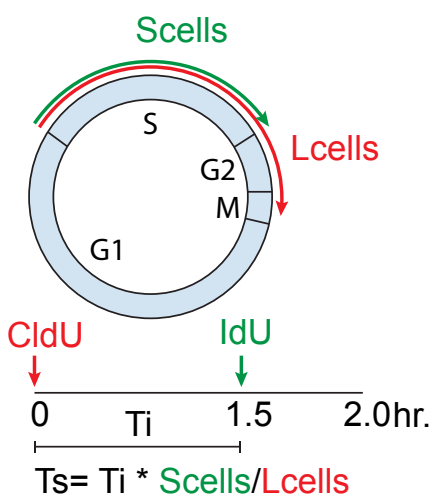

g

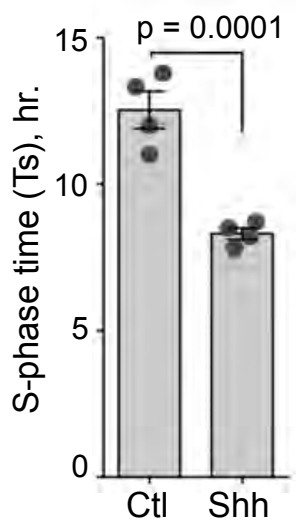

\section{i}

\section{Comparison of Shh-RS and OI-RS}

\begin{tabular}{|l|c|c|}
\hline & Shh-RS & Ol-RS \\
\hline Origin firing & High & Low or High \\
\hline Fork speed & Fast & Slow/stalling \\
\hline 1,2,3 \\
\hline dNTPs & High & Low $^{4}$ \\
\hline $\begin{array}{l}\text { Ultrafine anaphase } \\
\text { bridges (UFBs) }\end{array}$ & Not present & Present $^{5}$ \\
\hline 53bp1 foci & S-phase & G1 $^{6}$ \\
\hline
\end{tabular}

1. Macheret and Halazonetis, 2015

4. Bester et al., 2011

2. Bartkova et al., 2006; Di Mico et al., 2006

3. Dominguez-Sola et al., 2007

5. Chan et al., 2009

6. Lukas et al., 2011 

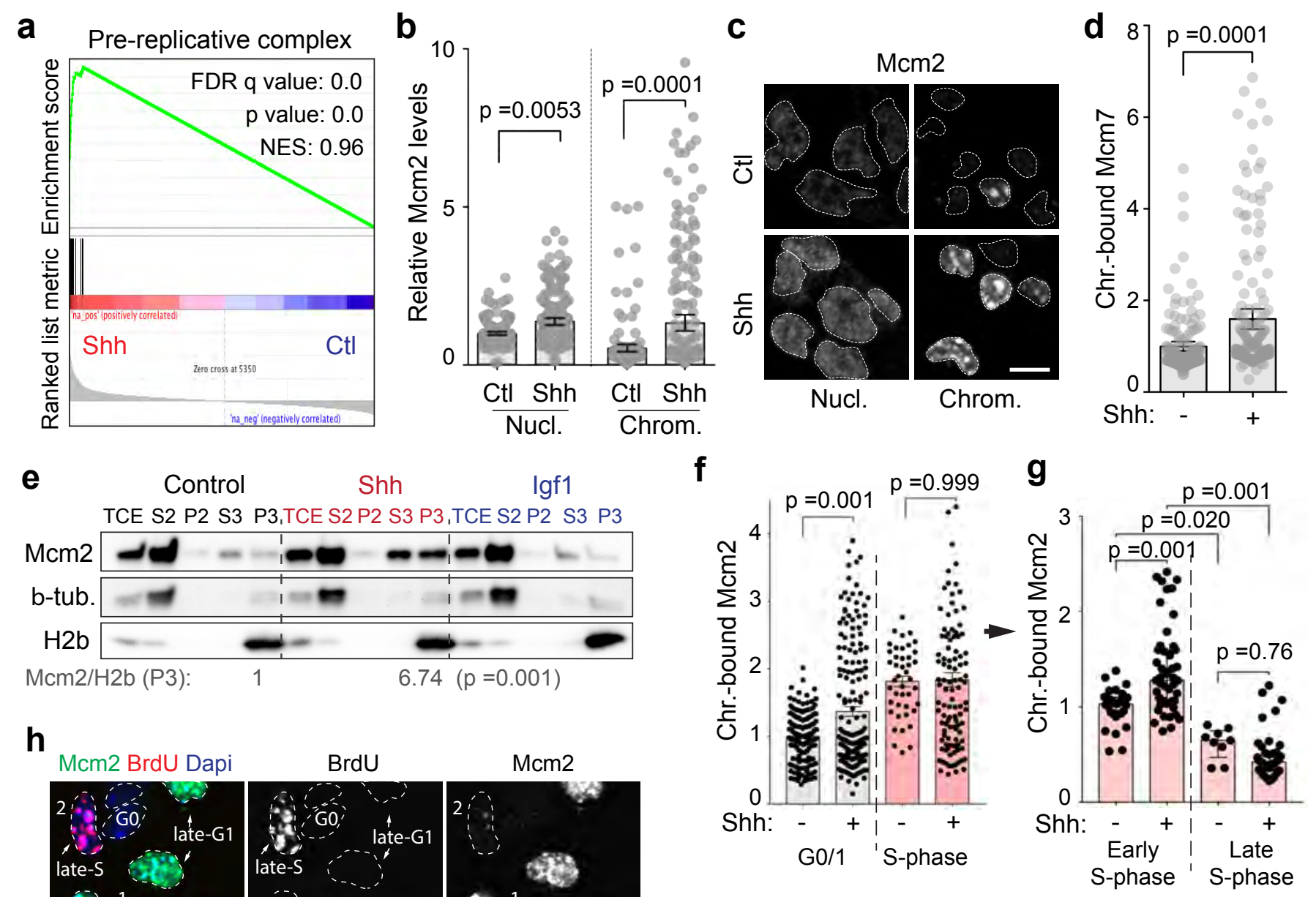

Mcm2/H2b (P3): $116.74(p=0.001)$

h
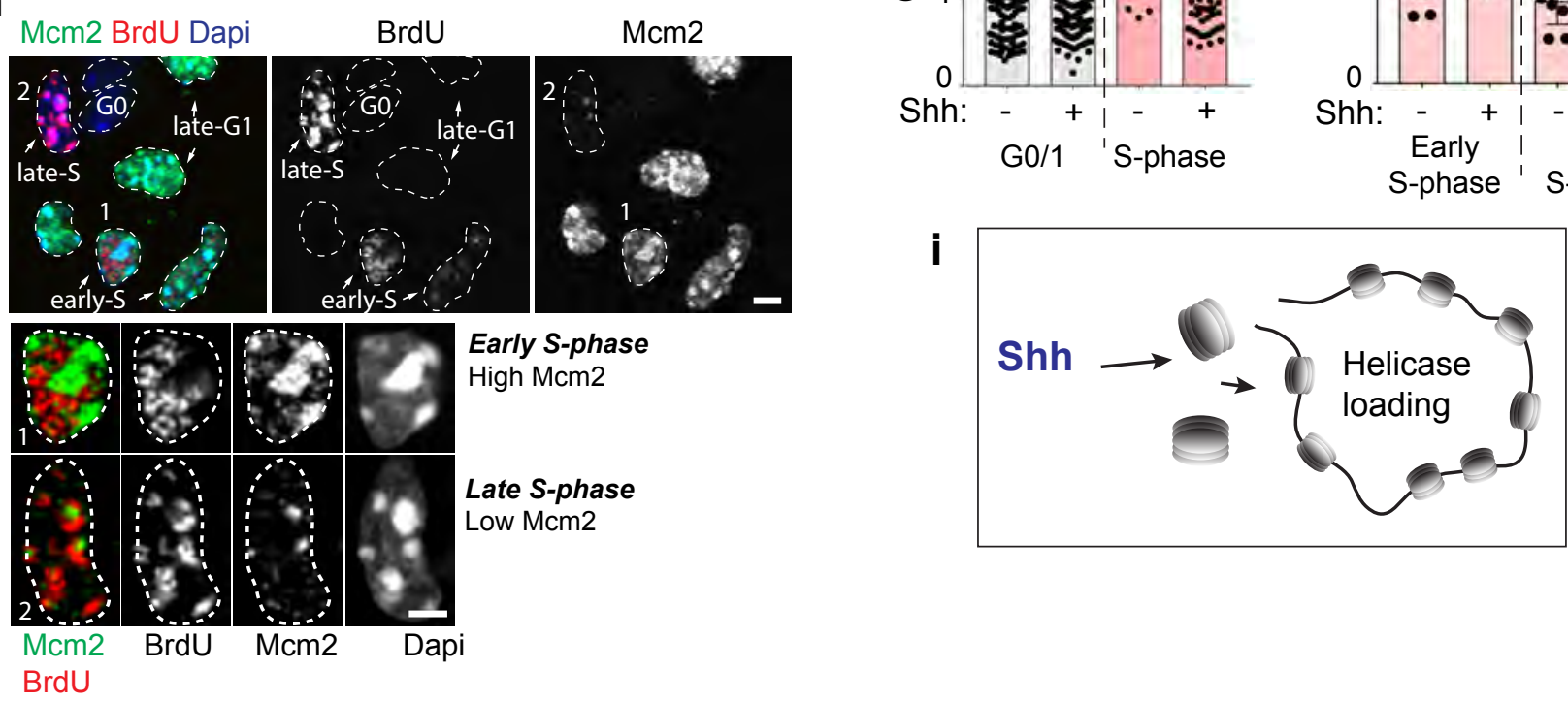
a

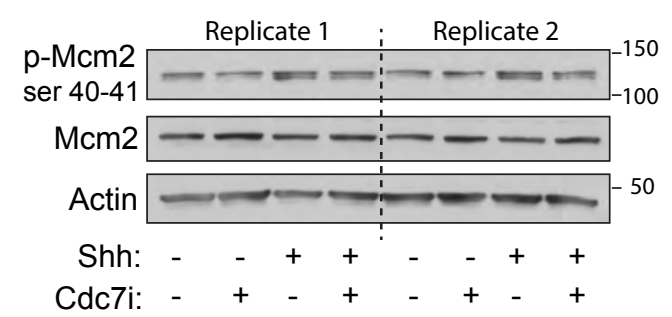

b

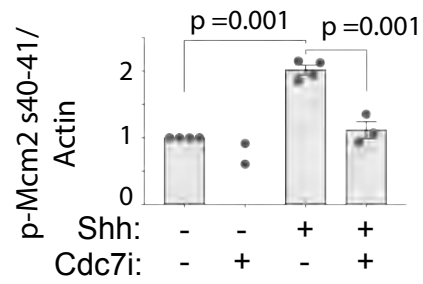

e

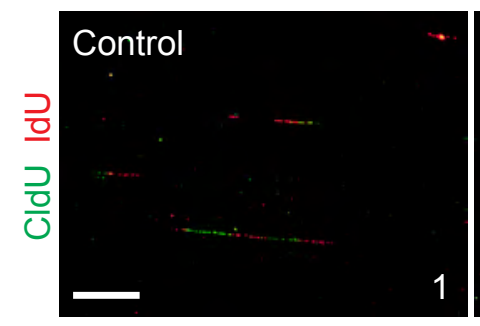

f

Inter-origin distance

Control

Cdc7i

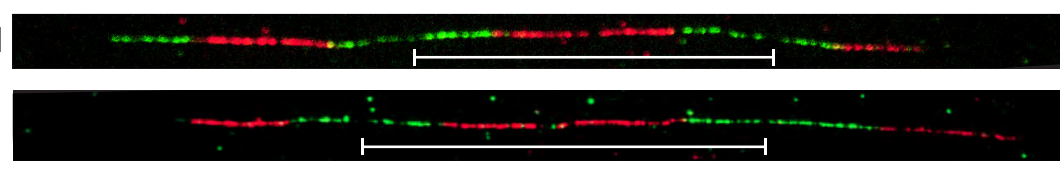

Shh
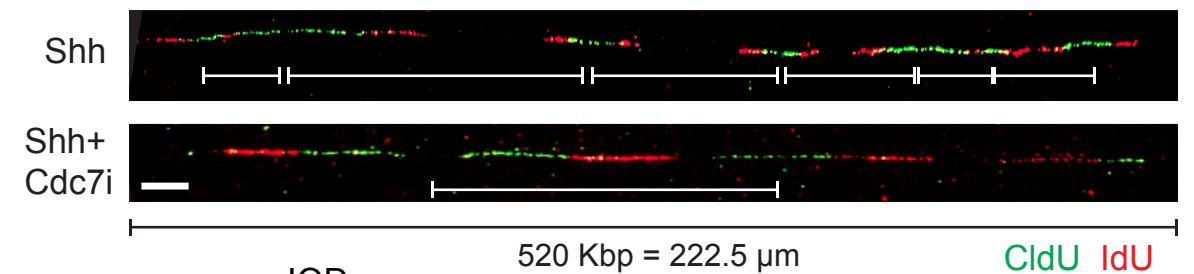

h
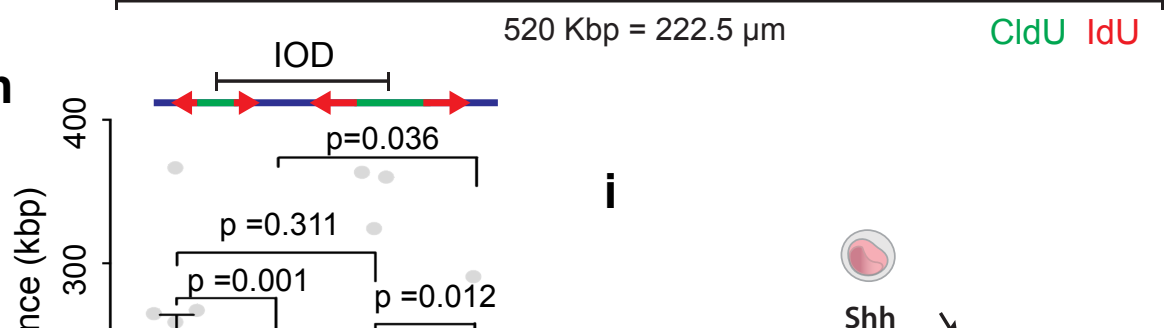$$
\text { 要 }
$$

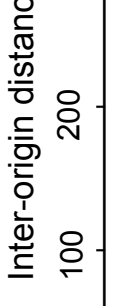

$$
\circ
$$

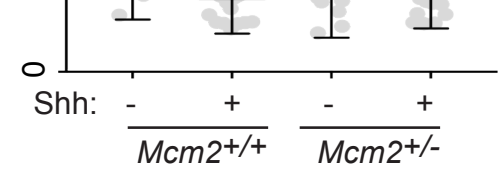

median: $134.1 \quad 66.95 \quad 120 \quad 96.25$
C

Fork density: Forks/DNA

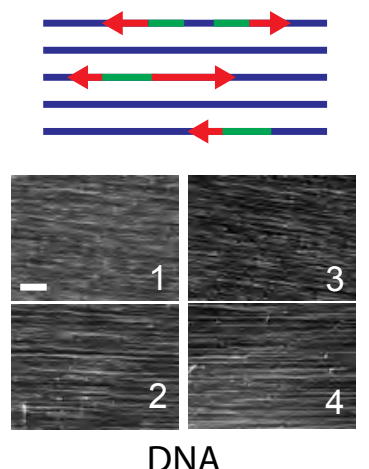

Fork density

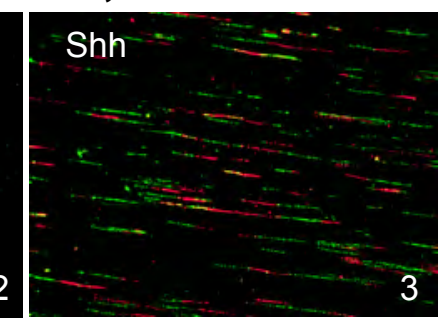

\section{Shh + Cdc7i}

g
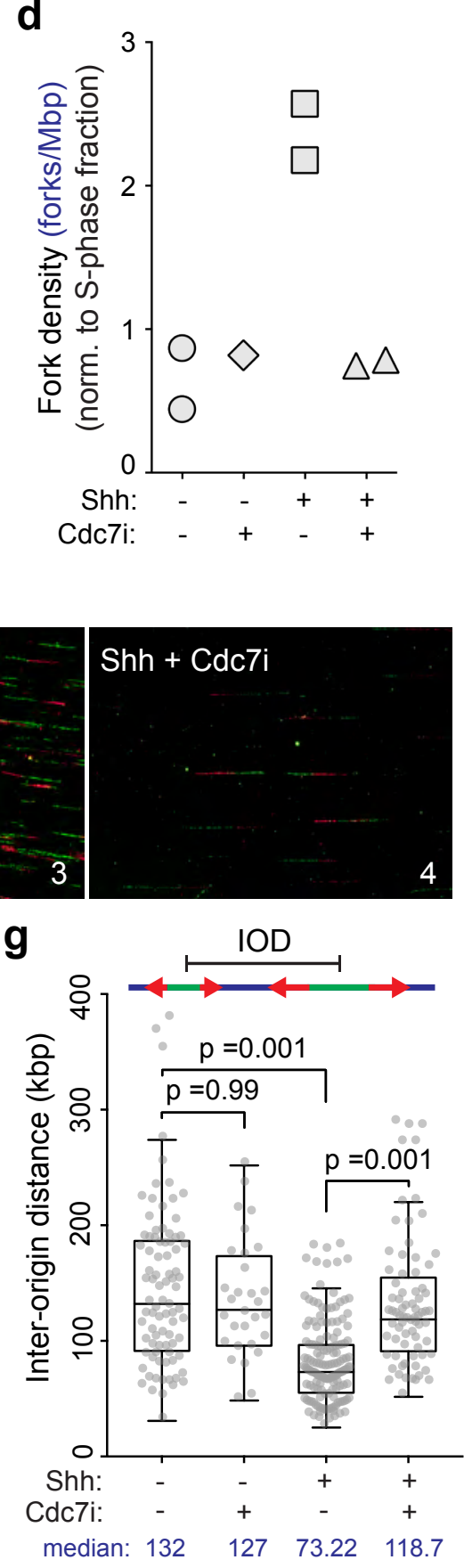
a

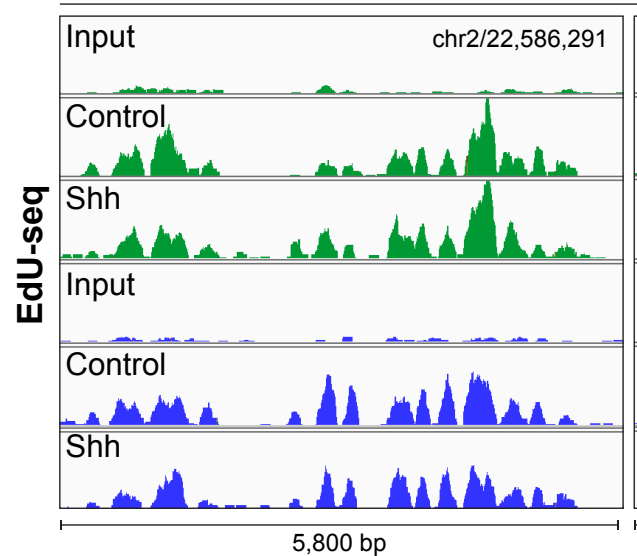

b

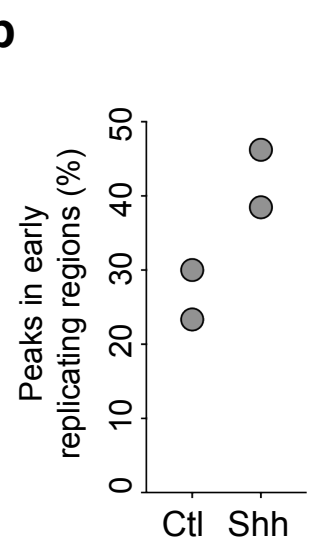

C

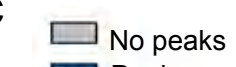

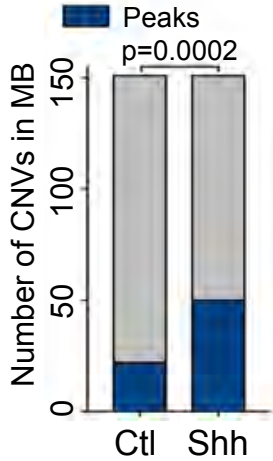

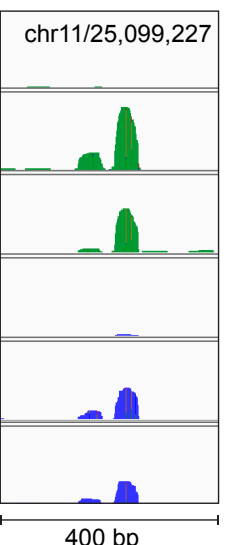

d
Shh-dependent initiation zones

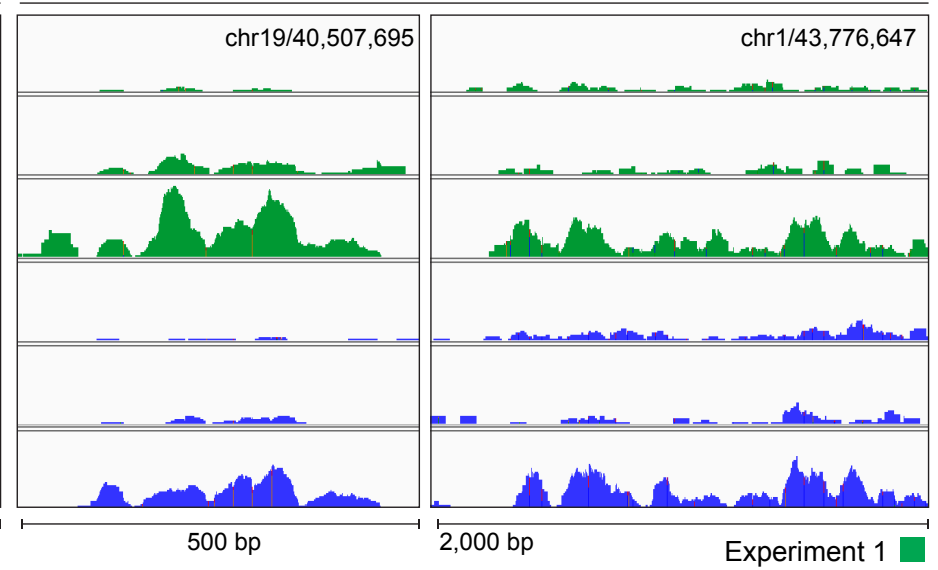

Experiment 2

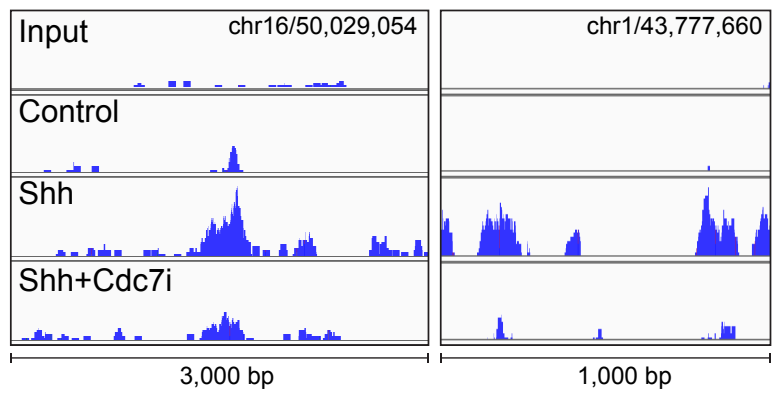


a

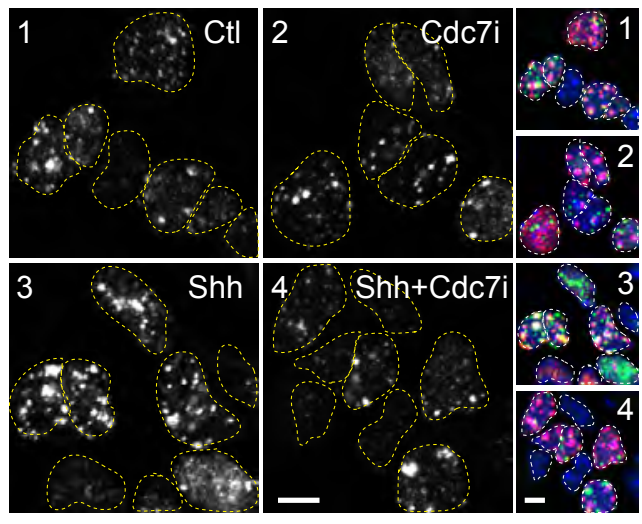

C

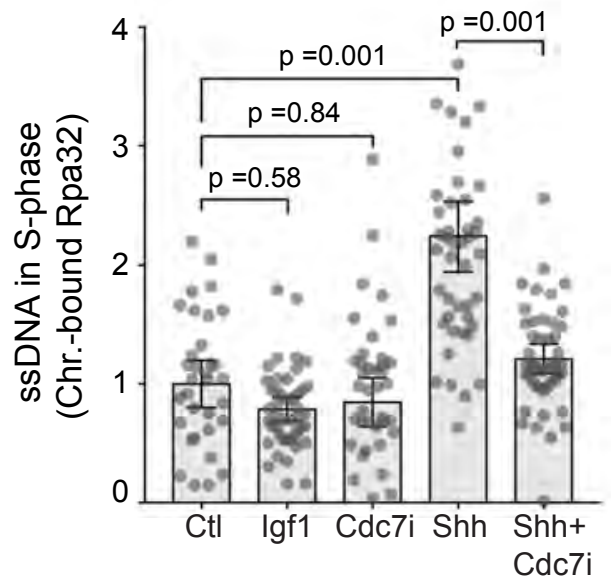

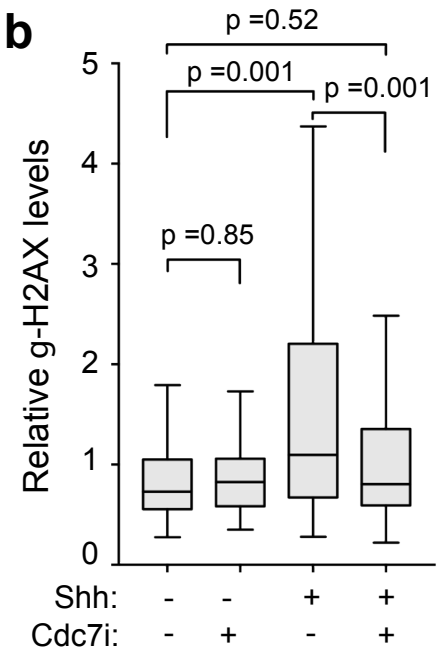

d

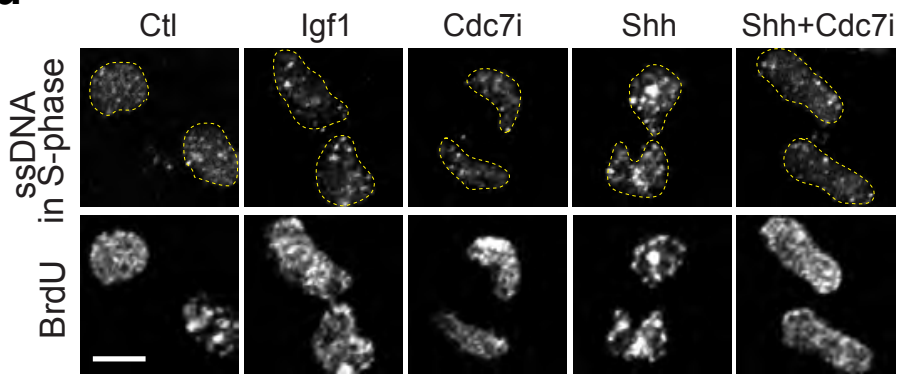

Ctl

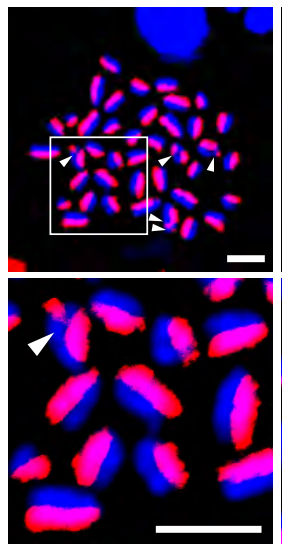

Igf1

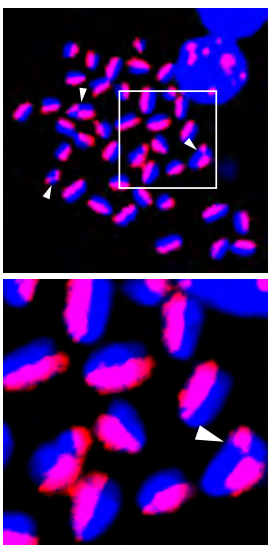

Shh

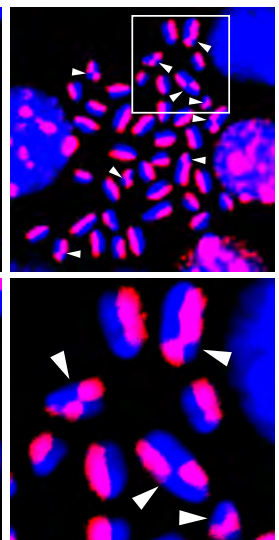

Shh+Cdc7i

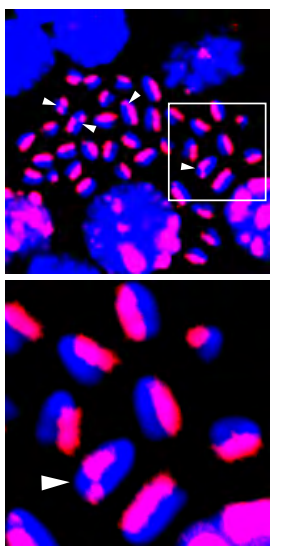


a Ptch $1^{+/-}$mice

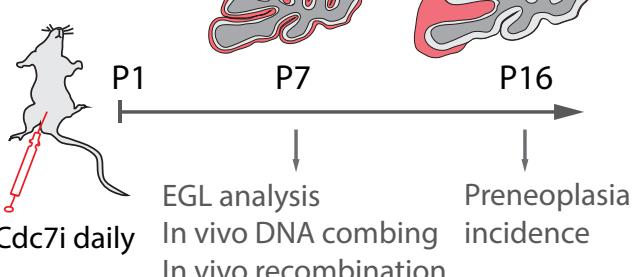
In vivo recombination

e

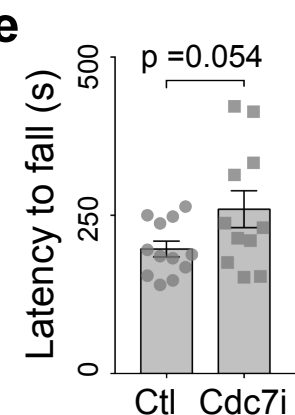

f

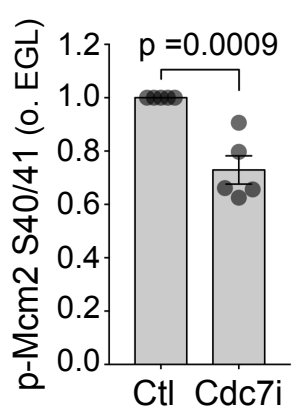

b

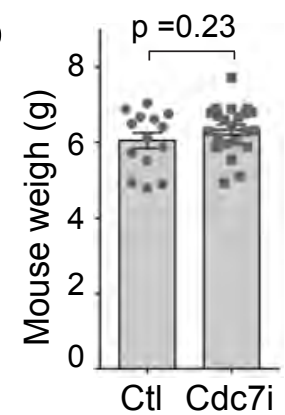

g

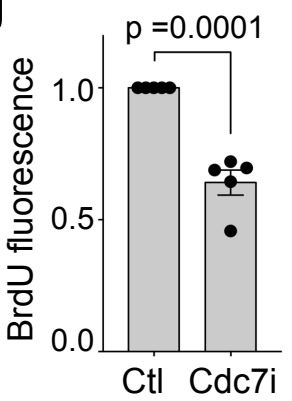

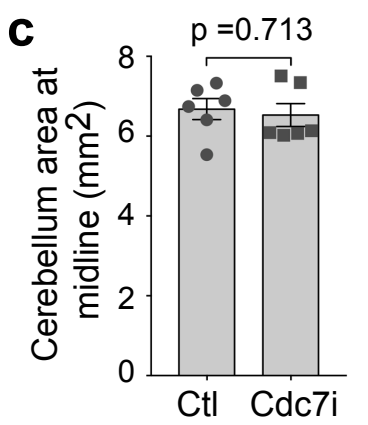

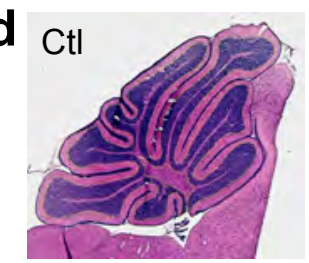

Cdc7i

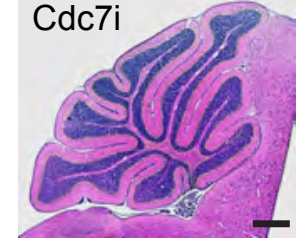

h
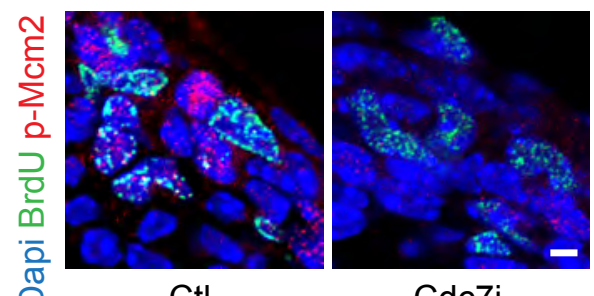

Cdc7i i

in vivo DNA combing

Ctl or Cdc7i CldU IdU

Wher

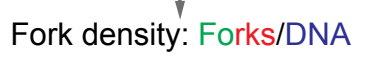
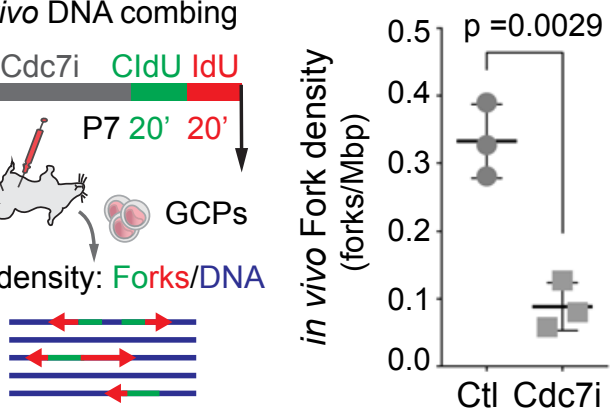

j
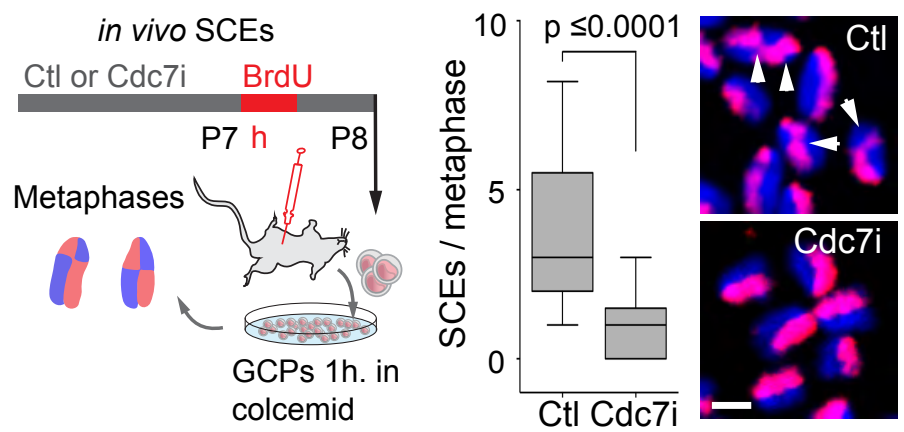

k

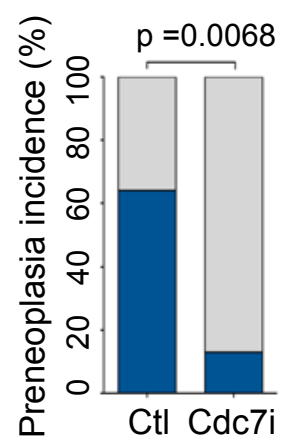

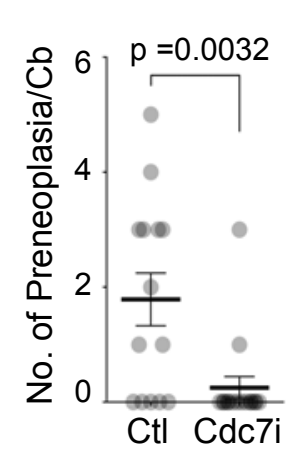

m

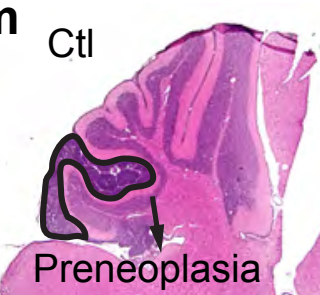

n

Shh-induced replication stress

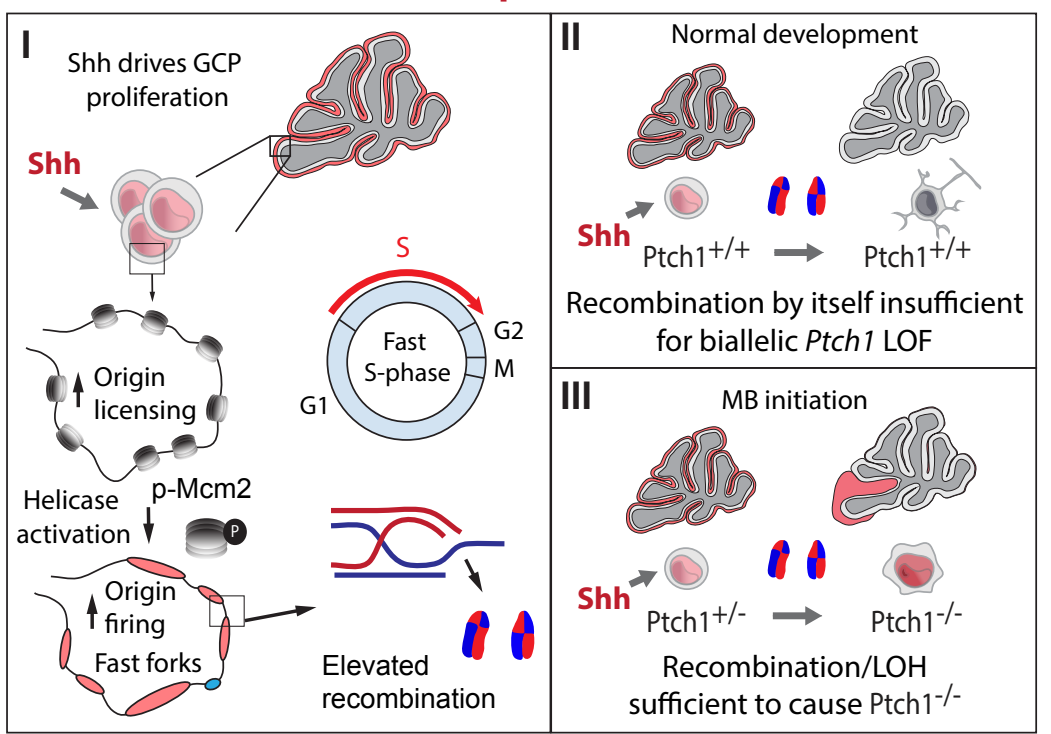



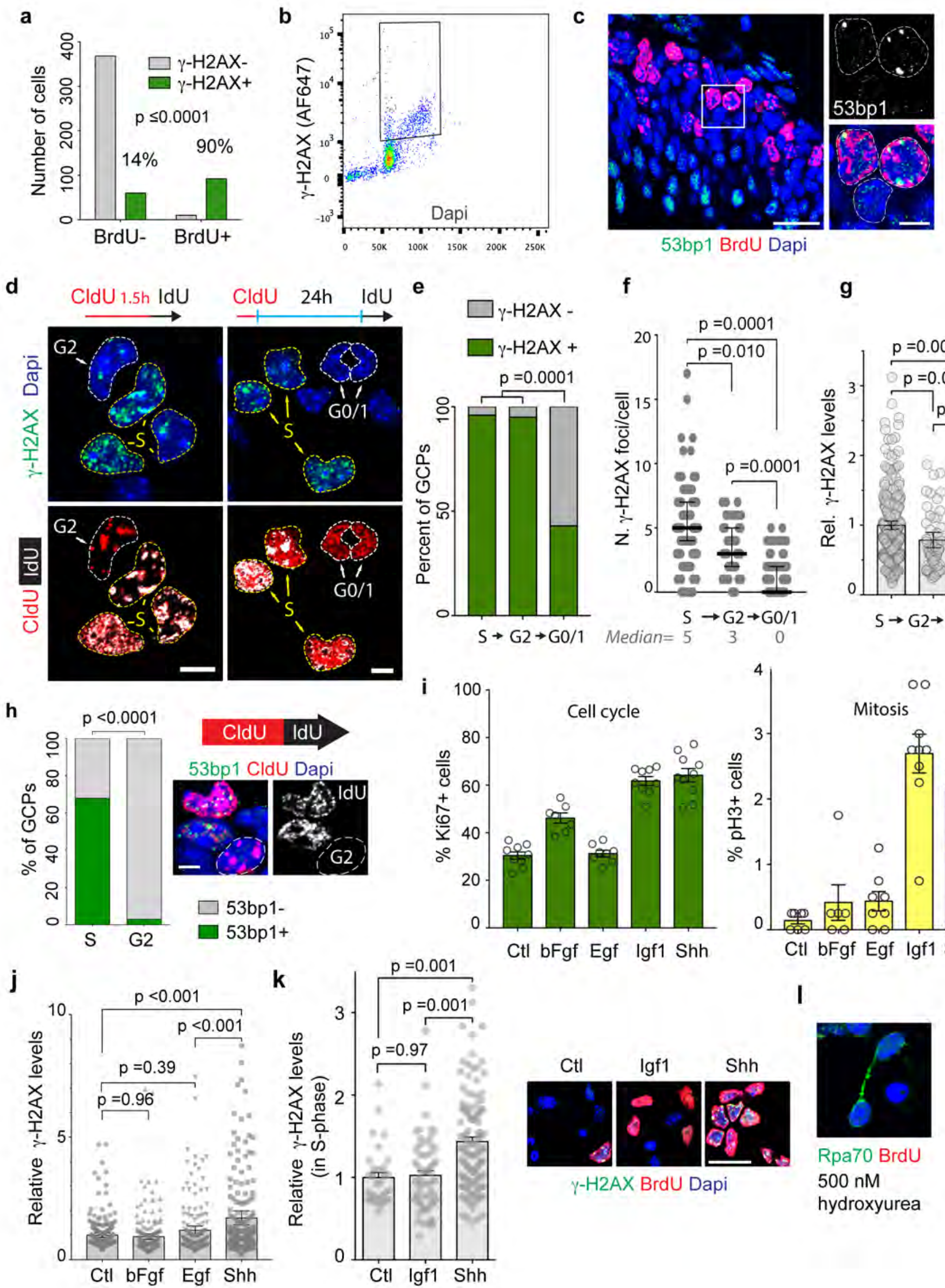

f

53bp1 BrdU Dapi

g
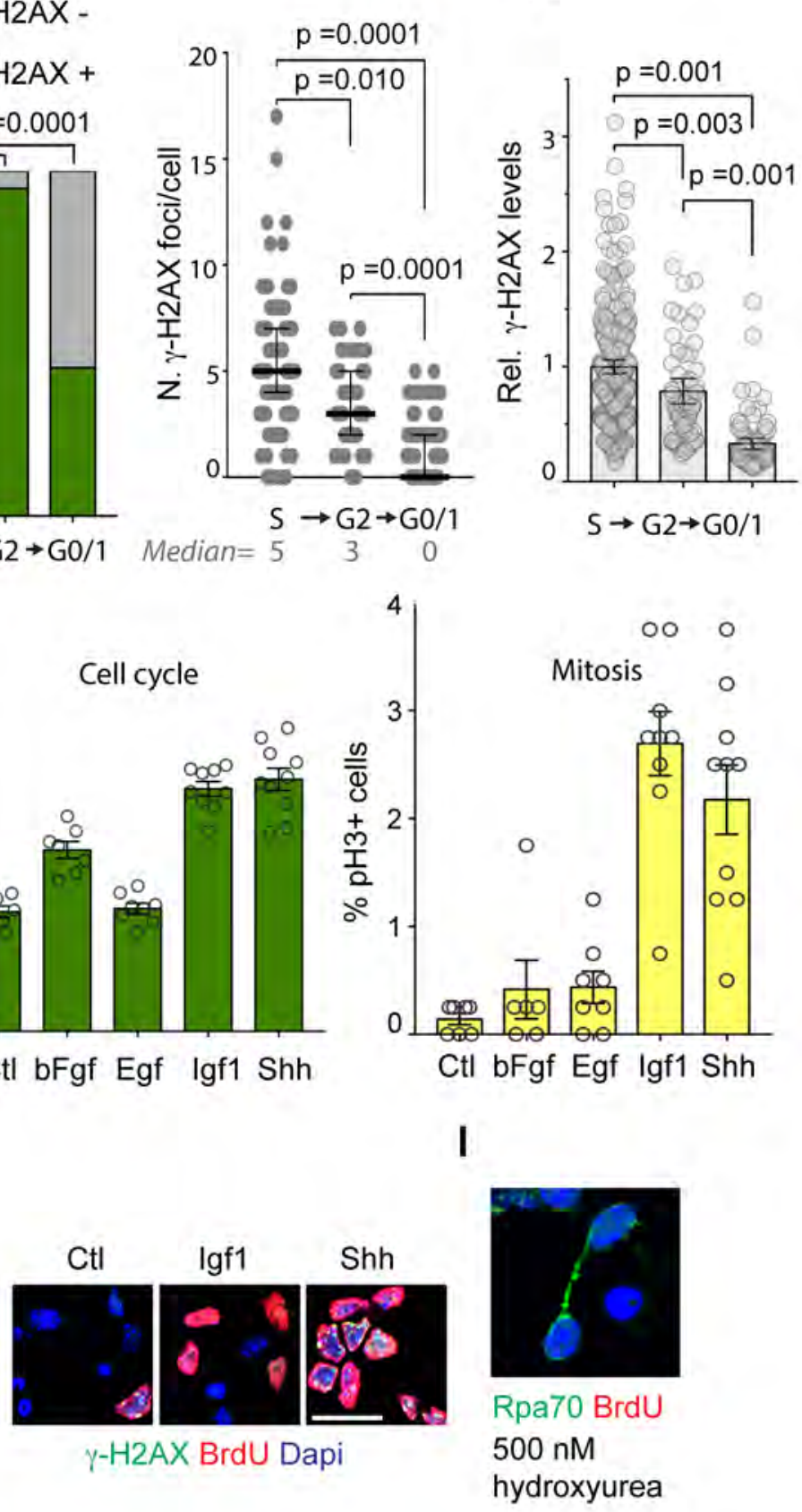
a Intestinal epithelium EGL

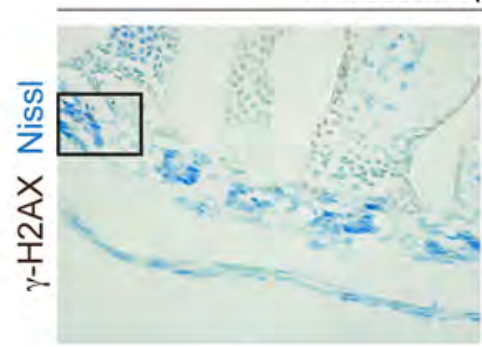

b
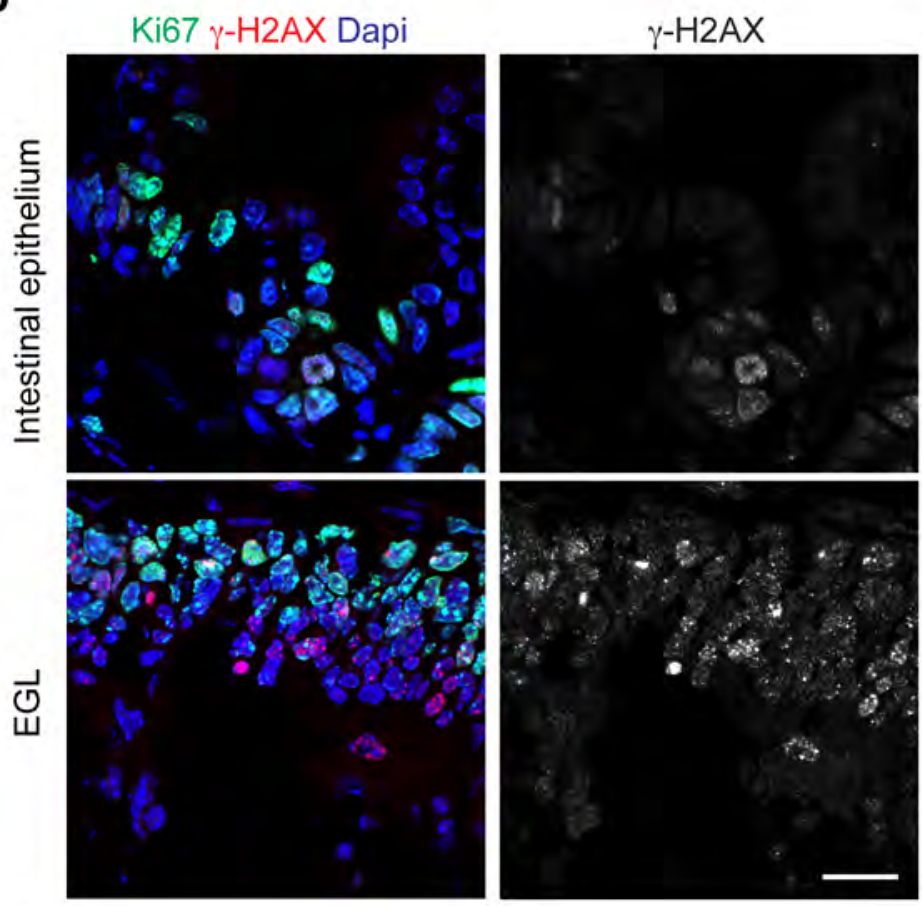

c

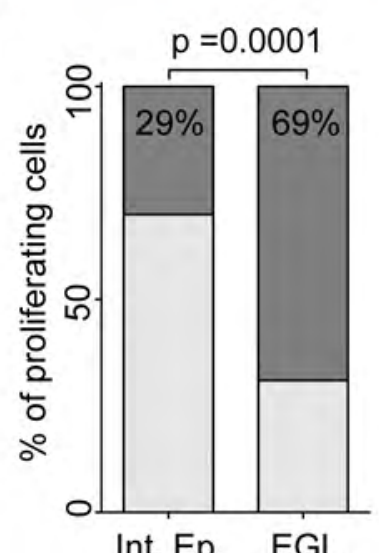

Int. Ep. EGL d

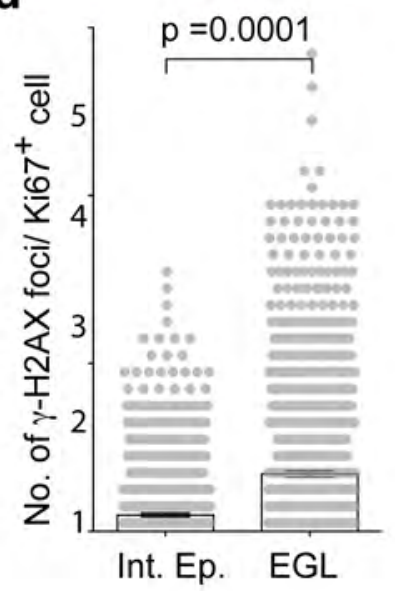

e

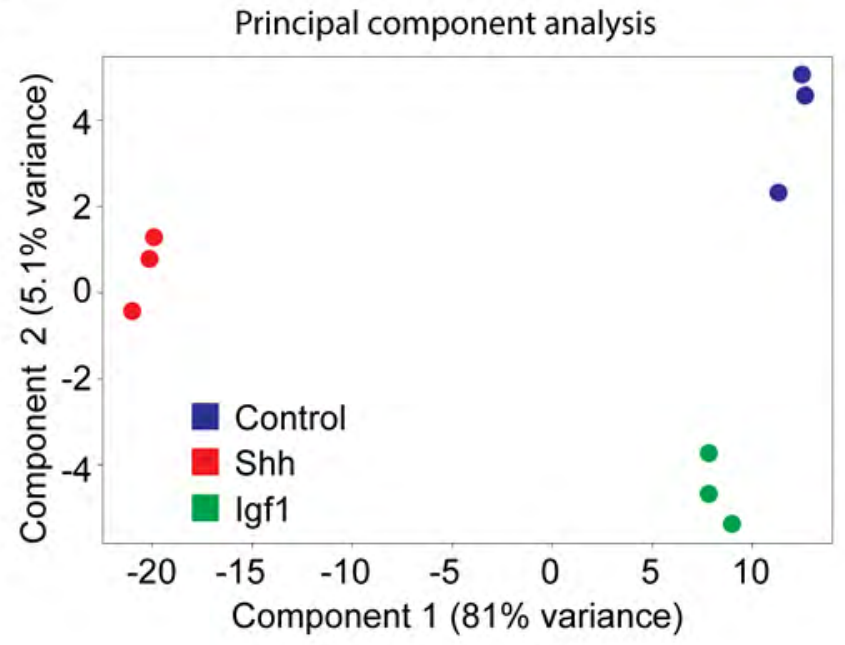

f
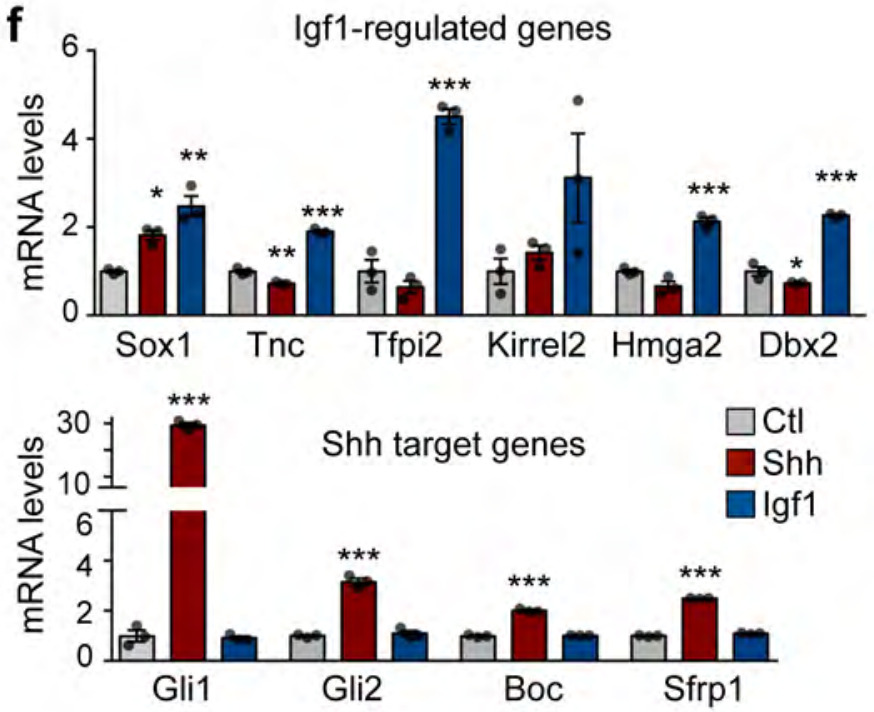


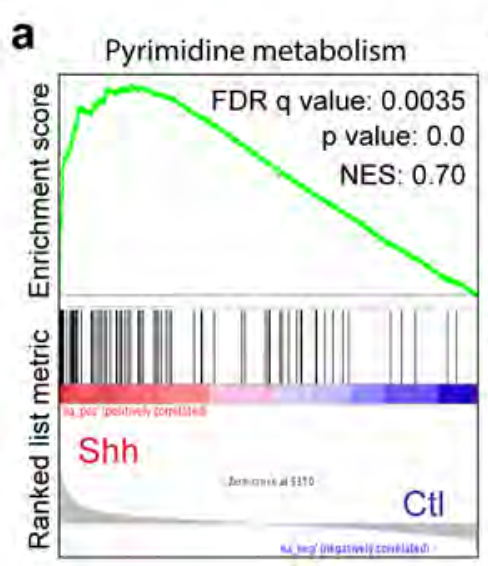

b
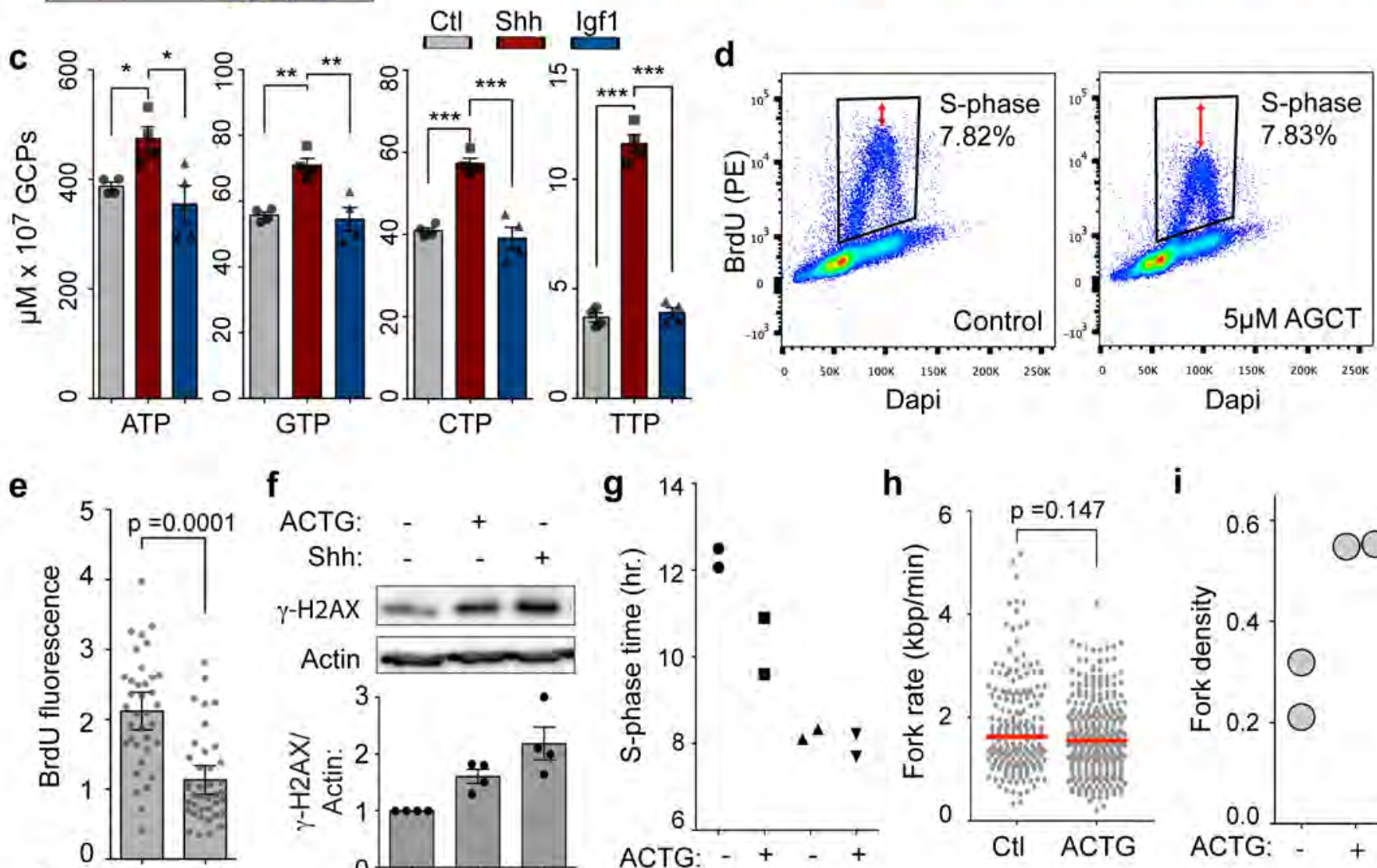

AGCT: - +
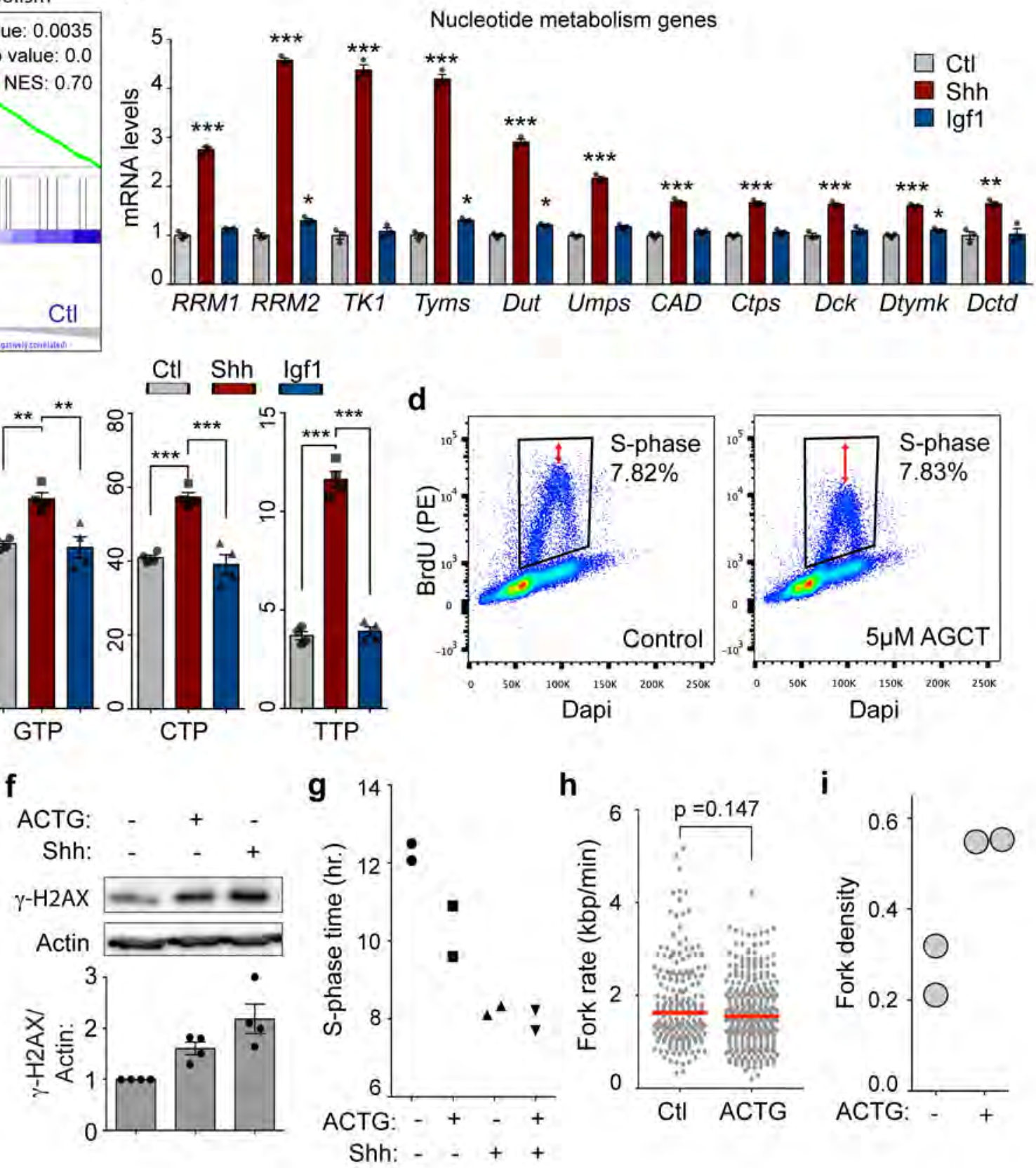

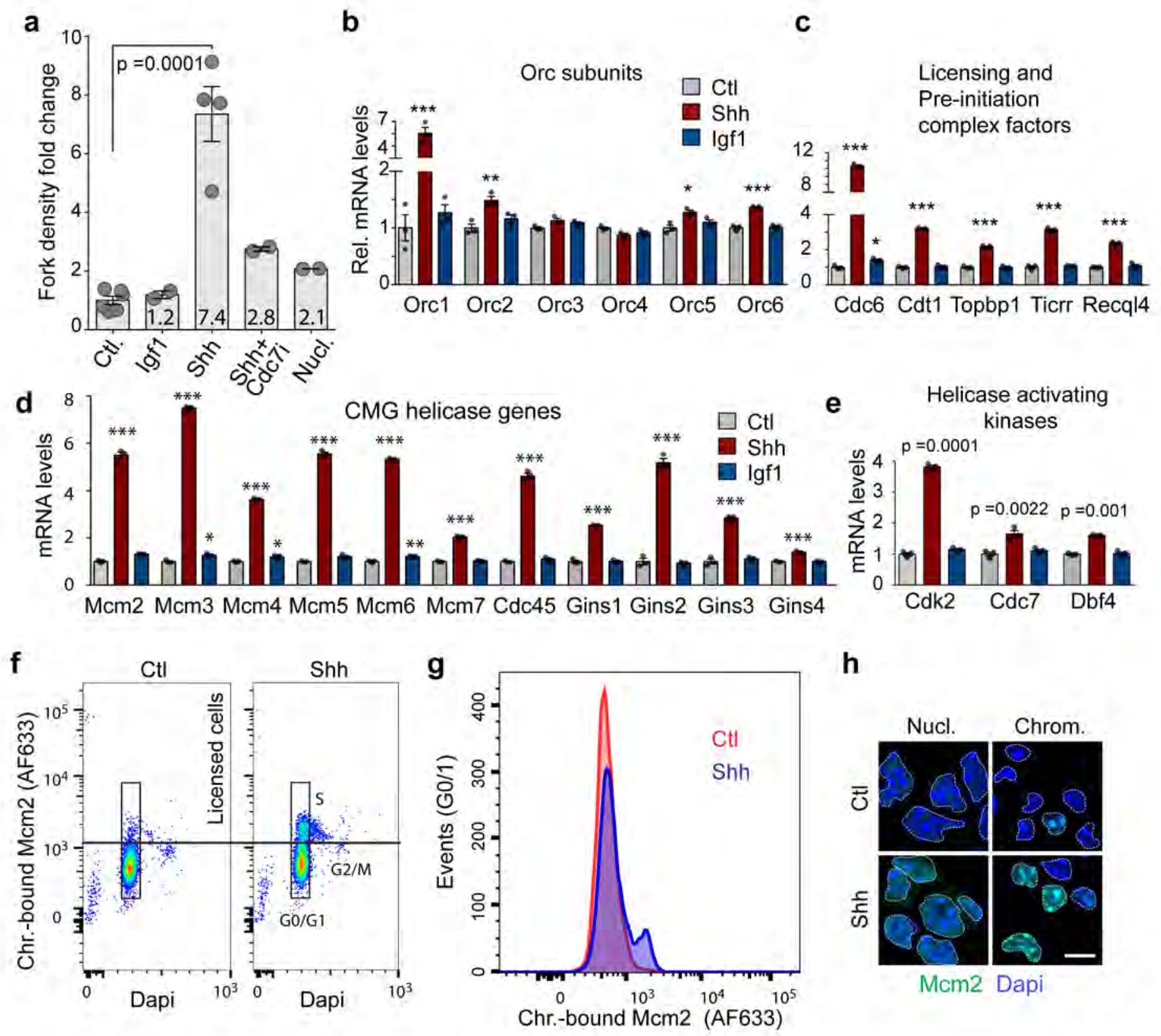

h

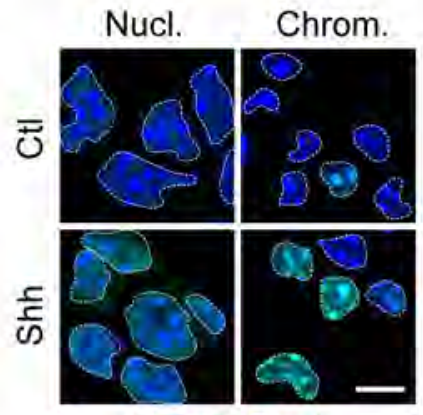

Mcm2 Dapi 

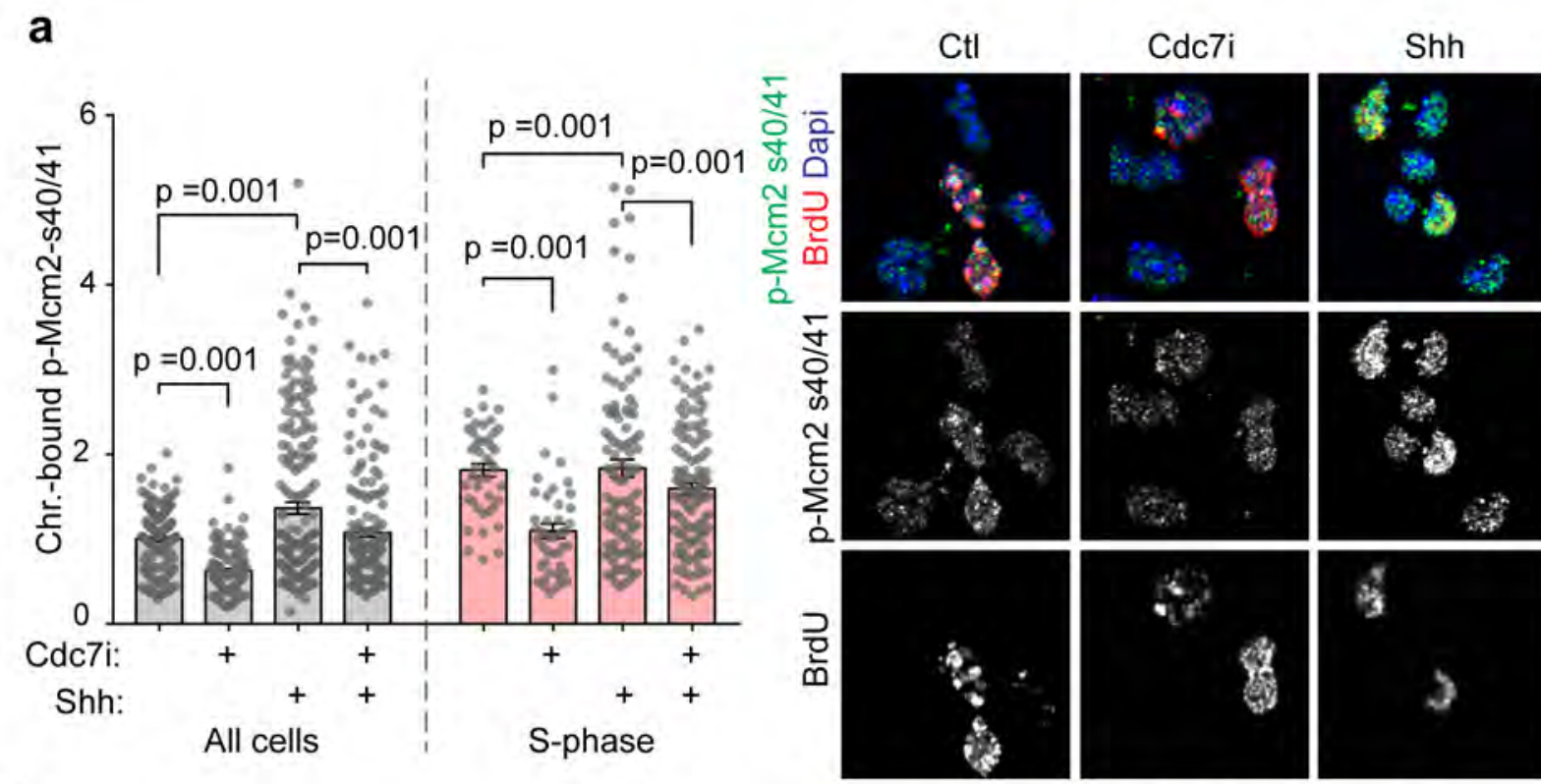

$\mathrm{Shh}+\mathrm{Cdc7i}$

b
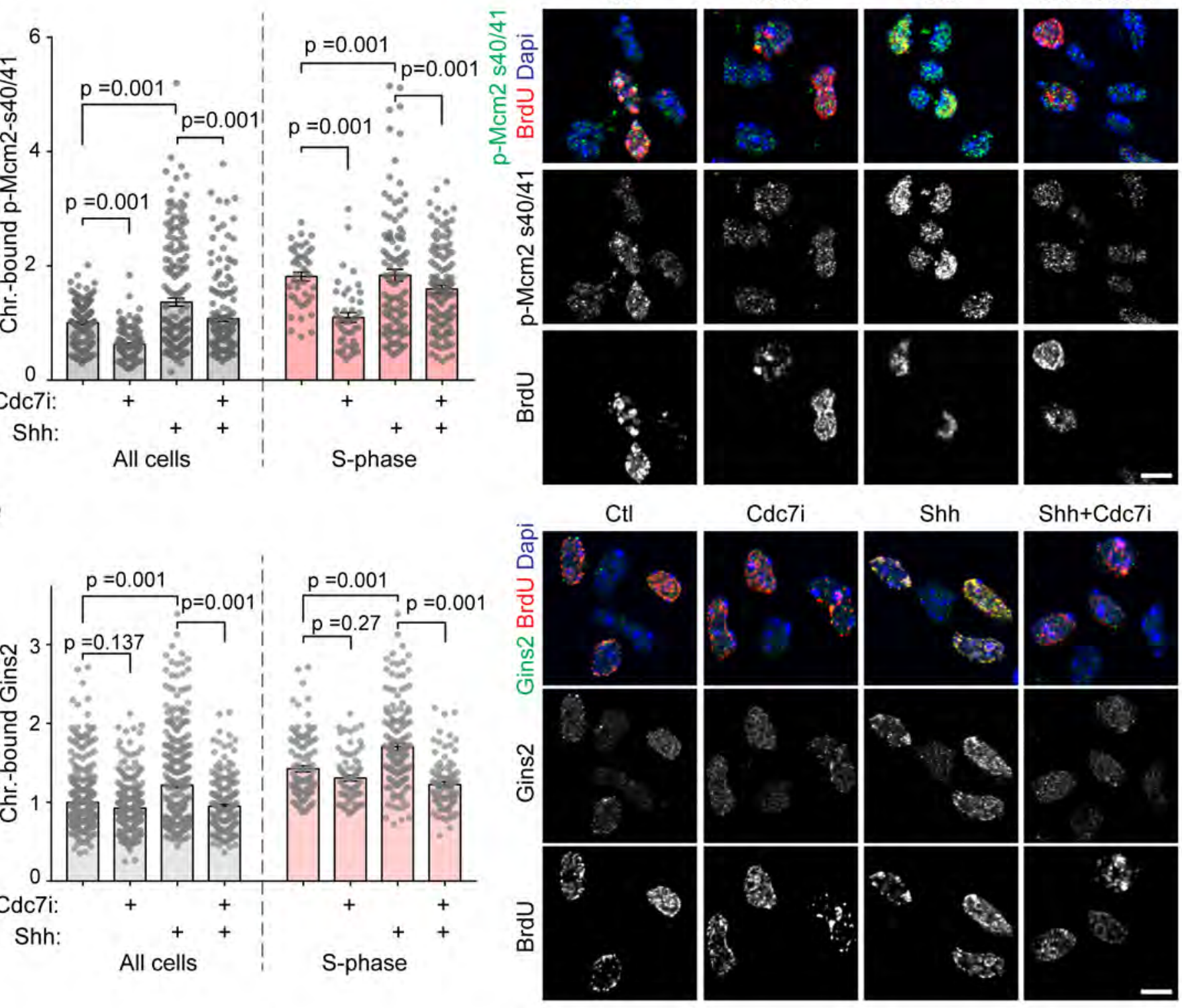

Shh+Cdc7i

C

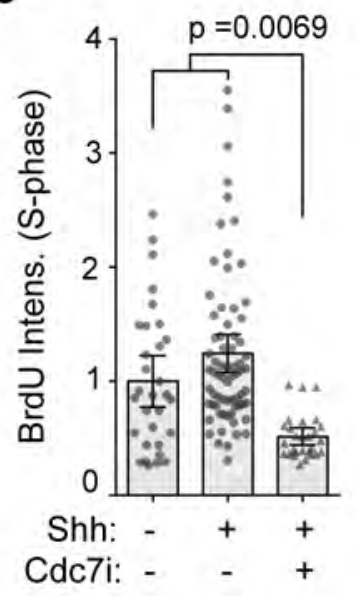

d

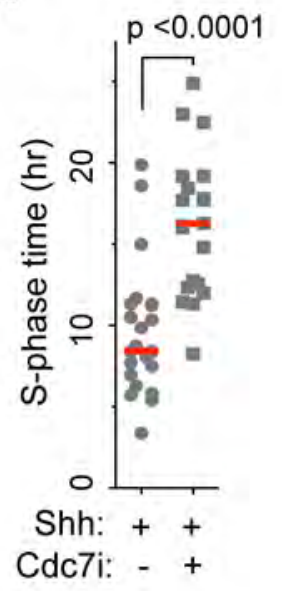



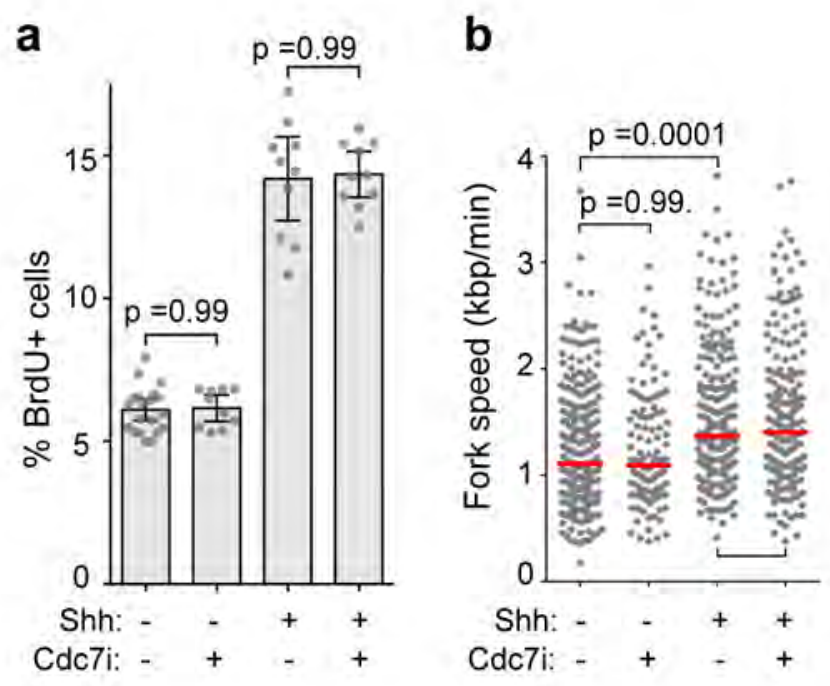

d
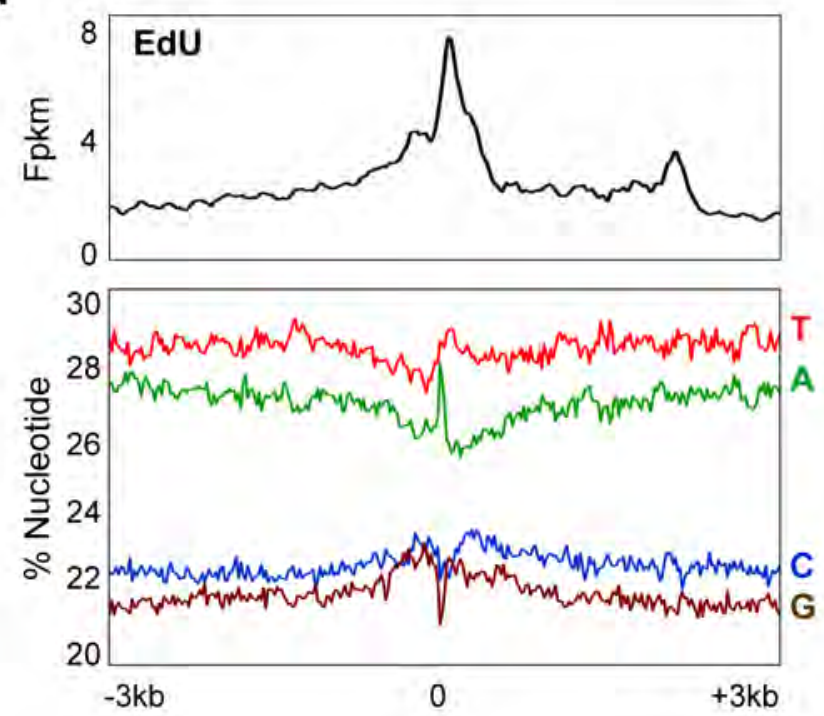

f

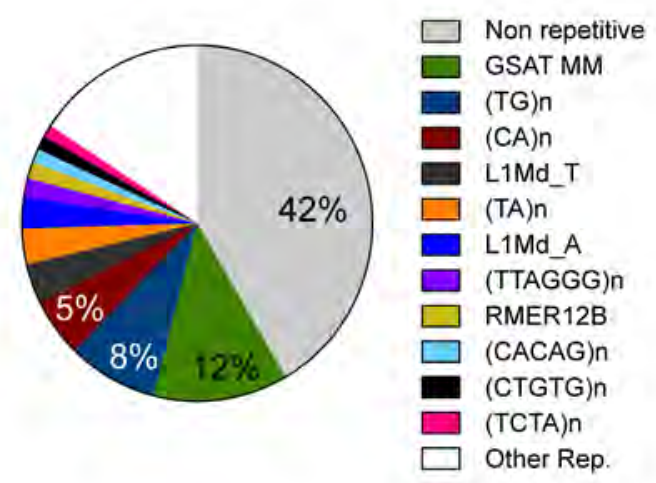

C

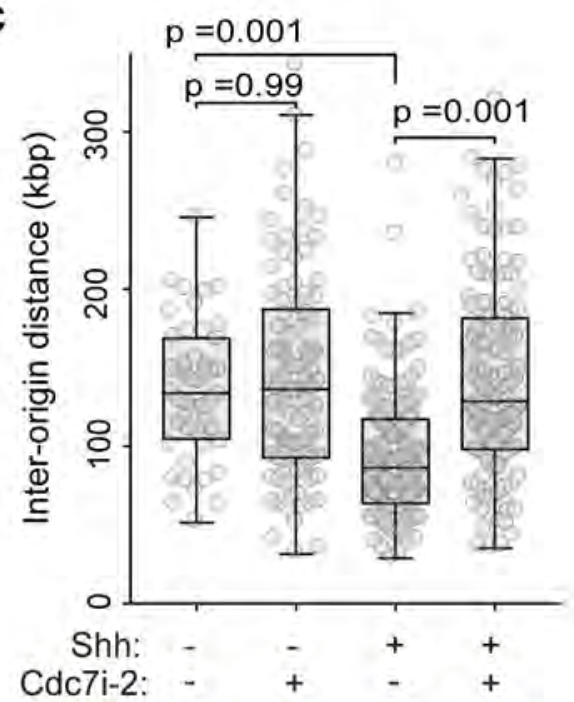

median: $134.0 \quad 136.4 \quad 86.5 \quad 128.6$

e

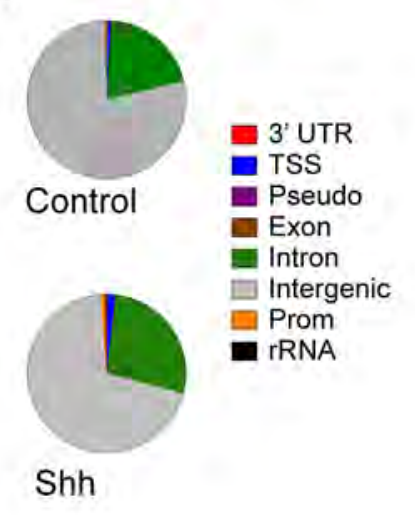

g

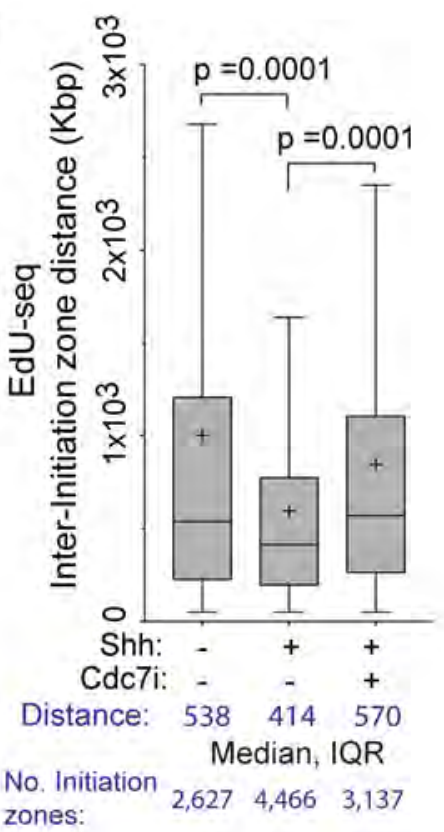



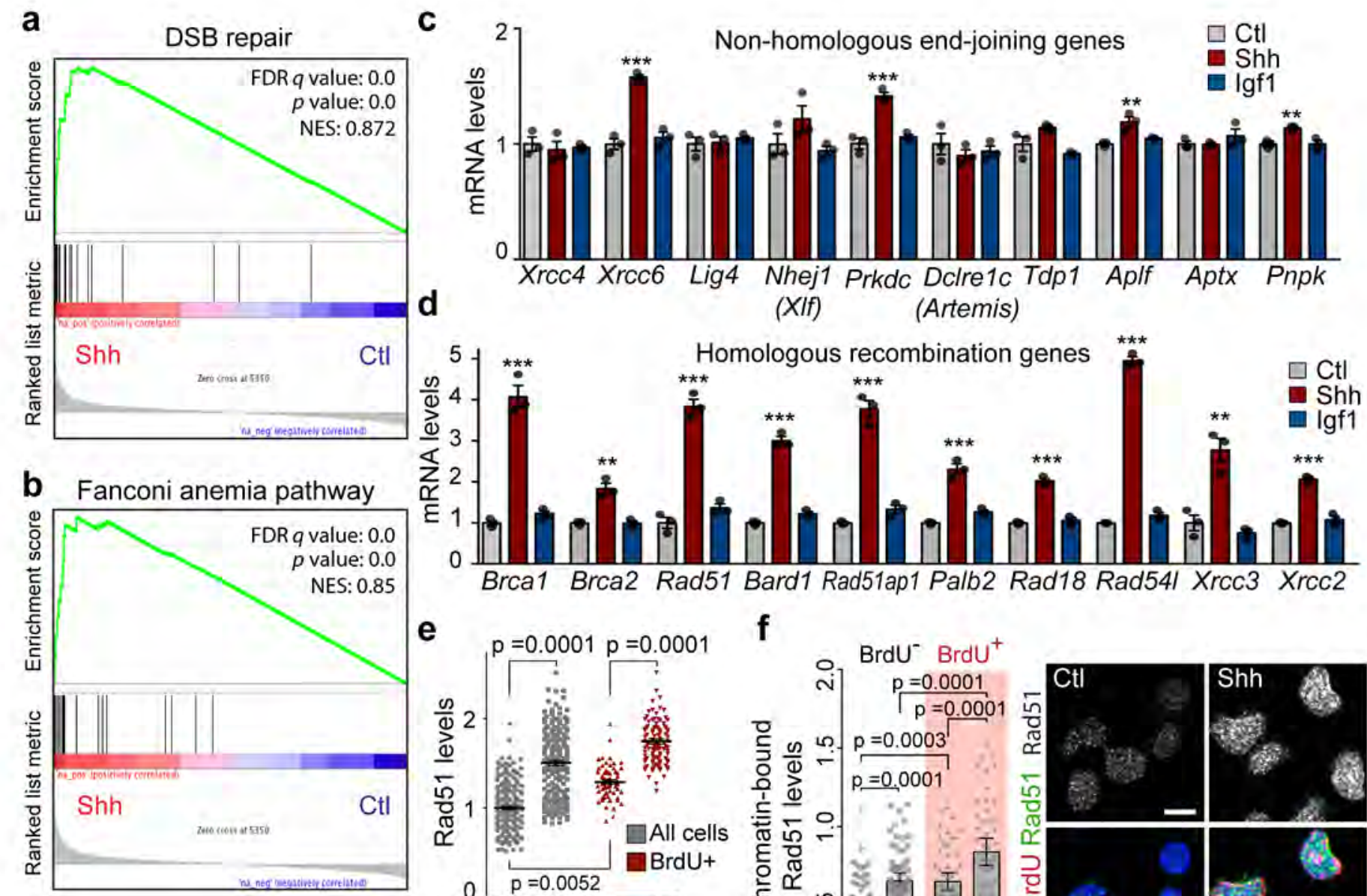

g

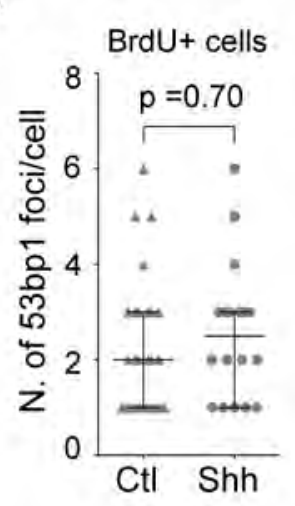

h

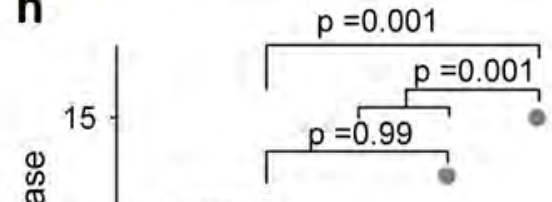

Shh:

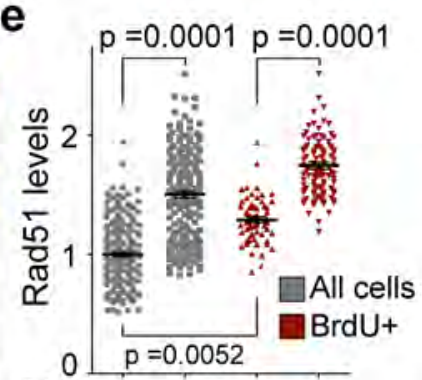

f
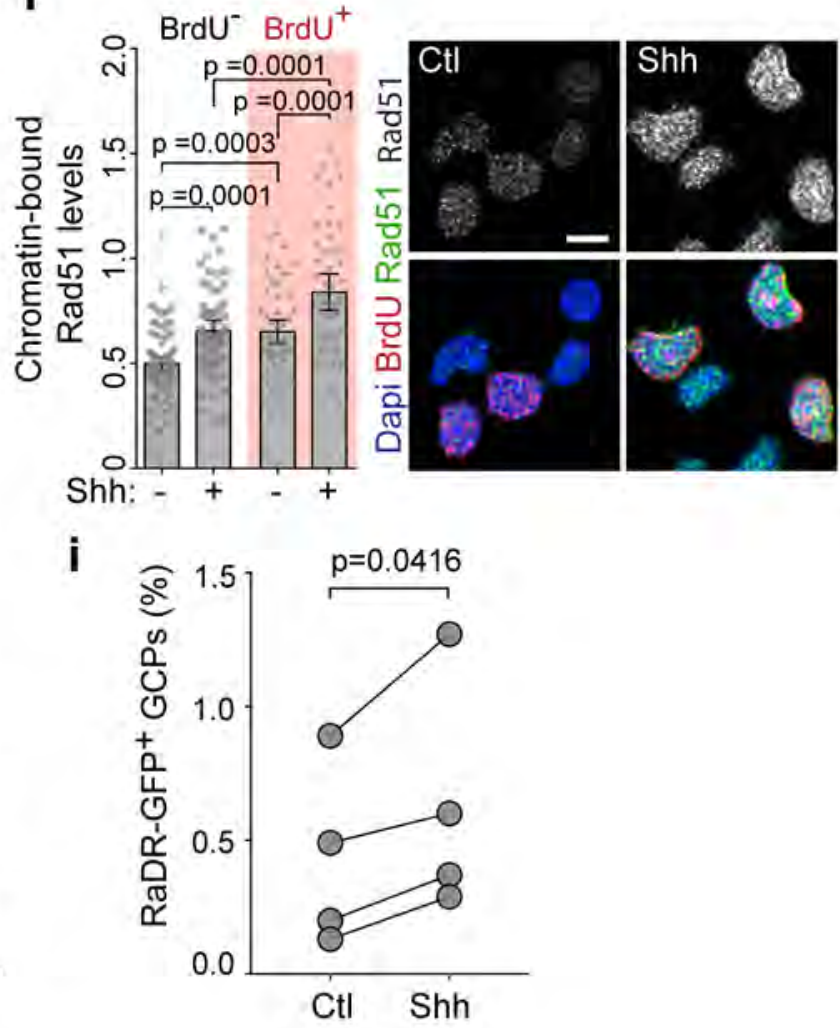

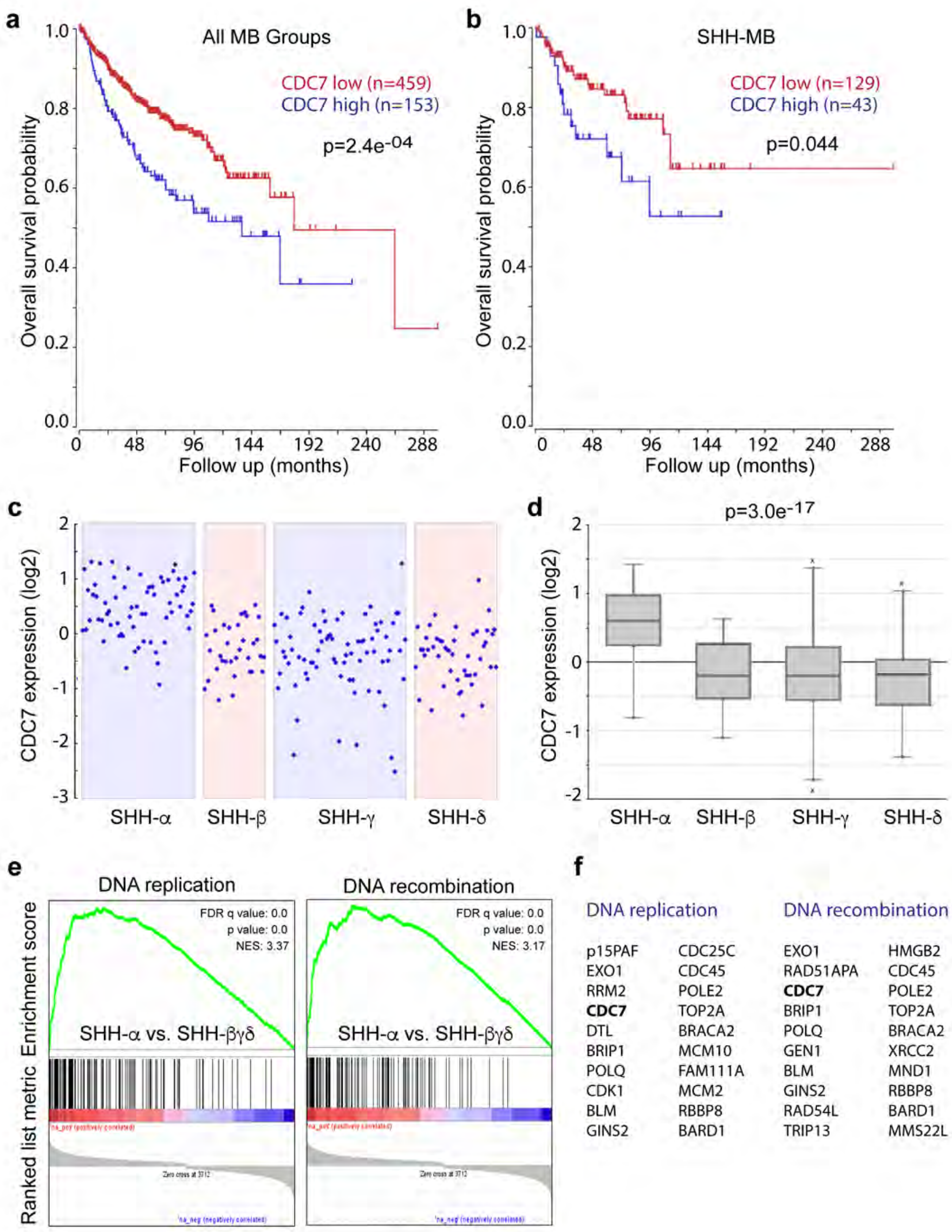

DNA replication DNA recombination

$\begin{array}{llll}\text { P15PAF } & \text { CDC25C } & \text { EXO1 } & \text { HMGB2 } \\ \text { EXO1 } & \text { CDC45 } & \text { RAD51APA } & \text { CDC45 } \\ \text { RRM2 } & \text { POLE2 } & \text { CDC7 } & \text { POLE2 } \\ \text { CDC7 } & \text { TOP2A } & \text { BRIP1 } & \text { TOP2A } \\ \text { DTL } & \text { BRACA2 } & \text { POLQ } & \text { BRACA2 } \\ \text { BRIP1 } & \text { MCM10 } & \text { GEN1 } & \text { XRCC2 } \\ \text { POLQ } & \text { FAM111A } & \text { BLM } & \text { MND1 } \\ \text { CDK1 } & \text { MCM2 } & \text { GINS2 } & \text { RBBP8 } \\ \text { BLM } & \text { RBBP8 } & \text { RAD54L } & \text { BARD1 } \\ \text { GINS2 } & \text { BARD1 } & \text { TRIP13 } & \text { MMS22L }\end{array}$


a Normalized enrichment score (Proteomics)

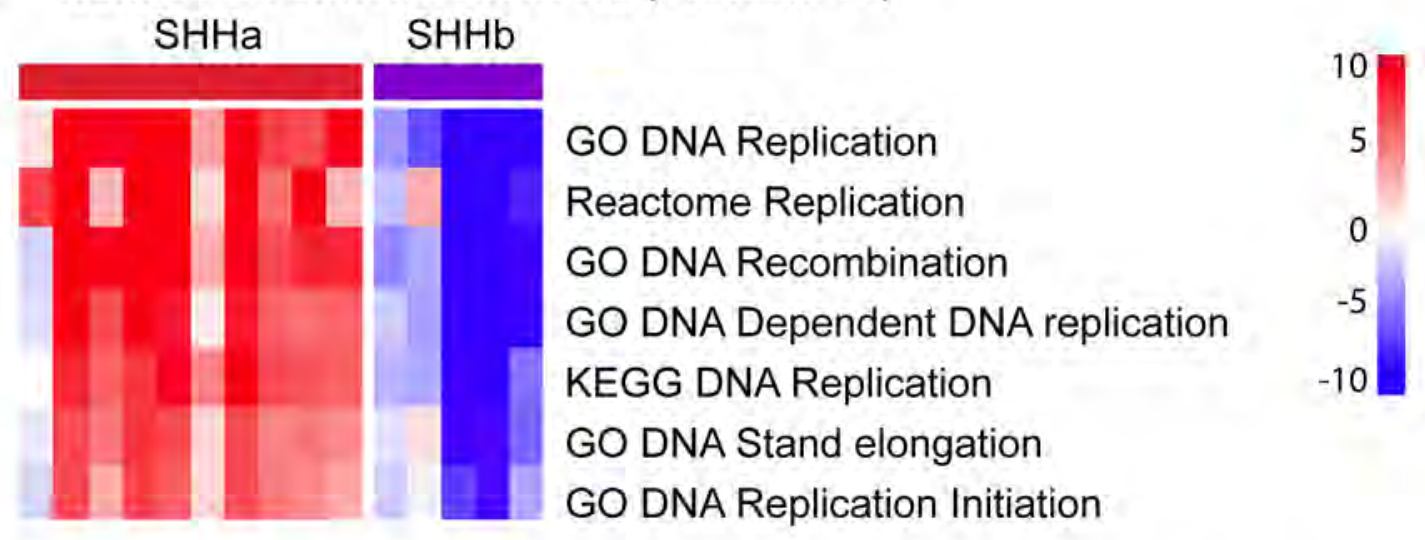

b

GO DNA Recombination mRNA

Protein
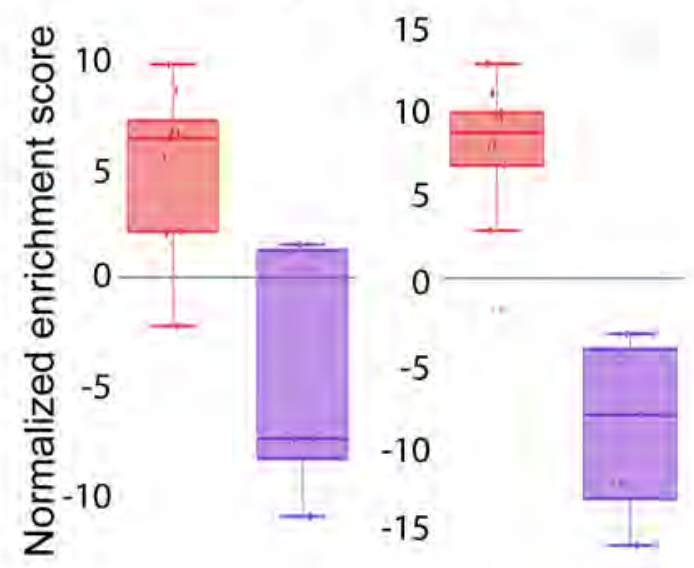

c MCM2 MRNA

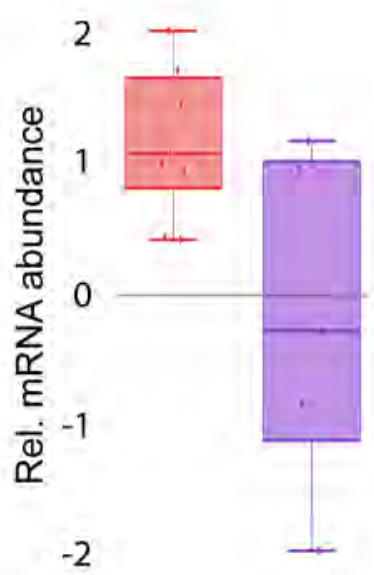

d

MCM2 Protein

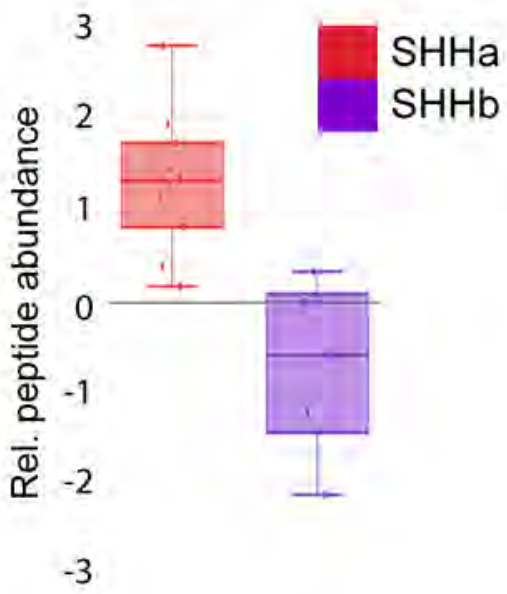

e MCM2 p-Ser40
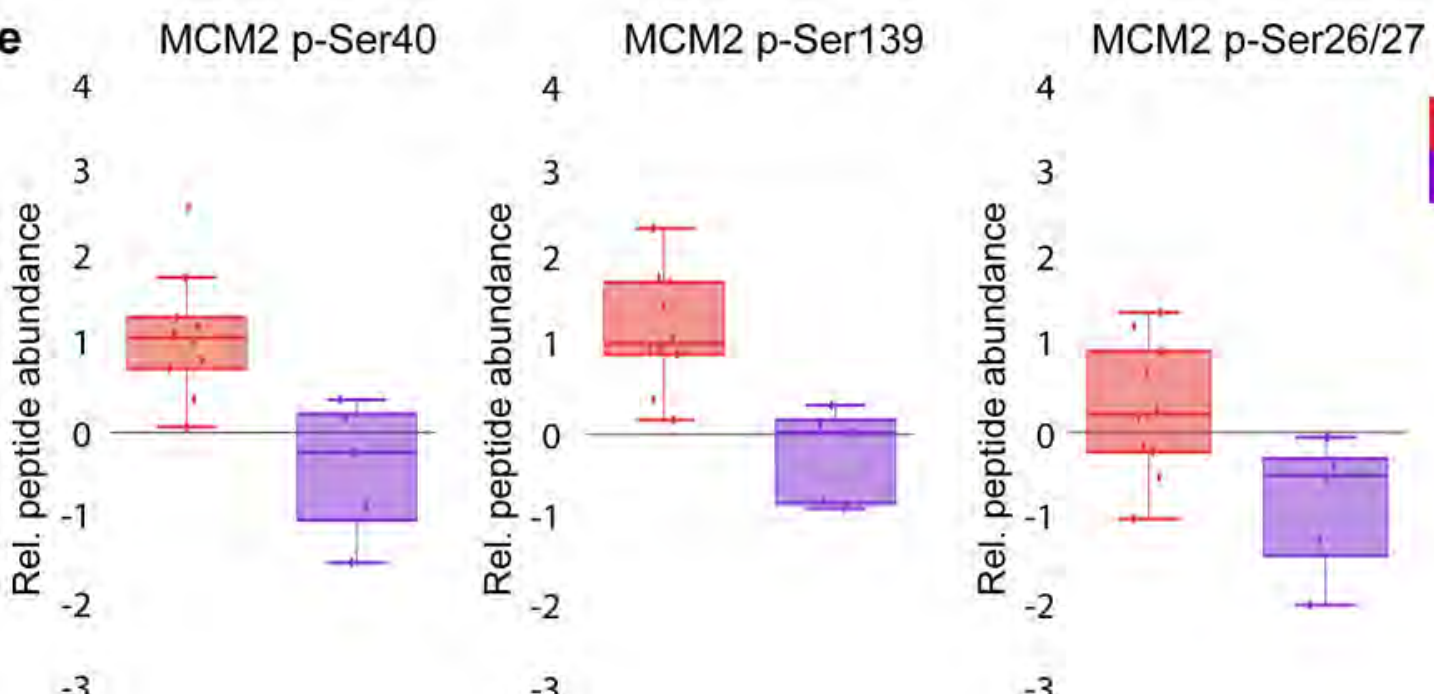

$\mathrm{SHHa}$

$\mathrm{SHHb}$ 


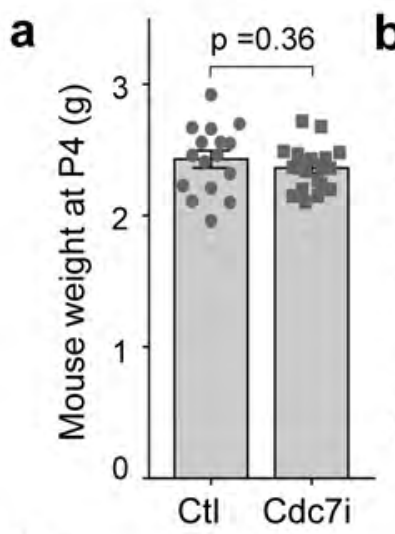

e

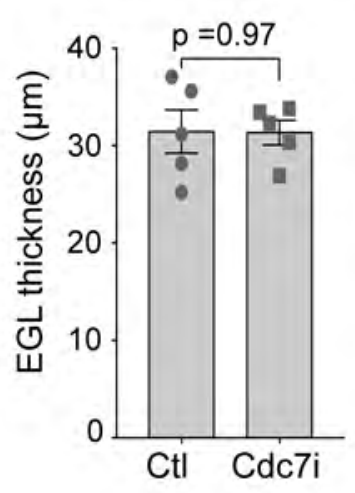

h

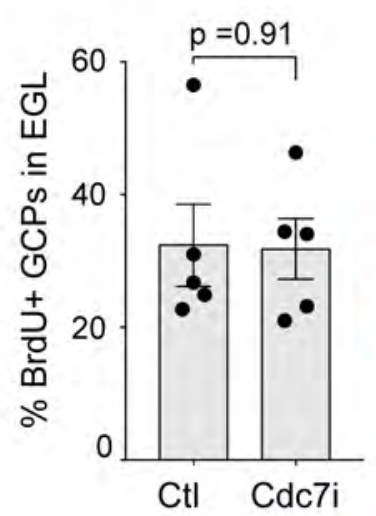

k
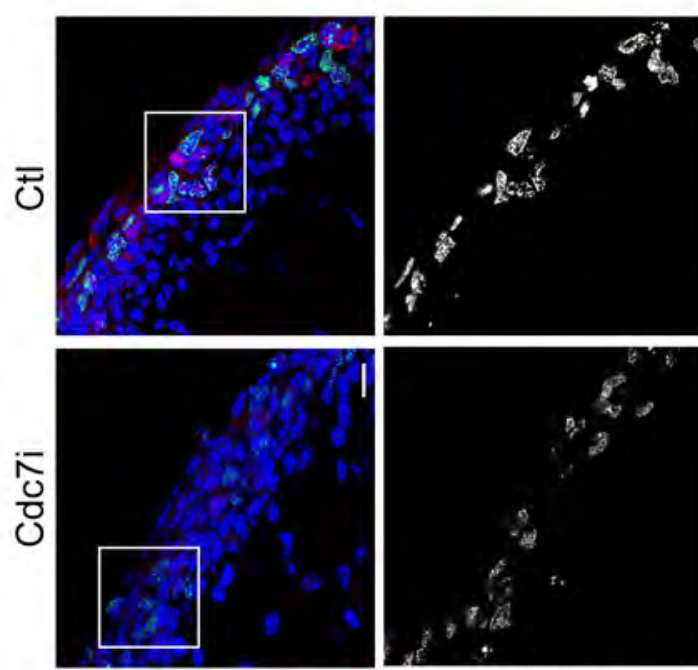

Dapi BrdU p-Mcm2

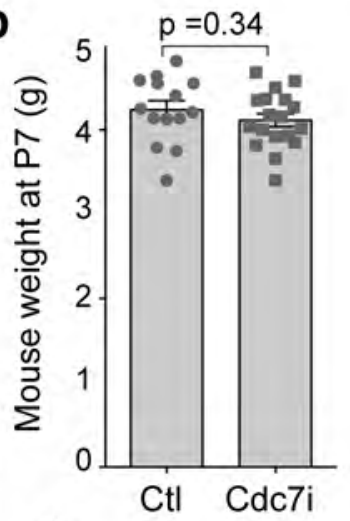

f
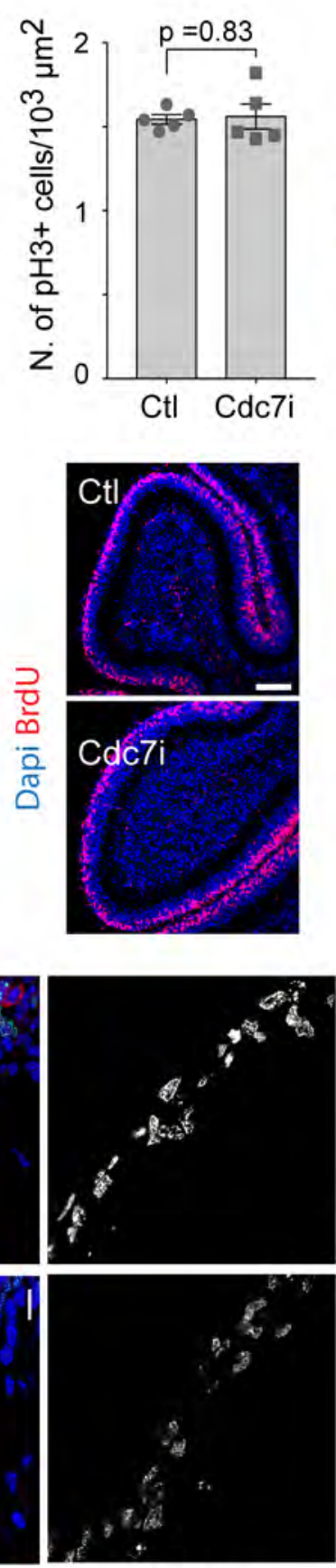

BrdU
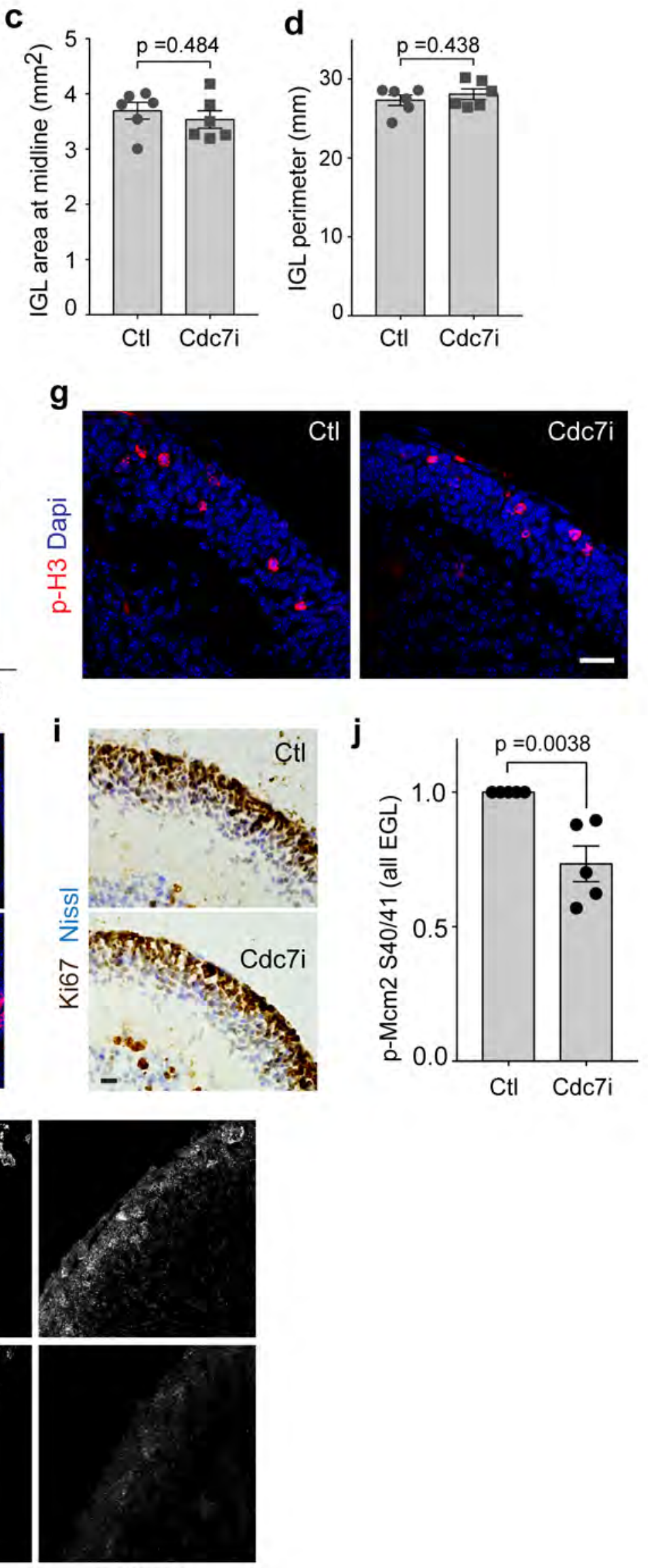

p-Mcm2 